FAMILY AWAY FROM HOME: SUPPORT SYSTEMS FOR MULTIGENERATIONAL HISPANIC STUDENTS AT A HISPANIC SERVING INSTITUTION (HSI)

\author{
As A Dissertation submitted to the faculty of \\ 35 \\ 2016 \\ EDD \\ $\cdot M 87$ \\ San Francisco State University \\ In partial fulfillment of \\ the requirements for \\ the Degree \\ Doctor of Education \\ In \\ Educational Leadership
}

by

Omar R. Murillo

San Francisco, California

January 2016 
Copyright by

Omar R. Murillo

2016 


\section{CERTIFICATION OF APPROVAL}

I certify that I have read Family Away from Home: Support Systems for Multi-Generational Hispanic Students at a Hispanic Serving Institution by Omar R. Murillo, and that in my opinion this work meets the criteria for approving a disseration submitted in partial fulfillment of the requirement for the degree Doctorate in Education: Educational Leadership at San Francisco State University.

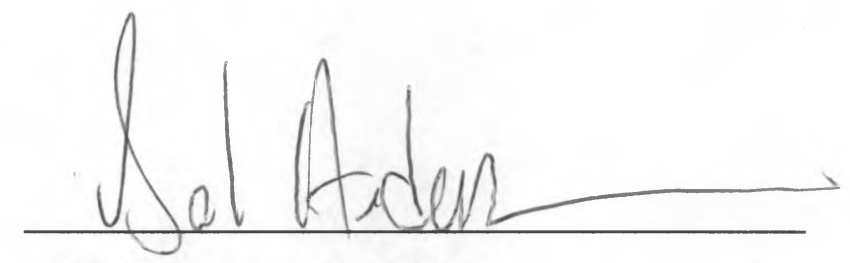

Barbara Henderson, Ph.D.

Professor
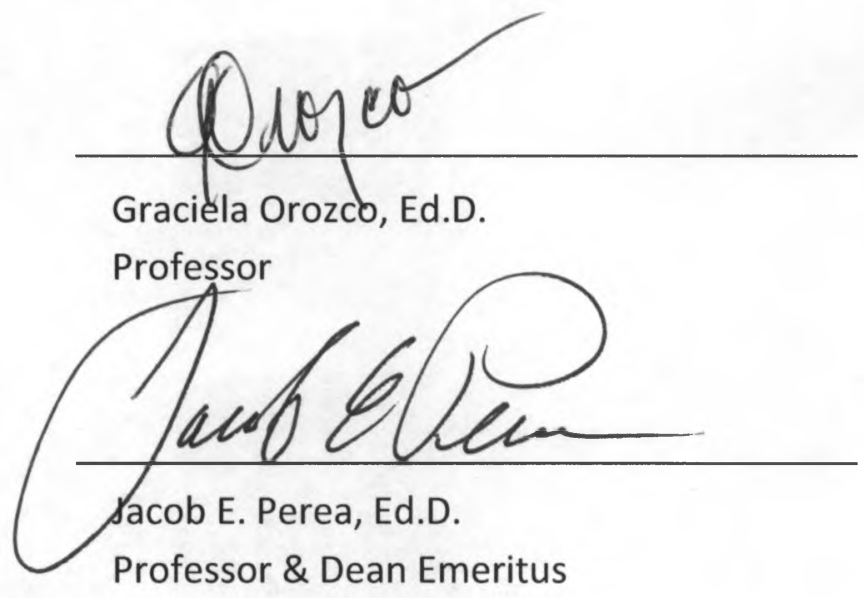


\title{
FAMILY AWAY FROM HOME: SUPPORT SYSTEMS FOR MULTI- GENERATIONAL HISPANIC STUDENTS AT A HISPANIC SERVING INSTITUTION (HSI)
}

\author{
Omar R. Murillo \\ San Francisco, California \\ 2016
}

This dissertation is a qualitative study based on data collected from in-depth interviews with Raza students currently attending Central Coast University (CCU), a Hispanic Serving Institution (HSI) in California. The study examines the role of pre-college life and in-college experiences on Raza students' perceptions of educational support. The focus was to examine the supports that multi-generational Raza students across genders perceive as effective. The 25 interview participants were selected through purposive sampling to represent the diversity of Raza students at the institution. Data were analyzed through multiple cycles of coding, which led to the development of a Hispanic College Student Support Model, the study's conceptual framework. Overall findings demonstrate that the educational experience, home environment, and family influence (which includes the role of gender and generational status), have intersected to create different levels of oppression, sense of belonging, and family environment. Specifically, the study finds that the third generational students shared the most positive experiences about support. Additionally, females were more likely to discuss the role of family and the value of interactions, while males were more likely to describe how they independently navigated obstacles they faced in education. Implications and recommendations will contribute the development of the Campus Diversity Plan at CCU to help the institution be more inclusive, such that it positively impacts students' academic success and personal growth.

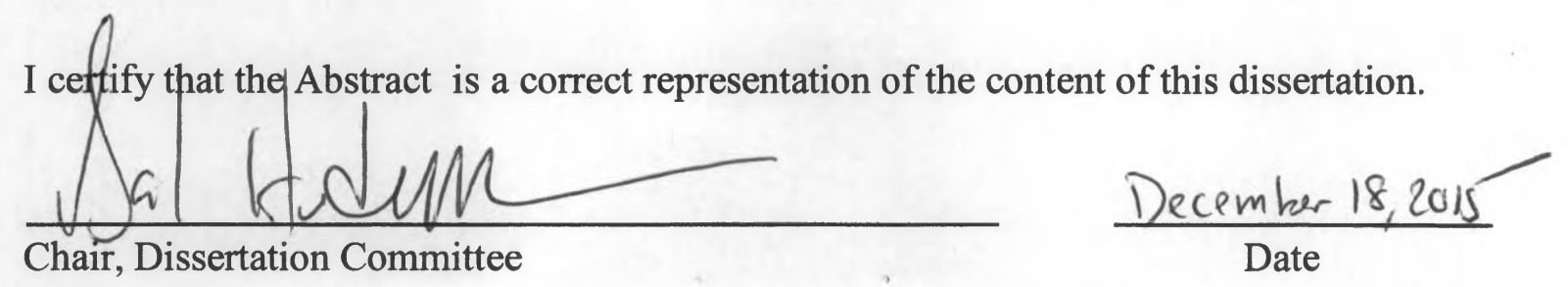




\section{ACKNOWLEDGEMENTS}

I first want to thank my amazing parents Martha and Refugio Murillo. Thank you for your love, for believing in me, and for teaching me the value of an education. To my siblings - your energy, jokes, and support have brought so much joy to my life. Follow your dreams and know anything is possible. To my Primos/Primas - I love you all. Thank you for helping me de-stress throughout this whole process. Let's challenge each other to be better people for ourselves, our families, and our communities. To my Tias/Tios/Ama - Los quiero mucho! Gracias por su apoyo. Esta tesis es para Apa y Cuca! Ireliz - I wouldn't be here without your support. You helped me believe in myself. All love for you and Naz all day. Neri - You have been a blessing over the last few months. THANK YOU from the bottom of my heart! You're next :) Shout out to Kendrick (To Pimp a Butterfly), J. Cole (2014 Forest Hills Drive) and the Beethoven Pandora station for the sweet melodies that kept me motivated. To my Chair Barbara Henderson - words can't describe how thankful I am. You encompass the "above and beyond" approach the students in this study spoke about. To my Committee members Jake and Graciela - thank you for your words of encouragement, feedback, and your authenticity. To the students who participated in this study - Thank you for sharing your experience and allowing me to grow as a scholar and professional that is guided by authentic caring and a passion for equity and justice. To all my beautiful people from East Side Salas - I love you. This is for you. Siempre Adelante. 


\section{TABLE OF CONTENTS}

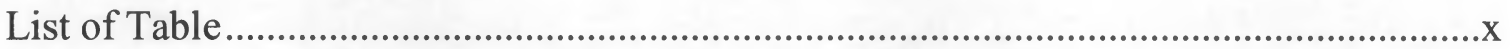

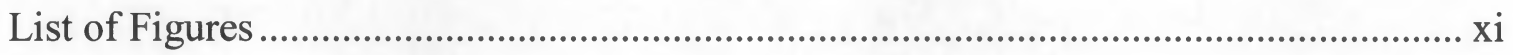

List of Appendices ........................................................................................ xii

Chapter One: Purpose of Study............................................................................

Introduction and Problem Statement .............................................................

Raza Heterogeneity ...............................................................................

Purpose of Study .............................................................................................

Research Questions ....................................................................................13

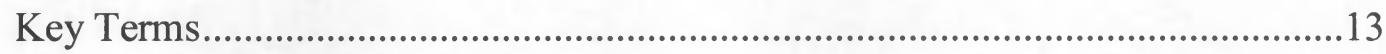

Theoretical Framework .................................................................................. 14

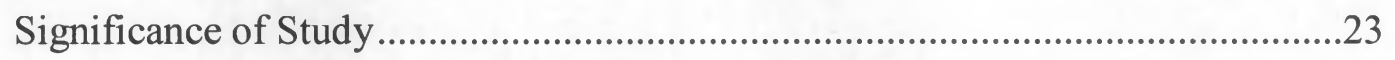

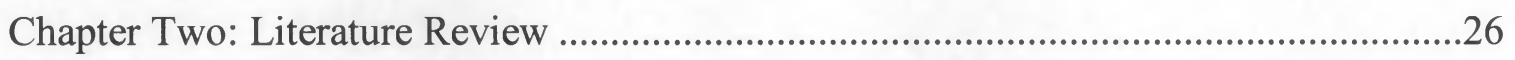

Scope and Structure of Review .....................................................................26

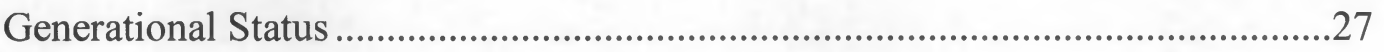

Raza Degree Attainment Contributing Factors.....................................................31

Raza Barriers to Degree Attainment ..................................................................35

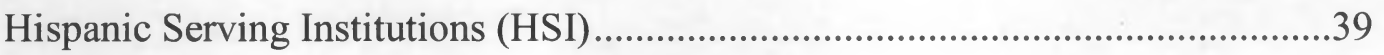

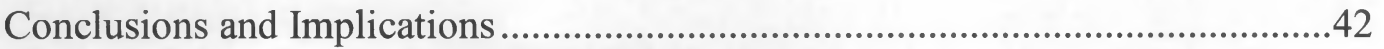




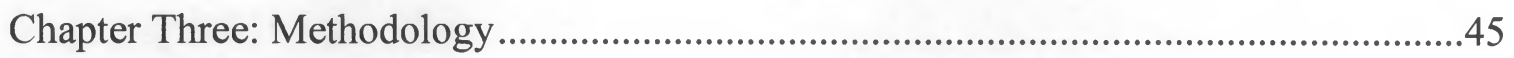

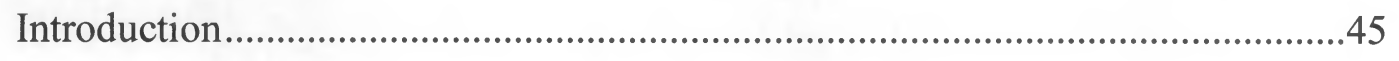

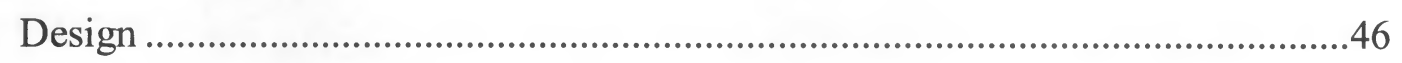

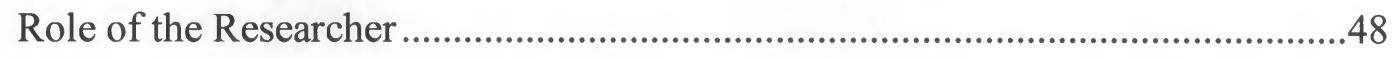

Significance of Research Questions and Sub-Questions ....................................50

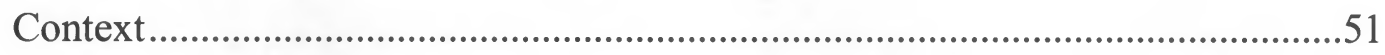

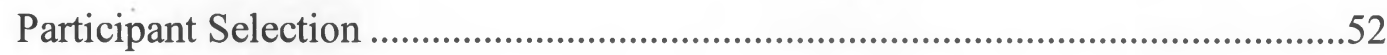

Population and Sample .............................................................................54

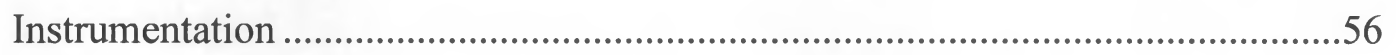

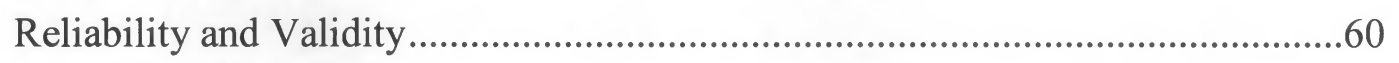

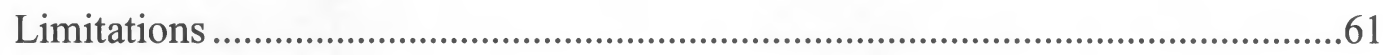

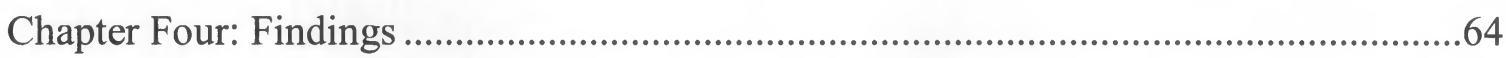

Conceptual Framework ..................................................................................66

Research Question Significance .................................................................69

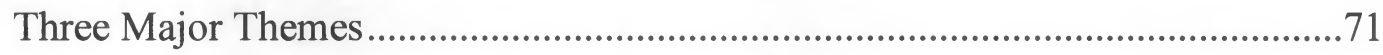

Family Away From Home .......................................................................... 73

Confianza and Mirroring the Home Support System ...............................75

Home Challenges .......................................................................... 78

The Commuter Experience ...................................................................8

Gender and Generational Factors.........................................................83 


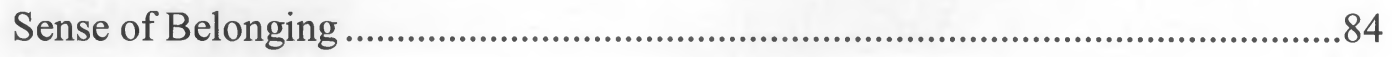

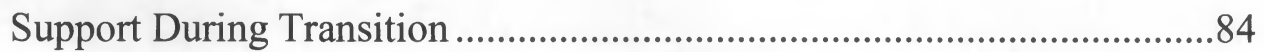

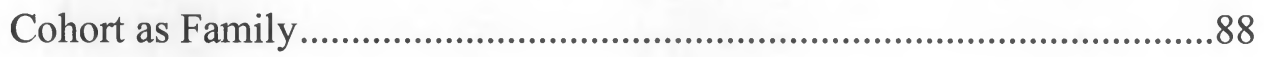

Finding a Connection .................................................................................90

Marketing/Branding of Services ..............................................................96

Internalized Oppression ........................................................................................99

Challenges in Reaching Out..........................................................................99

Fearing the Implications of "Discrete" Services.......................................103

Impact of Pre-College Experiences ............................................................105

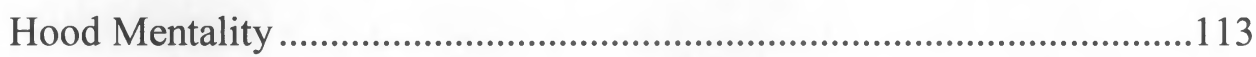

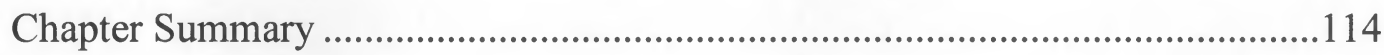

Chapter Five: Discussion and Conclusions.................................................................121

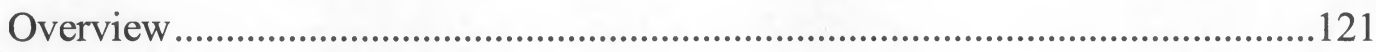

Justification for Generational and Gender Focus..............................................123

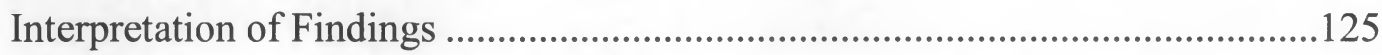

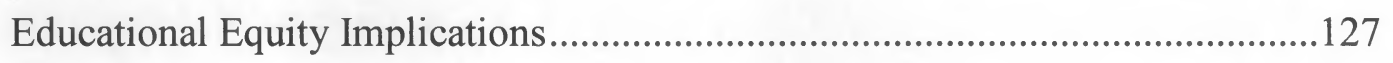

Implications for Theory, Policies, and Research .................................................129

Implications for Institutional Leaders ...................................................................134

Integrate Findings into Campus Diversity Plan ..........................................134

Campus Leadership Welcoming Discussion on Race and Ethnicity ........136 
Implement Latinos Student Success (LSS) Inquiry Model

Culturally Relevant Professional Development

Additional Support for Raza Students Transitioning into the University 141

Establish a Formal Commuter Student Support System

Comprehensive Mentoring Program for Raza Students

Improved Assessment of Raza Student Strengths and Areas of Need.....145

Re-thinking the Way Services are Marketed ..........................................146

Recommendations for Future Research ..........................................................147

Reflections on the Research Process.......................................................... 148

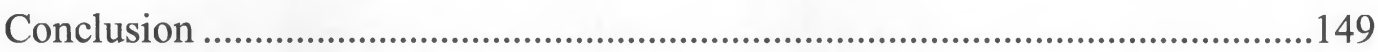

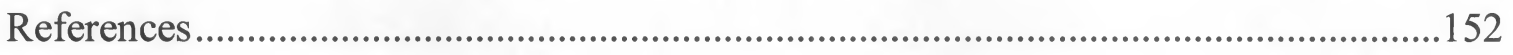

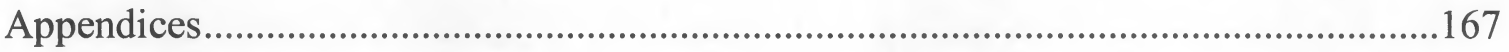




\section{LIST OF TABLES}

Table

Page

1. Profile of Raza Student Population at CCU.................................................53

2. Breakdown of Study Participants..........................................................56

3. Study Themes and Sub-Themes............................................................. 73 


\section{LIST OF FIGURES}

Figures

Page

1. Raza College Student Support Model........................................................66 


\section{LIST OF APPENDICES}

Appendix

Page

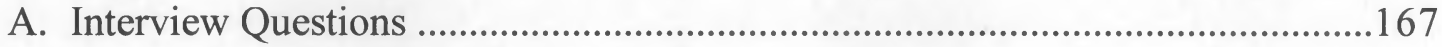

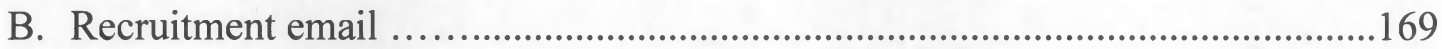

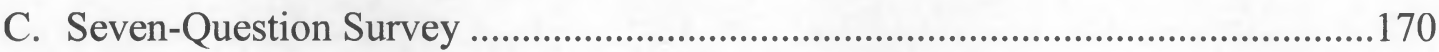

D. Email Sent to Students Who Completed Survey ..........................................171

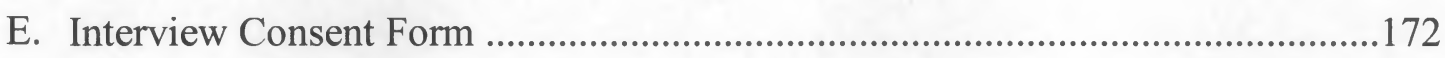

F. Latino Student Success (LSS) Inquiry Model .........................................175 


\section{Chapter One: Purpose of the Study}

Nothing is more central to securing a brighter future for our country than increasing dramatically the number of Latinos we graduate from our colleges and universities over the next decade (HSI Arciniega, 2012)

This study is significant for me on so many levels. As a son of Mexican immigrants who made a number of sacrifices to provide educational opportunities for themselves and their children, I feel it is my duty to somehow contribute to the educational and subsequent economic and social, advancement of marginalized populations in the United States. The role of "educator" has taken on a significant meaning in my immediate family, as both of my parents, one of my younger sisters, and I are "educators" at the pre-school, elementary, vocational, and university levels. As a result, "education" is a hot topic during our family gatherings. We often find ourselves discussing the challenges faced by the students we work with, focusing our conversation on those students who struggle the most within our educational spaces: Raza students. I must note that my use of the word "Raza" is intentional throughout this dissertation due to my belief that traditional words used to categorize Raza, such as Hispanic and Latino, do not encompass the socio-cultural and political identities of indigenous and brown peoples from Latin America. My use of the word "Raza" ultimately aims to serve as a counter-narrative to the deficit-view approach many scholars adopt when examining people of color.

I see myself as someone who has been privileged enough to successfully navigate and educational system that so many times had me doubting myself. When I encountered 
various challenges during my educational career, there were factors and people at play that supported me in ways that I was unaware of at the time, as I wasn't able to realize the significance of their impact until I myself was in a position to serve as a resource and support system for students. Now that I have more than 6 years of professional experience working with first-generation and low-income students in an academic setting, I see the significance that the interactions, words of encouragement, and subtle positive and welcoming messages have on all of our students, particularly those Raza students whom I have a cultural connection with. A majority of the students who fall under the first-generation and low-income category at my home institution are Raza students, a reality that has led me to this research study, in which I hope to contribute to institutional conversations relating to the challenges faced by our students, the cultural capital they bring with them, and the way support systems are contributing to or hindering their educational experience. I believe these conversations can lead to improved graduation rates for all students, particularly those that make up the largest subgroup - Raza students. I am intentionally looking at the intersection of generational status and gender because I feel there is a need to further explore the unique challenges faced by different genders and Raza generational groups, specifically those who identify as first, second, and third generation.

In the last few years, as I dove deeper into this world of education, activism, and intellectual stimulation, I encountered friction as I felt there was a need to find balance between home/family and intellectual/educational identity. I had a desire to stay 
connected to home culture and family, to not forget where I came from, fearing that I would defeat the purpose of my educational and professional journey. The development of this new intellectual/educational identity has challenged the developed home identity I am most familiar with. The resulting "balance of identities" and the experience encountered throughout this process has been quite a ride, and thus I find the need and the desire to explore how Raza students at my institution are dealing with this phenomenon. My interest is not only based on my desire to improve educational outcomes for Raza and all students at my institution; it is also based on my own continued struggle to identify and take advantage of the support systems that will help me continue on a positive path in my professional, educational, and spiritual journey. While Raza students will most surely encounter additional challenges as they persist in their educational careers, it is my hope that this work will have a positive impact on them, if anything by helping them normalize the challenges that Raza students face as they pursue a college degree and then move on to becoming civically and democratically engaged citizens of the $21^{\text {st }}$ century.

\section{Introduction and Problem Statement}

It has been over six years since President Obama first demonstrated his commitment to an educated $21^{\text {st }}$ century workforce by pledging that the United States would have the highest proportion of college graduates in the world by 2020 (Obama, 2009). According to Douglass (2010), meeting this pledge would require a huge increase 
in the number of degrees that are awarded - at least an additional 8 million baccalaureate degrees over an 11 year period. A similar degree attainment objective has been pledged by the Association of Public and Land-Grant Universities (APLU) and the American Association of State Colleges and Universities (AASCU) through "Project Degree Completion," an initiative with the commitment of 500 four year colleges and universities to increase degree completion rates by 3.8 million, ultimately leading to $60 \%$ of U.S. adults having a university degree by 2025 (Lee Jr. \& Keys, 2013). Additional college completion initiatives have been developed through the College Board and other well respected educational groups such as Excelencia in Education, highlighting the significance of degree attainment on our country's ability to increase its position in the $21^{\text {st }}$ century global economy.

For the nation to meet this goal, groups who are currently underrepresented in higher education must increase their rate of college degree attainment. Considering the Raza population will make up $31 \%$ of the U.S. population by 2060 (Santiago, Galdeano, \& Taylor, 2015), the degree attainment of Raza students will have a significant impact on whether President Obama's 2020 pledge is made a reality. De Leon (2005) found that Hispanic enrollment in our nation's colleges/universities will continue to increase faster than any other ethnic group - $39 \%$ by 2017 , emphasizing the need to ensure that students who are enrolled in higher education encounter a system of supports and encouragement that can lead to graduation. Lee Jr. and Keys (2013) reaffirmed that "Project Degree Completion" cannot reach its ambitious goal without a significant increase to the college 
access and degree attainment rates of Raza students.

Although the overall number of Raza graduating with degrees has steadily grown, the numbers are still a concern when considering the significant growth of the Raza population in the United States. Currently, out of 100 Raza students who enroll in Elementary School, only 10 will graduate with either a 2- or 4-year higher education degree (Dorame, 2012). This reality has led to a degree attainment rate of $13 \%$ for the U.S. Raza population above the age of 25 (U.S. Department of Education, 2011). The significance of this problem is further highlighted by data showing there is a $14 \%$ college completion gap between recent first year cohorts of full-time Raza students versus their non-Raza white counterparts (Santiago \& Soliz, 2012). Therefore this dissertation, which explored how better to support the degree attainment of Raza students, provides a significant contribution to the literature, and to the practical reform measures that higher education must take to support Raza students.

Of specific concern is the Mexican-American (MA) college completion rate, which is the lowest among Raza subgroups (Cerezo, Lyda, Beristianos, Enriquez, \& Connor, 2012). In 2013, only $10 \%$ of MAs above the age of 25 had earned a baccalaureate degree (Gonzalez-Barrera \& Lopez, 2013). While this $10 \%$ is not significantly lower than the $13 \%$ Raza degree attainment that was described above, Raza of Mexican descent made up close to two-thirds of the U.S. Raza in 2012 (GonzalezBarrera \& Lopez, 2013), highlighting the significance of this group in discussions about reaching Obama's 2020 college completion goal. Further, there is the issue of gender 
specific degree attainment challenges within the Raza population; Raza males have the lowest percentage of educational attainment of all subgroups (Lee \& Ransom, 2011). In the United States, $8 \%$ of Raza males above the age of 25 have a four year degree as compared to $20 \%$ of white males (U.S. Census Bureau, American Community Survey, 2011). Additional data from (Goya, Vance, \& Serros, 2014) found less than $20 \%$ of Raza males who go to college end up graduating with a degree, a troubling statistic that sheds light on the challenges Raza males are specifically facing in the college environment. The low degree attainment rates of Raza of Mexican descent are especially significant in our state of California, with $83 \%$ of Raza in the state being of Mexican descent (Valliani, 2015). Overall, as the college enrollment rates of Raza students continues to increase increased from 54\% - 70\% from 2002 to 2012 and is projected to increase $27 \%$ from 2011-2022 (Santiago et al., 2015) there is a need to examine ways to ensure the increase in enrollment leads to an increase in college graduates.

The U.S. Department of Education and other government agencies have recognized the need to invest in the educational attainment of the increasing Raza population. At the college level specifically, the "Hispanic Serving" label was created during the 1992 Higher Education Act (HEA) Reauthorization (Malcom-Piqueux \& Bensimon, 2015) in order to allocate additional funding for institutions to strengthen infrastructure and provide additional support to the Raza student population. While the Hispanic Serving Institution (HSI) label was introduced in 1992, it wasn't until 1998 that Title $\mathrm{V}$ funding was made available to provide assistance to institutions with a Raza 
student population above $25 \%$ (Hurtado \& Ruiz, 2012). Since then, the number of HSIs has continued to grow as more and more Raza students enroll in college - from 20032013, the number of HSIs increased from 238 to 370 nationwide (Santiago et al., 2015). Currently, HSIs provide a great opportunity for addressing issues of Raza degree attainment as over $59 \%$ of Raza college students were enrolled in designated HSIs in the 2012-2013 academic year (Santiago et al., 2015). In providing funding for HSIs, the government seems to acknowledge the economic and social implications of increasing college degree completion rates for Raza. Members of the Hispanic Association of Colleges and Universities (HACU) have emphasized this need for additional funding for our country to address our $21^{\text {st }}$ workforce needs (Galdeano, Flores, \& Moder, 2012). According to Malcom-Piqueux \& Bensimon (2015), “...these institutions are critical to increasing educational opportunity and attainment among the [Raza] diaspora" (p. 2). Just as there has been a focus on ensuring students graduate from high school, there now needs to be a stronger focus on college student persistence and degree attainment (De Leon, 2005). For HSIs, "it is not enough to be a Hispanic-Serving Institution, it is imperative to be a Hispanic-graduating institution" (Arciniega, 2012, p. 156).

Central Coast University (CCU) gained its HSI status in 2008 and currently has a Raza student population above $36 \%$, a number that will continue to rise and will ultimately lead to Raza students being the ethnic majority at the institution. CCU aims to "be distinctive in serving the diverse people of California, especially the working class and historically undereducated and low-income populations," with a specific focus on 
serving underrepresented students from the surrounding Tri-County area. The economy of this area relies heavily on Raza agricultural laborers to sustain a multi-billion dollar agricultural industry. The university's growth plan projects enrollment to reach about 12,000 by the 2021-22 academic year. Given existing levels of enrollment that are already placing stress on the institution, CCU has declared "impaction," a move that will prioritize enrollment for Vision and Tri-County students. Thus, CCU will likely see an increase in the number of Raza students enrolled at the institution, further highlighting the need to ensure that support systems are in place to assist these students in their pursuit of a college degree. In addition, it is necessary to consider the significance of the financial limitations the California State Universities (CSUs) have experienced over the past 10 years. As a result of state general fund allocations dropping more than $40 \%$, the CSU tuition has increased over 150\% since the 2003-2004 academic year (Valliani, 2015). The increase in tuition costs has led to more financial strain and less support for students at the CSU. Raza students specifically, with over $55 \%$ of students entering their freshman year of college as remedial in writing and math (Valliani, 2015), have been negatively impacted by the additional financial stress caused by higher tuition as well as less support services to help them address academic and other developmental needs. In an effort to increase graduation rates, CCU has adopted Strategic Themes designed to enhance Student Success (Theme 1) and Academic Excellence (Theme 2). The Student Success theme calls for strategies that improve retention and graduation rates, improve advising, support development of the whole student, and improve 
internship, career and graduate school placement rates. To address the Raza college completion specifically, prominent CCU administrators, faculty, and staff have come together over the past year to create an HSI Co-Op that is focused on providing the institution with recommendations to effectively address the educational and developmental needs of Raza students as well as implementing HSI best practices that will impact all students. Further, the institution conducted a campus climate survey in the Spring 2015 semester as part of efforts to create a more inclusive institutional environment that is focused on supporting the academic and personal development of students. The survey focused on measuring the following areas: discrimination and harassment; positive cross-racial interaction; negative cross-racial interaction; institutional commitment to diversity; academic validation in the classroom; satisfaction with diverse perspectives; student financial difficulty; interpersonal validation; and sense of belonging. The result of the surveys were summarized in the Fall 2015 semester and will lay the foundation for the development of a CCU campus diversity plan that will influence the institutions approach to diversity and inclusion for the foreseeable future. These initiatives are aligned with recommendations from Hurtado and Ruiz (2012), emphasizing that addressing the needs of students at an HSI institution requires alterations in campus systems that can impact the institutional culture and policies.

\section{Raza Heterogeneity}

Due to the heterogeneity of the Raza population (Ayala, 2012), as well as the ambiguous findings that may result in examining student populations at a number of 
institutions (Zepke \& Leach, 2005), this study will explore the support systems that hold value for a multi-generational Raza population at a single institution, one that is designated as an HSI institution due to its Raza population being above 25\% (Mendoza, 2012). In an effort to examine the heterogeneity of the Raza population, it is important to focus on generational status and the impact this component may have on utilization of support systems. Unclear findings on the role of generational status on academic performance further justify the need for additional examination (Aguayo, Herman, Ojeda, \& Flores, 2011; Torres, 2003). Past research has emphasized the need to examine institutional factors, such as the support systems available to students, who provides them and how, how those factors play out for students, how they perceive support systems, what has worked and what has not, and what additional support they would like to see (Reason, 2009). This study will add to the research by focusing on how those support systems impact a multi-generational Raza population at an HSI.

The examination of HSI institutions appears to be a top priority for the California State University (CSU) system. The CSU currently has 15 institutions designated as HSI's and currently, $33.4 \%$ of CSU students are Raza, the largest of all ethnic groups. In addition, two CSU presidents were selected in 2014 to serve on the governing board of the Hispanic Association of Colleges and Universities (HACU), to assist HACU in improving the post-secondary success of Raza students (Thara, 2014). 


\section{Purpose of Study}

The proposed research focuses on exploring the unique student support experiences of the multi-generation Raza population at the 4-year HSI institution. The goal will be to identify the support systems that students cite as beneficial in their pursuit of a university at that specific HSI institution. While researchers have examined various educational problems for a large group of Raza students, there is limited research that examines problems based on specific population subgroups, in this case a multigenerational Raza student population at a single institution. There have been calls for further examination of Raza subgroups in order to learn how their culture-specific contexts impact their development (Gloria et al., 2009). There have also been calls for research exploring the impact of individual student attributes and socio-demographic variables on the academic experience on specific demographic groups (Reason, 2009), and how these cultural and socio-demographic variables combine with institutional components as students come in contact with systems of support. The need for additional studies that examine the various services and environments that students encounter on campus has been recommended by Reason (2009). Aguayo et al. (2011) expanded on this recommendation by encouraging studies that examine the role of biculturalism and generational status on student achievement in postsecondary education.

In regards to generational status, literature is not conclusive about the impact of generational status on academic performance in postsecondary education. For Raza specifically, studies have found that $3^{\text {rd }}$ generation Raza perform better than $1^{\text {st }}$ 
generation students (Aguayo et al., 2011) while others found that $1^{\text {st }}$ generation students are more motivated and perform at a higher level (Kim \& Chao, 2009). Other studies have found that $1^{\text {st }}$ generation students with immigrant parents encounter more challenges than students with parents born in the United States, and that generational status has more of an impact on academic performance than does socioeconomic status (Alon, Domina, \& Tienda, 2010). There is also the issue of a drop in ethnic loyalty from $1^{\text {st }}$ to $2^{\text {nd }}$ generation (Torres, 2003) that deserves specific attention, particularly as it relates to the role that ethnic identity plays on academic outcomes (Guyll, Madon, Prieto, \& Scherr, 2010). Aside from generational status, researchers have emphasized the need to examine the heterogeneity of the Raza population when implementing studies on academic persistence due to the difference in cultural practices within the multi-generational Raza population. Gloria et al. (2009) recommended that research focus on specific Raza subgroups in order to gain more reliable findings. Further, there is need for research that focuses on exploring issues within a single institution due to the ambiguous findings that could result from multiple institution studies that may not account for differences in institutional factors and population (Zepke \& Leach, 2005). As Kuh and Love (2000) discuss, all institutions have unique customs and traits that impact students in distinctive ways. This research ultimately aims to provide perspective to the discussion on the role of support systems in addressing college degree attainment issues for various student populations. 


\section{Research Questions}

The research questions for this study are: 1) How does family influence and the life and educational experiences of a multi-generational Raza student population across

genders impact students' perception of support? 2) How does a multi-generational Raza student population across genders perceive the effectiveness of existing institutional supports at an HSI? 3) What additional supports do multi-generational Raza students across genders identify as necessary to succeed at an HSI?

\section{Key Terms}

For the purposes of this study, the following key terms require definition:

Hispanic: According to O'Meara (2012), the term Hispanic is used to identify populations with roots to a Spanish speaking country: central/south America, Mexico, Caribbean Islands, or Europe, USA, and Puerto Rico. For the purpose of this research study, the term "Hispanic" will encompass multi-generational populations with connections to Mexico and the Central American countries of Guatemala, El Salvador, and Honduras.

Hispanic Serving Institution (HSI): Federal Program designed to assist colleges or universities in the US that attempt to assist first generation and low-income Hispanic students. Institutions must have a Hispanic student population above $25 \%$ in order to be designated as an HSI (Mendoza, 2012). 
Generational Status: Determined by factors that include where students are born, where parents are born, and where grandparents are born (Becerra, 2010).

- First generation or foreign born: Born outside of US to parents who were not citizens at the time (Taylor et al., 2013).

- Second generation: Born in the US with at least one first generation (immigrant) parent (Taylor et al., 2013).

- Third Generation: Born in the US, with both parents born in US (Taylor et al., 2013).

Raza: Defines a broader socio-cultural and political identity, which includes the standpoint of Indigenous and colonized Mestizo/Brown peoples from Latin America (Zavala, 2013)

\section{Theoretical Framework}

The Cultural Wealth Model (Yosso, 2005) provides a foundation for understanding the challenges multi-generational Raza students encounter in higher education while also providing a framework for addressing Raza students' educational and developmental needs through addressing their capital in six areas:

- Aspirational: Encouraging students to have high educational aspirations

- Linguistic: Building on the language and communication skills Raza students bring with them to the institution

- Familial: Helping students leverage the experiences and values rooted in their precollege environment 
- Social Capital: Helping students stay connected to their communities and home culture as a way to positively impact their academic persistence

- Navigational: Providing students with the capital that will assist them in successfully navigating the various institutions they encounter

- Resistance: Providing students with knowledge and opportunities for developing as civically engaged citizens According to Yosso (2005), the Cultural Wealth model provides a counternarrative to the deficit view that is often associated with students of color, specifically those that come from communities that are identified as disadvantaged. The model calls for critical educators to tap into the cultural wealth of students as part of a move towards recognizing the experiences and knowledge of students of color as a resource that will assist them in navigating the educational institution, while also developing in them a mindset based on social justice and equity.

This Cultural Wealth Model is significant for this study as it provides a frame for addressing the challenges Raza students encounter as they navigate educational institutions that take a deficit view of the cultural and social capital students bring with them. As student perceptions of support are examined, the Cultural Wealth Model will serve as a lens to identify potential solutions to address the support needs of multigenerational Raza students. Yosso's (2005) Cultural Wealth model includes in its foundation elements of Latina/o critical race theory (LatCrit), which ensures the 
examination of race, culture, class, behavior, and language as part of the cultural wealth exploration process (Yosso, Smith, Ceja, \& Solorzano, 2009).

This study will also aim to build on the Bi-cultural Orientation Model (BOM) that was developed in a study that explored the ethnic identity development of ten Latina/o college students in their first two years (Torres, 2003). According to the BOM, there are four orientations:

- Bicultural orientation: indicates a comfort level with both cultures

- Latino/Hispanic Orientation: indicates great comfort with the culture of origin

- Anglo Orientation: indicates a greater comfort with the majority culture

- Marginal orientation: indicates discomfort with both cultures and may indicate conflict within the individual As part of his study, Torres (2003) included "situation identity and influences on change," to account for the impact of a student's home pre-college environment, the role of family, generational status, and student self-perception of their status in society. The $\mathrm{BOM}$ is significant due to the belief that disconnect to the dominant culture, in this case an institutional culture, impacts a students' level of comfort, which can then ultimately impact the students' decision to seek various systems of support. This study will utilize the BOM to provide further insight into how the various cultural orientations play out in the lives of multi-generational Raza students and whether those orientations have an impact on a student's willingness to seek support services.

Just as Raza from past and present generations have navigated from one place to 
another in the physical sense (immigration), many students are now navigating "from here to there" in the realms of identity and culture as they balance their home identities with their college identities. Raza students often move through various structures that require them to receive validating words or recognition in order to improve their sense of belonging and comfort within structures like the 4-year institution.

According to Saldana (2012), research on "identity" can be problematic as it can mean so many things and be defined in so many ways. All that can be definitively said about identity is that it "exists by how it is defined" (p.62). It is important then to clarify how identity is being defined within the context of this study. In this case, the research considers how the "sense of self" (Saldaña, 2012, p. 62) of Raza students from different generational statuses plays a role (if any) in how their perceive support systems. Identity is addressed through questioning about support systems and experiences that have led students to utilize or not utilize support services. The way students "orient" themselves in conversation reveals key information about institutional, personal, intellectual components that must be examined, and it is important to focus on the ways a student's identity are disclosed in their telling of autobiographical stories (Wood \& Kroger, 2000).

\section{Hegemony}

The concept of hegemony is significant for this study as it addresses the dominant mindset that is reinforced in the $\mathrm{K}-16$ educational system many Raza students encounter. Apple (1990) presents the concept of hegemony to show how the dominant class in the 
United States is able to manipulate and control their submissive counterpart, the oppressed. One might ask: why is it necessary for the dominant class to employ hegemony? Hegemony is necessary for the dominant class to maintain cultural control and grow the economic establishment that they benefit from. This hegemony is made up of certain beliefs (theory of cultural deficiency), and practices (banking model of education, subtractive schooling) that are based on the ideology of dominance, leading to a number of limitations for the oppressed. A significant limitation is the inability to improve their educational experience and subsequent social and economic conditions, a reality that has psychological implications: "hegemony acts to 'saturate' our very consciousness, so that the educational, economic and social world we see and interact with, and the commonsense interpretations we put on it, become the only world" (Apple, 1990, p. 5). The psychological condition leads to the development of a consent that normalizes and justifies the oppressive conditions in place. The hegemonic relationship is then sustained through a process Apple (1990) calls the "Selective Tradition": the structure of dominance is passed on as the norm from generation to generation. Lugg (2003) argues that this process is ingrained into our very being as a country and that much of the equity issues encountered in schools are manifested by a constitution and governmental institution that inherently discriminates based on race, gender, sexual orientation, and class. It is thus important to examine the systems that contribute to this hegemony and inequality among Raza students. 
The challenges Raza students encounter in educational institutions are often justified by a school curriculum that is set up to discredit their linguistic, cultural, and intellectual capital because it is not directly aligned with the "one size fits all" curriculum that mainstream educational institutions utilize. Ultimately, this subtractive schooling process robs students of the social and cultural resources that the need to stay grounded (Valenzuela, 1999). Further, as Ayala (2012) emphasizes, it is necessary to consider the significance of racial layers that impact the educational attainment of Raza students. Ultimately, the educational issues experienced by Raza students are works of a culture of power that is founded on a set of values and judgments that has resulted in a class-based society where certain groups of people, primarily marginalized people of color, do not have access to the tools, language, and resources of the culture of power (Calabrese Barton \& Yang, 2000).

\section{Invisibility}

For many Raza students, "balancing identity" is particularly problematic. Many of them deal with a sense of invisibility as they learn to navigate in academic and professional contexts, but this is central to the question of identity development (Reddick \& Saenz, 2012). Their experience navigating the various structures of an institution is often not validated by campus officials and in some cases, the students home support system, who unintentionally reinforce barriers that lead to additional academic and adjustment challenges for the student (Rodriguez, 2012), an issue that may impact a Raza student's decision to seek and/or utilize the specific institutional support systems needed 
to be academically successful. The concept of "invisibility" addresses the feelings of exclusion and lack of acceptance that many Raza students face as they navigate educational and professional environments.

\section{Recognition \& Validation}

On the opposite end of this concept of invisibility is a concept of recognition, which can potentially address the impact of the "invisibility" discussed above. Adopting a "lens of recognition" (Rodriguez, 2012) highlights the need to acknowledge, recognize, and legitimize the presence of Raza college students. The work of "contextualizing their experiences in a larger struggle for voice, identity, and existence" (p. 4) involves engaging "in an exercise" of "acknowledging the social, political, and economic conditions that plague marginalized communities with substandard schools" (p. 5). For Raza students specifically, Rodriguez highlights the power of a pedagogy of recognition that includes relational (how youth are greeted), curricular (how their knowledge and experiences are affirmed), contextualizing (understanding out of school experiences), pedagogical (instruction as linked to student realities), and transformative (critical stance toward educational goals) recognition.

A key component of the pedagogy of recognition includes the need to validate the student experience in the various domains they encounter. The theory of validation (Rendón, 1994) highlights the need to instill in students the belief that the experiences and knowledge they bring to the institution is significant and valuable. Rendón and Muñoz (2011) argue that when students experience validation consistently throughout 
college, such experiences are key in assisting students to become involved, seeking support, and realizing they are capable of learning and achieving their goals. When students do not experience validation, they are confronted with invalidation, a phenomenon which Rendón (2009) argues is a form of oppression, in that it is "a way that people in power exert dominance over others" (p. 94). In the educational setting, invalidation takes place when students encounter instances of discrimination that can also take the form of micro-aggressions.

\section{Micro-aggressions}

The lack of recognition felt by many Raza college students is often impacted by various micro-aggressions that lead to feelings of withdrawal, self-doubt, and poor performance (Yosso et al., 2009). For those Raza students with a strong ethnic identity, micro-aggressions and stereotypes can place extensive pressure and stress on them as they struggle to find a balance in their professional work. Typically, students with a stronger ethnic identity encounter higher instances of discrimination due to their own awareness of the stereotypes associated with their ethnic and cultural group (Castillo et al., 2006). The stereotypes, which include messages that suggest Raza are less likely to achieve academically (Yosso et al., 2009), along with the pressure caused by media portrayals of Raza, have led to excessive pressure and stress for Raza college students as a result of feelings of self-doubt that lead to Raza students questioning whether they belong in the institution. This ultimately leads to a sense of alienation within the institutional climate, as students are less likely to reach out to available support systems 
(Schwartz, Donovan, Guido-DiBrito, 2009). Since there have been calls for HSIs to lead the way in addressing the structural and oppressive conditions that are found in the U.S. system of higher education (Greene \& Oesterreich, 2015), the need for additional HSI research that is critical of these conditions is necessary, with the goal of having HSIs lead the way in disassembling the institutional practices and patterns that sustain discriminatory practices in educational institutions.

An additional way to understand the problems associated with Raza utilization of support system is through theories on student involvement and persistence. According to Kuh and Love's (2000) culturally receptive theory of college student persistence, the precollege experience of students of color serves as the foundation for their college experience. The researchers hypothesized that a student's connection to his community and his ability to negotiate the differences between his home culture and the culture of their academic institution shape the student's ability to remain in higher education. Based on this theory, Raza students are most likely to acculturate to a university if they are satisfied with their academic major and participate in student groups that are culturally relevant and support their goals of college degree attainment.

While the Torres (2003) study provides a framework to examine the issue of Raza students and support services, the study conclusion cannot be applied to a vastly different Raza population. Torres' study took place on the East Coast with 12 students, a relatively small study sample, from Mexican, Puerto Rican, Cuban, Venezuelan, Colombian, and Central American backgrounds. Further, I believe there are different factors that 
influence the Raza college experience on the East Coast versus the West Coast. Torres's study took place utilized a relatively small study sample (12 students) at an institution that was not an HSI, with only a $5 \%$ Raza student population. The heterogeneity of the Raza population (Ayala, 2012), as well as the ambiguous findings that may result in examining student groups at a number of institutions (Zepke \& Leach, 2005) are two factors that have been discussed in research on Raza student performance. Therefore, this study is unique in that it will examine the support systems that multi-generational Raza students perceive as helpful in their pursuit of a degree at a designated HSI public institution on the West Coast.

\section{Significance of Study}

HSI institutions are in a position to play a role in a new institutional culture that is addressing the unique needs of Raza students. Some have argued that HSIs have not done a good job of living up to the "Hispanic Serving" label and instead engage in practices that can be seen as continuing to reinforce structural inequalities and promoting a classist and racist higher education system (Greene \& Oesterreich, 2015). HSIs continue to receive millions of dollars in funding without an accountability structure focused on improving educational outcomes and degree attainment rates for Raza students. In addition, only a small percentage of HSIs have embraced an institutional character focused on changing systems and policies that would lead to improved academic success for Raza students. Similarly, Malcom, Bensimon, \& Davila (2010) argue that HSIs do 
not live up to their potential to impact the social justice landscape and "providing equitable access to the public good" (p.2). Appropriately so, these scholars view the HSI label as an opportunity to change the social and economic conditions for Raza in this country as well as the social and economic landscape as a whole. As Lee Jr. \& Keys (2013) pointed out, "It is critical that HSI institutions assess how well they are serving their Latino student populations (p.6). There is a need for additional research on specific actions and evidence that highlights how HSIs are improving Raza student outcomes and how institutional culture has developed to support the achievement of Raza students (Hurtado \& Ruiz, 2012).

Another problem highlighted in research is that on average, 4-year HSIs have lower 6-year graduation rates than their Predominantly White Institution (PWI) counterparts $-31 \%$ compared to $39 \%$ for PWIs (Greene \& Oesterreich, 2015). While some might argue that HSIs graduate all students at lower rates than PWIs in general, if HSIs are to live up to their label of improving educational outcomes for Raza students, there is a need for additional examination of HSI institutions and the various components that impact student persistence and graduation rates. Ultimately, it is my goal to contribute to the emerging field of HSI research in an effort to highlight the experiences and needs of multi-generational Raza student groups.

On a larger scale, the proposed study is also significant due to the equity implications tied to the degree attainment rates of Raza college students. There are enormous financial benefits from an increase in the number of Raza who graduate from 
college: $\$ 7$ billion more dollars in federal tax revenue and $\$ 480$ billion more in social security revenue for every ten year group of students (Becerra, 2010). As the Raza population continues to increase, their role in the democratic process and the labor force will be critical to our nation's prosperity. Raza will account for 60 percent of the country's population growth from 2005-2050 (U.S. Department of Education, 2011).

At the institutional level, the study's focus on gaining a better understanding of the support systems that can have a positive impact on the educational outcomes and experience of a multi-generational Raza student population is aligned with campus efforts to address issues of inclusivity, as evidenced by the institutions focus on conducting a campus climate study that will lead to the development of a campus diversity plan. Similarly, as a practitioner-scholar, I believe the study findings can have a positive impact on my own work with all underrepresented and low-income students at my home institution. As I continue to move in the professional direction of administration, I believe the study findings will provide me with data to support calls for institutional transformation and the adoption of policies and systems that promote academic success and personal development for all students, not just those of a Raza background. 


\section{Chapter Two: Literature Review}

\section{Scope and Structure of Review}

This dissertation examines the role of pre-college life and educational experiences on Raza students' perceptions of support, as well as the supports that multi-generational Raza students across genders utilize and/or perceive as effective at an HSI 4-year institution. This topic has received scant attention in the literature. For that reason, this review of relevant literature will synthesize recent literature that examines the various sub-topics that make up the focus of this study. The review will first provide a summary of the scholarly database search process as well as keywords that were the focus of the search. I will then summarize research on Raza college performance based on generational status. Factors that contribute to and/or hinder college completion for Raza students will then be examined to identify supports and barriers that have been previously highlighted. In addition, past research on HSIs, specifically studies that focused on aspects of Raza degree completion at HSIs, were examined in an effort to explore best practices and challenges faced by HSIs. Finally, the review of literature concludes with a discussion of limitations of the existing literature in order to validate the proposed methodological approach for researching this particular problem.

KEYWORDS: first generation, persistence, retention, support, Hispanic Serving Institution (HSI), Latino, Hispanic, Mexican-American, Raza, Chicano, graduation, and degree attainment. 
In order to execute the review, I accessed a number of database search engines that including Academic Search Complete, LexisNexis Academic, and Google Scholar. Sources were selected based on their relevance to the topic being studied, date of publication (articles from the past five years), methodology employed, connection to the research questions of this study, and the keywords included on the first page of the article. Relevance was often determined by reading the article abstract and conclusion as well as skimming the findings section. All of the keywords included above were searched in various combinations in an effort to access as many appropriate articles as possible. The various searches took place over three years with the focus and keywords changing as the study focus developed into the current project. Some studies were utilized in for literature review purposes only while others were selected due to methodological relevance, implications based on findings, and/or the keyword focus. In addition, I reviewed a number of policy briefs, working papers, book compilations, and reports that included data on the Raza population, their educational attainment, income status, generational status, etc.

\section{Generational Status}

A review of relevant literature identifies discrepancies in the academic performance of Raza across generations. Data from the Pew Hispanic Center (GonzalezBarrera \& Lopez, 2013) reported that Mexicans born in the United States earn a college degree at three times the rate of Mexican descent students who are immigrants, $15 \%$ to 
$6 \%$ respectively. In contrast, Portes and Rumbaut (2001) found that $3^{\text {rd }}$ generation Raza students have lower levels of optimism and expectation than their $1^{\text {st }}$ and $2^{\text {nd }}$ generation counterparts. Alternative research finding may support reasons why those first generation students specifically have higher levels of optimism - Owens and Lynch (2012) found that $1^{\text {st }}$ generation immigrants do not seem to be adversely affected by stereotypes that depict them as inferior. Yet they find that stereotyping begins to have an impact on $2^{\text {nd }}$ generation students, possibly because these students developed a level of awareness to stereotypes, as they have had more experiences in identity impacting domains than $1^{\text {st }}$ generation students. The lack of awareness to stereotypes for $1^{\text {st }}$ generation students could be impacted by the fact that many $1^{\text {st }}$ generation students live in environments where a similar ethnic immigrant population is present, minimizing the types of experiences and interactions that have a negative impact on their development. Owens and Lynch (2012) were quick to point out that other factors such as income and growing up in environments with fewer people of one's own ethnic background who have been academically successful have an impact on how well students are able to stay optimistic throughout their educational journey.

The findings presented by Owens and Lynch (2012) are supported by research that highlights challenges faced by $3^{\text {rd }}$ generation students. According to Becerra (2010), $3^{\text {rd }}$ generation students were more likely to be susceptible to developing a greater perception of barriers to college completion. While many $3^{\text {rd }}$ generation students are more acculturated, there appeared to be a connection between acculturation and perceived 
awareness of barriers, possibly a result of the additional time spent in an environment where they encountered discrimination. Although $3^{\text {rd }}$ generation students are encountering unique challenges, Aguayo's et al. (2011) study at an HSI institution found that $3^{\text {rd }}$ generation students performed at a higher level than $1^{\text {st }}$ generation students. Since this study took place in a town with an $86 \%$ Raza population and bordered Mexico, these $3^{\text {rd }}$ generation students may have faced fewer instances of discrimination and stereotypes. The study also highlights the fact that the level of optimism of $1^{\text {st }}$ generation students did not necessarily transferred to an increase in college completion. Ayala (2012) found that higher optimism did not necessarily lead to degree attainment. While optimism might be high for $1^{\text {st }}$ generation students, they are often faced with additional challenges that deter degree completion, such as familial and financial commitments (Dorame, 2012). In order to help bridge the connection between optimism and academic success among $1^{\text {st }}$ generation students, many researchers (Cerezo et al., 2012; Aguayo et al., 2011; Yosso et al., 2009; Solorzano, Villalpondo, \& Oseguera, 2005) recommend that institutions establish spaces where these students can freely express themselves, as will be discussed further below. Overall, research has highlighted the unique challenges faced across generations, reinforcing the need to examine the supports that would be most effective for each generational group as they continue along their educational journey.

Another issue that must be discussed is the significance of students who label themselves as "Hispanic" versus those that use labels such as "Chicano" or "MexicanAmerican". In many cases, the preferred identifying term for this student group is 
reflective of life experiences that have impacted their identity in the various Raza and American cultural realms they navigate (Torres, 2003). The term "Hispanic" is not used exclusively to identify the Raza population that is the focus of this study as it carries a historical negative connotation for the Raza population that is of Mexican descent. Gutierrez (2009) places the use of the word "Hispanic" within a historical context -- the term came to exist as a way for people to self-identify with origin to Spain while negating Mexican or Indigenous origins. As a result, using the term "Hispanic" may be offensive to people of Mexican descent who do not identify with the term. It is important to understand that people of Mexican origin represent close to two-thirds of the Raza population in the United States (Gonzalez-Barrera \& Lopez, 2013). According to Rumbaut (2009), the classification of "Hispanic" or "Latino" is a phenomenon that is specific to the United States, as no Raza subgroup was identified as "Hispanic" or "Latino in their countries of origin. As mentioned in Chapter one, my use of the word "Raza" is intentional throughout this dissertation due to my belief that traditional words used to categorize Raza, such as Hispanic and Latino, do not encompass the sociocultural and political identities of indigenous and brown peoples from Latin America (Zavala, 2013). My use of the word "Raza" ultimately aims to serve as a counternarrative to the deficit-view approach many scholars adopt when examining people of color. 


\section{Raza Degree Attainment Contributing Factors}

One way to understand the problems associated with Raza degree attainment is through theories on student involvement and persistence. According to Kuh and Love (2000), the precollege experience of students of color serves as the foundation for their college experience. The researchers hypothesized that students' connection to their community and their ability to negotiate the differences between home culture and the culture of their academic institution shape students' ability to remain in higher education. Based on this theory, students from various Raza subgroups are most likely to acculturate to a university if they are satisfied with their academic major and participate in student groups that are culturally relevant and support their goals of college degree attainment. Within the context of an HSI institution, a student's belief of self has been associated with an increase in acculturation as well as academic performance (Aguayo et al., 2011).

A Raza students' ability to ask for help and guidance may be partly dependent on their level of acculturation, that is, their ability to integrate into the host campus community (Aguayo et al., 2011). That research found that the level of acculturation of Raza students has an impact on their academic success and ambition, often leading to improved educational and professional results. Specifically, students who were connected to both their home as well as institutional culture had better self-efficacy and an increased confidence to do well in school. As students feel more comfortable navigating within their home and academic cultures, their perceptions of social and emotional academic success factors improve (Aguayo et al., 2011). The issue of acculturation is significant for 
Raza students who are often faced with insufficient resources and obstacles in connecting with appropriate personal, academic, and social resources (Gloria et al., 2009).

According to Bordes and Arredondo (2005), a supportive environment for Raza students creates a positive outlook for them and increases the likelihood that they will persist with their education. Persistence in turn leads to the cultural adjustment that helps Raza regard the campus environment as positive. Participation in such an environment is recommended early in the college experience in order to provide Raza students with ample time to learn how to navigate the university system.

Raza students build spaces, often referred to as "counter spaces," based on cultural strengths that emphasize the need to be ambitious, hopeful, and connected to family in order to navigate the various cultural domains that a Raza student encounters as they balance multiple cultural elements (Ojeda, Navarro, \& Morales, 2011). Support systems that are culturally sensitive to the Raza population can contribute to safe counterspaces for Raza to become resilient and effectively navigate their university experience while remaining connected to their home culture and family (Yosso et al., 2009). Similarly, Solorzano's et al. (2005) analysis of the educational and racial barriers encountered by Raza college students found that a student's resiliency is predicated on their ability to take the cultural knowledge and skills from their home culture and apply it to create a welcoming university academic environment. Cerezo et al. (2012) also found that peers of the same ethnicity contribute to the cultural adaptation of Mexican Americans (MAs) by creating safe "counter-spaces" - through clubs, organizations, and 
fraternities/sororities - where MAs are able to develop the skills needed to contribute to a democratically engaged citizenry. The peers helped MA's by simply providing a model to navigate the university environment, helping younger or less-seasoned MA college students make connections about overcoming barriers and ways to excel in the university. These supportive experiences led to positive perceptions about what MA's are able to overcome, a direct result of MA's witnessing other MA's overcoming challenges first hand.

At the institutional level, counter-spaces are made possible by staff and leadership that promote a culturally sensitive curriculum, pedagogy, and culture (Yosso et al., 2009). Such institutions foster programs that aim to increase the number of graduating students of color, as well as a mission or vision that has cultural diversity as one of its pillars (Yosso et al., 2009). In response to Tinto's research on integration, many university institutions have instituted support services that assist students with cultural and social integration issues, navigating educational and career pathways, financial assistance, and developing healthy relationships (Saenz \& Ponjuan, 2009).

These counter-spaces have allowed students to gain mentors, a support system that has been tied to an increase in college completion as students are able to make more informed choices as a result of the formal and intentional mentoring relationship (BordesEdgar, Arredondo, Kurpius, \& Rund, 2011). For example, in a comprehensive study that explored the connection between student mentorship and the comfort levels of 112 first year Raza students, Bordes and Arredondo (2005) found that Raza students who 
participated in mentoring services reported improved acclimation to the university environment. Mentoring improved Raza students' determination to do well in school because they felt accepted as a result of mentoring relationships. Through their roles as academic advisors, friends, and colleagues, mentors help Raza students understand campus culture, providing the opportunity to tap into networks to help Raza students reach their academic and professional goals.

While the significance of peer support systems for Raza students is evident, the concept of "recognition" seems applicable as students in the studies cited above benefited from the legitimization process that took place during interactions in the various counterspaces. Rodriguez (2012) highlights a pedagogy of "recognition" that includes relational (how youth are greeted), curricular (how their knowledge and experiences are affirmed), contextualizing (understanding out of school experiences), pedagogical (instruction as linked to student realities), and transformative (critical stance toward educational goals). These components emphasize the need for the institution to make a conscious effort to address the issue of inclusivity for the Raza population. Castillo's et al. (2006) quantitative study exploring how 180 Raza undergraduate students' ethnic identity and perceived university environment impacted their college persistence attitudes, found that campuses grounded in culturally sensitive values and beliefs have a positive impact on student persistence.

Institutions such as the one highlighted in this study, with over one third of its Raza student population coming from the local area, would benefit from helping students 
bridge the resources that they have at their disposal as commuter students. Specifically, commuter students are able to maintain social and cultural networks as a result of being close to home. Additionally, financial and social mobility are motivating factors for Raza to persist in college (Schwartz et al., 2009). While it may be no surprise that economic status has a direct impact on GPA, Aguayo's et al. (2011) study measuring self-efficacy and academic performance found that Raza student perceptions of the financial resources available to them played a key role in their ability to persist. Indeed, when students have positive perceptions of finances, they are able to gain confidence in their academic performance (Aguayo et al., 2011).

\section{Raza Barriers to Degree Attainment}

Raza students who want to utilize support systems to benefit their educational experience still face significant barriers to earning their degrees. Currently, students outside the culture of power must put more effort into adapting to an institution that is unwilling to modify policies and practices that impact the students' ability to effectively navigate the various institutional domains (Kuh \& Love, 2000).

Raza students who are in Science, Technology, Engineering, and Math (STEM) majors face additional challenges. When tracking the degree attainment of Raza STEM students from 2004-2009, only 16\% graduated with a STEM degree in the six-year timeframe (U.S. Department of Education, 2015). Additionally, it is important to note that Raza students within an HSI institution face additional challenges. Just because a 
student is attending an HSI does not mean that Raza students are able to find the necessary support systems. Ojeda, Flores, and Navarro (2011) found that the institutional culture at the HSI institution that they studied was not conducive to the success of the Raza student population. In addition, their research found that Mexican students were facing unique challenges as a result of not having a strong connection to Mexican heritage, limiting their ability to make connections with other Raza students who had more of a connection. This challenge is part of a larger campus climate issue that leads Raza students to feel a sense of cultural intolerance from the dominant group, at times leading the student to engage in activities that may lead to institutional abandonment (Oseguera, Locks, \& Vega, 2009). At the university level, studies have found that interpersonal micro aggressions are encountered by Raza students through various interactions with other students, faculty, staff, and administrators. After racial macroaggression experiences, students may encounter feelings of withdrawal, self-doubt, and despair due to the fact that they internalize the negative stereotypes and comments they have received (Yosso et al., 2009).

The stereotypes and negative comments that students internalize as a result of racial micro agressions are some of the primary reasons why Raza students do not complete college (Castillo et al., 2006). Typically, students with a stronger ethnic identity encounter higher instances of discrimination due to their own awareness of the stereotypes associated with their ethnic and cultural group. This is a significant reality since students with a strong ethnic identity typically have a harder time integrating into 
the institutional culture. These stereotypes, along with the pressure caused by media portrayals of Raza populations, have led to excessive pressure and stress for Raza college students, ultimately leading to feelings of alienation within the institutional climate (Schwartz et al., 2009).

While "familia" can be a motivator for Raza student persistence, familial roles and expectations can also lead to stress (Ojeda et al., 2011). The pressure of living up to family obligations often takes a toll on Raza students. They and their families know little about financial aid and they are usually hesitant to take out loans due to a lack of knowledge about how loans work. Their lack of willingness to take out loans often leads to an increased financial burden that impacts their academic focus and their ability to persist (Cerezo et al., 2012). Further, the families' lack of knowledge of the university environment and the barriers their children encounter, also impacts students' ability to overcome the academic and social conditions they face. This lack of knowledge also leads many Raza families to encourage their children to look for steady jobs that do not require a college education and that in the short term, may lead to immediate financial gains (Cerezo et al., 2012).

Since Raza men are seen as the family providers responsible for the emotional and financial well-being of the family, Raza male students often face additional pressures from both their institutional environment and their family. Instead of reaching out for assistance to overcome the many problems that arise as a result of their role, many Raza males do not seek help due to social and cultural norms that expect the Raza male to 
solve problems on his own (Gloria et al., 2009). When students do not view staff as supportive, they become self-dependent in their coping efforts, an especially troubling trend for students who do not have the skills or knowledge to successfully navigate their undergraduate education. Ultimately, Raza male students who encounter these situations miss out on social and cultural capital building experiences that lead to positive relationships and student success (Strayhorn, 2010). Indeed, the persistence of Raza males is negatively impacted by a lack of participation in the college social environment that would help nurture Raza male students who are dealing with feelings of alienation (Figueroa, 2002).

Further, since the Raza culture values a sense of togetherness and collective responsibility, Raza male college students who do not participate in college support groups that help build a sense of family, may thus be unable to overcome the social, cultural, and structural challenges they face along their educational pathway. Overall, research related to gender roles has found that men across the board are not motivated to graduate due to the low return in investment they view in education (Diprete \& Buchmann, 2006). While research has identified the father as being responsible for helping the son develop academic and career ambitions (Buchmann \& Diprete, 2006), many Raza fathers are limited in their helping ability due to their unfamiliarity with the $21^{\text {st }}$ century educational and professional culture. Indeed, For Raza males who have adopted a "machismo" role, higher education can be viewed as feminine due to reflecting a "weak" or non-masculine culture. 


\section{Hispanic Serving Institutions (HSIs)}

Hispanic Serving Institutions (HSIs) are defined as "accredited and degree granting public or private nonprofit institutions of higher education with $25 \%$ or more total undergraduate Hispanic full-time equivalent enrollment (FTE)" (Santiago et al., 2015, p. 26). The HSI was officially declared in 1992 when then President George W. Bush signed HSI amendments to Title III of the Higher Education Act (HEA) of 1965 (Galdeano, Flores, \& Moder, 2012). For institutions to qualify for federal funds that are allocated for HSIs, they must apply for five year federal grants through the submission of a proposal that outlines strategies and practices that will have a positive impact on the Raza students at the HSI (Hurtado \& Ruiz, 2012). As of 2012-2013, there were 370 designated HSIs in the United States and incorporated areas (Santiago et al., 2015). Those 370 HSIs account for only $11 \%$ of higher education institutions but serve $59 \%$ of all Raza undergraduates in the United States (Malcom-Piqueux \& Bensimon, 2015). Based on those figures, HSIs are one of the lowest funded categories of institutions when considering the designation is meant to serve one of the most underrepresented groups in the United States (Galdeano et al., 2012).

Although a federal HSI definition is in place, the practical HSI definition will vary by institution (Galdeano et al., 2012). Very few studies have focused on examining the changes that are required for institutions to become truly "Hispanic Serving" (Hurtado \& Ruiz, 2012), a term that Malcom-Piqueux and Bensimon (2015) argues requires that institutions focus on improving academic success, not simply providing 
access to a higher education. According to Malcom-Piqueux and Bensimon (2015), recommendations for moving in this direction include: disaggregating educational outcomes data by race and ethnicity, ensuring faculty, staff, and administrators have the opportunity to reflect on how their pedagogical approach contributes to and/or hinders the academic development of students; ensuring that institutional components embody the HSI identity; and a collective approach to addressing the campus racial climate concerns In current HSI literature, Hurtado and Ruiz (2012) found inconsistencies in how HSIs operate and the specific initiatives that are contributing to the advancement of Raza in higher education. These inconsistencies may be the result of an unclear understanding of the meaning of HSIs within the context of individual institutions as well as gaps in the assessment of Raza student performance. Nunez, Sparks, and Hernandez (2011) highlighted Raza student traits that are unique to HSIs and often lead to greater challenges for students: higher percentage of low-income (43.6\% vs. 35.9\% for Raza at non-HSIs) and first-generation Raza students; higher percentage of Raza students who work full-time (41\% vs. $30 \%$ for Raza at non-HSIs); lower graduation rates for Raza students; and higher risk factors such as familial and financial obligations. On the contrary, there is research that supports the claim that Raza students at HSIs benefit from attending an institution with a larger Raza population - Cuellar (2012) found that Raza students at HSIs have more positive beliefs of self as compared to Raza students at nonHSIs

Malcom-Piqueux and Bensimon (2015) recently laid out a number of questions 
that should be considered when examining the effectiveness of HSIs: Why are our resources not reaching Raza students? How do Raza experience the resources offered? Are the resources we offer responsive to the actual needs of Raza students? What might prevent students from taking advantage of resources? While my study did not adopt those questions verbatim, both the research and interview questions aim to contribute to the discussion on institutional resources, the impact these resources have on Raza students, and recommendations for improvement. According to Bensimon, it is necessary to answer these questions in order to make the HSI designation more relevant to students and to ensure that institutional leaders are actively discussing what it genuinely means to be "Hispanic-Serving" (Malcom-Piqueux \& Bensimon, 2015). They further explains that in some cases, disparate outcomes for Raza students are unintentionally encouraged by institutions who believe individual student flaws are to blame for their lack of academic progress, instead of looking at how institutional systems are contributing to the challenges faced by Raza students.

To be truly equity-minded, institutions need to focus on improving practices that contribute to student success. A key component of this examination requires that faculty, staff, and administrators familiarize themselves with the life experiences and history of the Raza students that attend their institution in order to appropriately tailor resources that can help the students' development as scholars and as contributors to society (MalcomPiqueux \& Bensimon, 2015). This approach will have benefits for all students on campus, which is ultimately one of the goals of HSI funding - even if funding is awarded to 
institutions based on Raza enrollment, all students will benefit from the examination and cultural shift that occurs when institutions live up to the HSI label (Malcom-Piqueux \& Bensimon, 2015). Hurtado and Ruiz (2012) support this notion by emphasizing the need for HSIs to develop the culture and climate of the institution. The proposed institutional model would be reflective of the "Multi-Cultural Stage" that is highlighted in Hurtado and Ruiz (2012) - when institutions are in this Multi-cultural phase, there is consent amongst a variety of campus units about the need to establish clear goals that measure student success and address the needs of underrepresented student populations.

\section{Conclusions and Implications}

The above literature review has provided some background on the issue of Raza degree attainment, effective support systems, and the role of heterogeneity on Raza student academic performance. In regards to differences among multi-generational groups, the issue of Raza generational groups outperforming each other (GonzalezBarrera \& Lopez, 2013; Aguayo et al., 2011; Owens \& Lynch, 2012) is significant, but what most is concerning is the low degree attainment rates overall for Raza populations, regardless of generational status. The flow of Raza immigrants is constantly changing and it is very likely that changes in immigration policy will lead to a drop in college aged $1^{\text {st }}$ generation Raza and a significant increase in $2^{\text {nd }}$ and $3^{\text {rd }}$ generation (Gonzalez-Barrera \& Lopez, 2013). Since the above mentioned studies highlight the struggles of $2^{\text {nd }}$ and $3^{\text {rd }}$ generation Raza due to issues of discrimination, micro aggressions, and an overall lack of 
optimism, additional research is necessary that examines support systems that are effective for these populations, specifically at a Central California HSI.

It is also important to note that while research has highlighted the benefit of counter spaces, I believe these counter spaces are not always connected to other support systems that may be effective for multigenerational Raza, especially if counter spaces involve minimal interactions with administrators, faculty, and staff that do not reflect the ethnicity and/or culture of students in the counter space. There may be repercussions as a result of minimal interactions with administrators, faculty and students of the dominant culture, especially when Raza students come into interactions already on the defensive, fearing that they will be looked down upon or discriminated against.

There is a need to explore the benefits of positive interactions with administrators, faculty, and staff of the dominant culture, particularly as those interactions relate to effective support systems for Raza students at an HSI. Participating in the college social environment is necessary, but is helpful to Raza student achievement only when the interactions reinforce positive stereotypes. Further, since research has highlighted the challenges encountered by students who are not connected to their Mexican heritage, there is a need to examine the challenges associated with students being "too connected" to their heritage, and the impact that may have on students' ability to seek support. Specifically, there is a need for additional research on degree attainment contributing factors for HSIs specifically - how a "pedagogy of recognition," which includes relational, curricular, contextual, pedagogical, and transformative factors (Rodriguez, 
2012) - looks at an HSI.

Overall this research study addresses these questions in an effort to provide additional perspective on effective support systems for a multi-generational Raza college student population. As the diversity of the U.S. Raza population continues to increase, it is necessary to take a micro-level approach to addressing the degree attainment challenges of a large percentage of the U.S. population. 
Chapter Three: Methodology

\section{Introduction}

A qualitative research design was adopted in this study to examine the following questions: 1) How do family influence and the life and educational experiences of a multi-generational Raza student population across genders impact students' perception of support? 2) How does a multi-generational Raza student population across genders perceive the effectiveness of existing institutional supports at an HSI? 3) What additional supports do multi-generational Raza students across genders identify as necessary to succeed at an HSI?

The research was significant because it focused on exploring the unique experiences and academic needs of the multi-generation Raza population at Central Coast University (CCU), a 4-year HSI. The research shed light on the need to examine the role of support systems in addressing college degree attainment issues for a multi-generational Raza student population. Past research has called for the need to further examine specific Raza subgroups in order to learn how their culture-specific contexts impact their development (Gloria et al., 2009). Additionally, Reason (2009) found there is a need for more research focusing on specific demographic groups due to the fact that individual student attributes and socio-demographic components may impact their academic experience.

This chapter will first explain the study design and the process for the qualitative data collection. The role of the researcher will then be detailed, in addition to the process 
and criteria for participant selection, as well as the ethical approach I took to protect the human volunteers who participated as interviewees. The chapter will close with details regarding the data collection and data analysis procedures.

\section{Design}

A qualitative approach was adopted for this research due to the method's appropriateness to help me understand the student experience as it relates to the problem being studied (Silverman, 2010). A qualitative methodology is relevant due to the need to measure the impact and significance of student interactions with institutional components, an item that Kuh \& Love (2000) argued is difficult to measure through quantitative methodology. Additionally, the qualitative method allowed for an emotionalist approach to understanding the problem, focused on capturing authentic data that ensures contextual sensitivity, which means I am not basing my findings solely on my own assumptions and that I kept in mind various factors and context when collecting, analyzing, and reporting findings (Silverman, 2010).

This study applied a qualitative research design (Creswell, 2009) that was informed by a social constructivist worldview and a grounded theory strategy of inquiry (Glaser \& Strauss, 1967). A social constructivist worldview allowed me to analyze the data based on the worldview of the interviewee, as it relates to the issue being studied. In building on this approach, a grounded theory strategy of inquiry led to the development of a framework arising from the participant experience. Specifically, the constructivist 
approach allowed me to acknowledge that Raza student knowledge was influenced and developed by their past experiences, leading to the active creation of meanings throughout the interview interactions. Further, an emotionalist lens allowed me to understand how Raza interview participants described their feelings, as they relate to institutional support systems.

The study consisted of in-depth interviews focused on examining the support systems that are of value for multi-generational Raza students at the 4-year HSI. The study relied on purposive sampling to identify interview participants. Specifically, the study followed a stratified purposeful sampling approach in order to highlight unique traits of a variety of Raza subgroups and also identify comparison areas amongst the sampled and interviewed group (Patton, 1990). Maximum variation was also implemented as a result of selecting participants who ranged in undergraduate major, hometown, generational status, gender, and preferred Raza identification. Purposive sampling was appropriate for this study due to the need to identify students who: fit the selection criteria ("Raza" identification); completed the initial survey (to identify students interested in participating in the study); have had experiences with support systems in the academic setting (focus of this study); and provide me with an opportunity to examine the study research questions (Creswell, 2009). Once an appropriate group of students was identified through the purposive sampling method, I utilized a stratified sampling approach to identify students across all three of the Raza generations of interest as well as 
an equal distribution of students by gender (male and female - no students identified a different gender category).

Data were collected in Summer 2015 and early in the Fall 15 semester to accommodate the availability of a broad Raza student population, specifically those who did not regularly check their email in the summer months. I took full responsibility for collecting, coding, and analyzing the data.

\section{Role of the Researcher}

As the lead researcher for this study, I was in a unique position to engage students, staff, faculty, and administrators in discussions about the academic success of students at the CCU. Specifically, conducting this study at my employer institution allowed me to tap into my professional network to identify appropriate students for the study. I must note that I also utilized the survey component of this study to identify appropriate students whom I have had no prior interactions with and who are unaware of my work on campus. Overall, I believe my professional relationships with various segments of the student population allowed me to have strong rapport with all interviewees. Further, I believe my rapport and my position as an institutional employee allowed me to ask critical questions that elicit honest answers aimed at improving student services and institutional culture.

I believe the interviews provided me with an opportunity to gain a rich and indepth experiential account of the multigenerational Raza student college experience 
(Fontana \& Frey, 2008). The influence of portraiture was present as the interview analysis focused on the multiple voices and specific story of each interviewee, in an effort to "tell the stories of people who do not have 'voice' in academia (Chapman, 2005a). Portraiture allowed me to take an artistic approach to the research, possibly including poetry and storytelling components as part of the data analysis. Since many of the interviewees do not have a voice in the scholarly community, this study aims to shed light on the experiences of cultural and community groups that are often not endorsed (Chapman, 2005a). A portraiture influenced methodology is aligned with Critical Race Theory's emphasis on including the perspectives of people of color in research and countering the deficient and debilitated narrative that has historically been associated with those groups (Ladson-Billings, 2000). Ultimately, I believed that a mutual relationship built on effective communication, respect, and cooperation would help the interviewee establish an environment where they could be genuine in their admissions (Dance, Gutierrez, \& Hermes, 2010).

Through my professional work I see students encounter challenges on a daily basis. Thus, I am strongly vested in exploring ways to improve the support systems at this particular institution as well as contributing to research on best practices for Raza student populations. I see myself as an advocate in this study, as I took an ethical position that was in favor of the individuals being studied (Fontana \& Frey, 2008). Similarly, I adopted components of the Participatory Action Research (PAR) frame that was outlined by Duncan-Andrade and Morrell (2008), as I followed up with interviewees about some 
of the challenges and frustrations they had expressed as part of the interview. I made sure to see these interviewees as people who were willing to be open and vulnerable with me. Due to my position of power and potential to be of assistance, I felt it was necessary to embody the support system that I intended to examine through this study. Further, following the first three steps of the PAR process (identify a problem, research the problem, and develop a collective plan of action) allowed for this study to develop solutions to address some of the authentic problems the collective of interviewees discussed throughout. While the $4^{\text {th }}$ and $5^{\text {th }}$ step of the PAR process (implement the collective plan of action, and evaluate or re-examine the issue) is not complete, I am hopeful that the recommendations that come from the study findings will align with the campus strategic planning initiatives that aim to improve retention, inclusivity, and campus climate.

\section{Significance of Research Questions and Sub-Questions}

Within the context of an HSI, the research questions of the present study are aligned with questions that were presented in a recent HSI policy Brief (Malcom-Piqueux \& Bensimon, 2015) that discussed HSI design principles for equity that can assist institutions in assessing how well they are serving Raza students. The questions in this policy brief were: "Why are our resources not reaching Raza students? How do Raza experience the resources offered? Are the resources we offer responsive to the actual needs of Raza students? What might prevent students from taking advantage of 
resources? While I did not ask these specific questions to students in my study, I believe the interview questions I did ask (Appendix A) focused on similar themes, specifically as they relate to the support services that multi-generational Raza students perceive as beneficial to their well-being and academic development, as well as how their pre-college life and educational experiences have impacted their perception of support. Further, additional interview questions touched on factors that may prevent multi-generational Raza students from utilizing services, desired services, the marketing/branding of services, and student perceptions of the role of generational status and gender on supports and academic achievement.

\section{Context}

I believe CCU is an ideal institution for this study for the following reasons. First, the CCU is considered an HSI as a result of a $36 \%$ Raza student population. In addition, CCU has a high percentage of students who come from traditionally underrepresented backgrounds, with $58 \%$ of the student population identifying as first-generation to attend college and $35 \%$ as low-income. Further, there is an overall even representation of students from the various geographic locations of California (Northern/Central/Southern), with a majority of students (35\%) hailing from three local counties. As CCU moves forward with enrollment growth plans over the next five years, it will be necessary to ensure that the growth in number of students coincides with growth in infrastructure and support services offered to students. Finally, the growth in enrollment will almost 
certainly lead to an increase in the Raza student population on campus - Raza student enrollment at CCU has increased $10 \%$ percent since 2009 - further highlighting the need to examine the support need of a student population that is now the majority group on campus.

\section{Participant Selection}

I initially gained access to participants via the institutional student records database, which included name and email contact information for identified students. These students were sent an email (Appendix B) asking that they complete a sevenquestion researcher created survey (Appendix C). For those students who completed the survey, a purposive purposeful sampling approach was adopted in order to ensure a representative interview sample. Before responding to the survey, students needed to read and agree with an informed consent statement. Of the 2,303 students who were sent the survey, 370 students responded, representing $16 \%$ of the identified Raza student population. The responses provide me with a better sense of the Raza student generational diversity at CCU. The table below (Table 1) provides a snapshot of the 370 students who responded to the survey: 
Table 1: Profile of Raza Student Population at CCU $(n=370)$

\begin{tabular}{|c|c|c|c|c|c|c|}
\hline & Male & Female & Other & & & \\
\hline \multirow[t]{2}{*}{ Gender } & $100(27 \%)$ & $267(72 \%)$ & $3(.8 \%)$ & & & \\
\hline & 1st Gen & 2nd Gen & 3rd Gen & $N / A$ & & \\
\hline \multirow[t]{2}{*}{ Generational Status } & $76(20 \%)$ & $220(59 \%)$ & $61(16 \%)$ & $13(3 \%)$ & & \\
\hline & Hispanic & $\begin{array}{l}\text { Mexican- } \\
\text { American } \\
\end{array}$ & Mexican & Latino & Chicano & Other \\
\hline Self-Identification & $146(39.4 \%)$ & $141(38 \%)$ & $8(2 \%)$ & $37(10 \%)$ & $22(6 \%)$ & $16(4 \%)$ \\
\hline
\end{tabular}

I then sent another email (Appendix D) to all respondents to ask if they would be interested in participating in an interview. In an effort to obtain a stratified sample of potential students to interview, I made sure there was a fair representation of students based on the generational status, gender, and their preferred Raza identification. While the distribution is not necessarily equal, every intention was made to ensure as equal a distribution as possible. For instance, I made an effort to gather additional students who categorized themselves as $3^{\text {rd }}$ generation and males (across all generations) as a result of those groups having the lowest representation when the survey results were first reviewed.

Once a representative sample of students was contacted for the interview and students subsequently expressed interest in participating, interviews were confirmed for 25 participants. They were sent an informed interview consent form (Appendix E) and 
were asked to carefully read the form. If the participant was willing to participate in the interview after reviewing the form, they signed the consent form and were asked to bring a copy of the signed form to the interview. I worked with participants to find a convenient time for them to conduct the interview in person or via Skype, if necessary.

\section{Population and Sample}

Students who were identified as "Hispanic/Latino" by institutional records were the primary population for this study. The population included 2,303 Raza students, representing $36 \%$ of the 6,368 total number of undergraduate students enrolled at CCU in Spring 2015. Data collected from a researcher-created seven-question survey (Appendix C) allowed me to identify a representative sample of 25 multi-generational Raza students (even distribution of gender and generational status) for the interview component of the study. These students included those who identified as first, second, or third generation, as well as students who identified as Raza immigrants. In other words, participants for this study were CCU students who reported that they, their parents, or one or more of their grandparents came to the United States from any state in Mexico or Central America. All participants were over age 18 and there was no upper age limit for participation.

Responses from the survey were analyzed to determine which students met the inclusion criteria for this study: first, second, or third generation Raza students. Once the initial survey data were analyzed, I emailed all 370 respondents to request their 
participation for a 30-45 minute interview. Seventy-four of the 370 responded. I then engaged in the process of identifying a representative sample of students to interview for the study. I made sure to have generational status and gender represented as evenly as possible.

I also intentionally contacted students from a variety of areas in California as part of my intent to diversify the sample. The 25 interviewees were selected based on the following criteria: the need for at least 5 students from each generational group (first, second, and third); student's home region (students from all CA regions - Central, Northern, and Southern California); gender representation (aiming for a 1 to 2 ratio of men to women, I over-selected men, as the male to female ratio of Raza at CCU is 1 to 3); college major (ensuring applicable CCU majors are represented); and selfidentification ("Chicano," "Mexican-American," "Hispanic," "Latino," and "other" were all included). Some selections were also a result of interview availability and timely response to the email. I also considered student involvement (or lack thereof) in oncampus activities/clubs when selecting students to include students with a variety of life and educational experiences (Guiffrida, 2006). Once the 25 interviewees were confirmed, additional interested students who were not selected were placed on a backup list in case any of the initial 25 students were unable to take part in an interview.

The table below provides a breakdown of the students who were selected to participate in the interview: 
Table 2: Breakdown of Study Participants $(n=25)$

\begin{tabular}{|c|c|c|c|c|c|c|}
\hline Pseudonym & Gender & Year & Seneratior & Hometown/Birthplace & Major & Hispanic Identification \\
\hline Mary & $F$ & 3rd & 1 & San Diego/ Tijuana, MX & Business & Mexican-American \\
\hline Carina & $F$ & 2nd & 1 & Salinas/Michoacan, $M X$ & Computer Science & Mexican \\
\hline Juanita & $\mathbf{F}$ & 5th & 1 & Salinas/Salinas & Liberal Arts & Chicana \\
\hline Amanda & $\mathbf{F}$ & 3rd & 1 & Watsonville/Guanajuato, $\mathrm{MX}$ & Psychology & Hispanic \\
\hline Lupe & $\mathbf{F}$ & 3rd & 1 & Salinas/Guanajuato, $\mathrm{MX}$ & Social Science & Latina \\
\hline Juan & $M$ & 2nd & 1 & Watsonville/Jalisco, MX & Business & Hispanic \\
\hline Jesus & $M$ & 5th & 1 & Long Beach/Long Beach & Liberal Arts & Mexican \\
\hline Daniela & F & 2nd & 2 & Anaheim/Garden Grove & Biology & Hispanic \\
\hline Evelyn & $F$ & 4th & 2 & Salinas/Salinas & Health & Latina \\
\hline Anna & $F$ & 2nd & 2 & Salinas/Salinas & Computer Science & Mexican-American \\
\hline Isabella & $F$ & 5th & 2 & Watsonville/Watsonville & Communication & Mexican-American \\
\hline Gina & $F$ & 4 th & 2 & Castroville/Watsonville & Liberal Arts & Mexican-American \\
\hline Alondra & F & 4th & 2 & Wasco/Bakersfield & Psychology & Chicana \\
\hline Sofia & $\mathbf{F}$ & 3rd & 2 & Salinas/Salinas & Psychology & Mexican-American \\
\hline Camila & $F$ & 4th & 2 & Salinas/Bellflower & Social Science & Latina \\
\hline Valerie & $F$ & 4 th & 2 & Los Angeles/Los Angeles & Social Science & Mexican-American \\
\hline Samuel & $M$ & 2nd & 2 & Rialto/Monterey Park & Biology & Mexican-American \\
\hline Joaquin & M & 4th & 2 & Salinas/Salinas & Business & Mexican-American \\
\hline Lucas & $M$ & 4th & 2 & Hollister/Gilroy & Graphic Design & Mexican-American \\
\hline Emiliano & $M$ & 5th & 2 & Salinas & Psychology & Hispanic \\
\hline Felipe & $M$ & 3rd & 3 & Lindsay/Stratmore & Social Science & Human \\
\hline Ximena & $\mathbf{F}$ & 4th & 3 & Tracy/Oakland & Biology & Hispanic \\
\hline Victoria & $\mathbf{F}$ & 4th & 3 & San Jose/Midland, TX & Psychology & Mexican-American \\
\hline Julieta & $\mathbf{F}$ & 4th & 3 & Fresno/Fresno & Psychology & Mexican-American \\
\hline Martin & $M$ & 5 th & 3 & Sacramento/Sacramento & Psychology & Latino \\
\hline
\end{tabular}

\section{Instrumentation}

For the survey component, all students identified as "Hispanic/Latino" in the database of CCU students for Spring 2015 received a direct email explaining this research study, the informed consent process, and were asked to complete a short survey that asks for their name, gender, email, generational status, self-identification (Chicano, Hispanic, Latino, Mexican-American, other) and college major (Appendix C). The interviews on the other hand, provided a rich and in-depth experiential account of the CCU college experience of multi-generational Raza students across genders (Fontana \&

Frey, 2008). 
Survey response data (gender, self-identification, generational status, identification, and student involvement) is presented in descriptive tables in the sections above to shed light on the heterogeneity of the Raza population at the 4-year HSI. All of the data included in the descriptive tables were examined in order for me to select a diverse sample of Raza multi-generational students to interview. Because this research was particularly focused on the experiences of $1^{\text {st }}, 2^{\text {nd }}$, and $3^{\text {rd }}$ generation students, the interviews targeted only Raza students who fall into those categories.

I believe the interview data sheds light on the impact of support systems on the multi-generation Raza population across genders at the 4-year HSI, specifically the role of support systems on academic persistence for the identified population. For data analysis of qualitative interview data, Grounded Theory (Glaser \& Strauss, 1967) and elements of Discourse Analysis (Wood \& Kroger, 2000) were applied. In addition, elemental coding methods (Saldana, 2012), specifically In-Vivo, Process, and Descriptive coding were implemented to assist in the development of themes from the interview data. Descriptive coding served as the initial coding method as I summarized phrases and passages that I believe were significant. This process took place while I was waiting to receive the transcriptions from the company I hired to transcribe. Listening back to the interviews allowed for me to spend some time feeling out and comprehending the words and passages of interviewees.

Once the transcriptions were received, I was able to engage both In-Vivo and Process coding methods (Saldana, 2012) to capture the exact words of interviewees (In- 
Vivo) while also capturing words or phrases that signified an action or a consequence (Process). All three coding methods were adopted to ensure that data validity and reliability concerns were addressed through multiple approaches to analyzing and coding the data. In-vivo specifically addressed data reliability issues as a result of the coding methodology providing a foundation for coding consistency (Silverman, 2010). It captured the rawness of the data, which helped minimize the shaping of the data by my pre-conceptions. Coding software was not utilized as part of the coding process. According to Saldana (2012), coding with the actual words of interviewees enhances and deepens understanding of their cultures and worldviews.

I maintained a journal to keep track of emerging themes as well initial thoughts that arose during the interviews. I was responsible for analyzing all transcriptions of interview data. Shifts between Spanish and English were noted and transcribed as spoken. Nonverbal communication was not factored into the transcription or data analysis. As the data analysis took place, I acknowledged there was a need to take into consideration the belief that words can have contrasting meanings in different conversational situations (Gee, 2004). This way of thinking led me to review the data in multiple ways to ensure that I had multiple opportunities to examine the data.

While I initially planned to transcribe all interviews myself, I had to change my approach due to time limitations. I contracted an online service to fully transcribe all 25 interviews, a process that took about one week to receive the transcribed interview documents. While I waited to receive the transcription in a MS Word document, I 
listened back to each of the interviews and develop a quick outline that included comments that stood out, ideas that came to mind as a result of their comments, and reflections about how the responses relate to the research questions of this study. Once this process was complete for all interviews, I compiled the notes and analysis into one document in an effort to develop an initial list of codes that I would then refer back after analyzing the transcribed documents. This data analysis process was informed by the Descriptive coding process outlined by Saldana (2012) that is based on summarizing the phrases, topics, or ideas of the interviewee.

After receiving the transcriptions, I reviewed each transcription individually and analyzed the data by Descriptive, Process, and In-Vivo coding (Saldana, 2012).

Descriptive coding provided me an opportunity to gain a higher level of intimacy with the audio interview recordings while I waited to receive transcriptions. The Process coding allowed me to look for actions or consequences in the statements shared by interviewees. In addition, In-Vivo coding allowed me to take the words/phrase from the interviewee as part of the code and theme development process. Once this process was complete for each interview, I began compiling the phrases and ideas across the interview sample in an effort to get a better sense of codes and themes that were most common for the collective. I kept this document organized by labeling different data sets with tags such as "Family," "Challenges," and "Support Systems," among others. I then followed the same data organization process for the document I created based on listening back to the interviews. 
The next step required that I compare the list of developing codes from the transcriptions with the list I had compiled after listening back to the interviews, a process that allowed me to review the data in multiple ways and on different occasions. The codes were then grouped into categories, and examined to explore connections across categories to create more complex themes. The organization of the data in outline format then allowed me to split themes based on the variables that were the focus of this study, specifically generational status and gender. I did consider integrating other variables but decided to focus on generational status and gender due to time limitations and not wanting to muddy the findings. The extensive process then led to the development of three interrelating themes that provided a representation of the underlying message behind the participant interview data: (1) family away from home, (2) sense of belonging, and (3) internalized oppression. The grounded theory approach to data analysis also allowed for the development of a conceptual model that provides a visual of the emerging data.

\section{Reliability and Validity}

As Torres (2003) mentioned in his study, examining the data and analysis with colleagues and interviewees contributes additional angles to view the data. In an effort to apply this recommendation, I asked the interviewees to review the analysis and to provide feedback in an effort to ensure accurate, trustworthy, and credible analysis and to "member check" the qualitative findings (Creswell, 2009). In addition, I shared interview 
analysis notes with a colleague in an effort to gain feedback and additional perspective on the data. Sharing the analysis with the interviewees, as well as colleagues addresses data validity concerns as a result of analysis that includes a variety of perspectives (Silverman, 2010). In an effort to address data reliability, specifically in regards to consistency in coding, I primarily applied In-Vivo coding methods in an effort to ensure there was minimal deviation in the definition of codes as well as the meaning of codes as the coding process took place (Creswell, 2009).

\section{Limitations}

The qualitative research design of this study provided a look into the perceptions of support among a multigenerational Raza student population across genders. The study utilized a grounded theory approach (Glaser \& Strauss, 1967) in an effort to allow for codes and themes to arise based on student responses. A qualitative approach was appropriate for this study as past research has found the methodology is appropriate for examining campus culture integration issues for students of color (Guffrida, 2006) and non-academic factors that impact persistence and retention (Keels, 2013). On the other hand, I acknowledge limitations as a result of how interview questions (Appendix A) were framed and the inherent bias in that approach (Silverman, 2010). Further, there are limitations with the study design as there could have been other variables that were accounted for in the analysis of the Raza student experience with supports, specifically social class, race, and grade point average, all of which could have provided a more clear 
understanding of the multiple factors that impact Raza students' perception of support. As a Mexican-American, I believe there are also limitations and bias involved with my approach and pre-conceived notions based on my own experience as a student at this institution a decade ago.

Limitations with the data set included a lack of response to the initial inquiry for students to complete the survey. Since there were no financial and/or other incentives, students may have been hesitant to participate. Students may have also not completed the initial survey due to their belief that the survey would take too long. An additional challenge involved finding a representative sample of students to interview. Considering the various Raza subgroups I proposed to interview, there was a need to engage in additional outreach for students who identified with the subgroups that lacked representation. In addition, immigrant students may have been hesitant to participate in an interview due to a fear of repercussion or of being labeled. Due to time limitations, nonverbal communication was not factored into the data analysis. The utilization of transcription symbols was also excluded from the data analysis due to the process being above my ability for this paper, although I do understand the significance of utilizing transcription symbols in discourse analysis. As a researcher trying to learn about others and the problems they face, I have also been able to identify things about myself (Fontana \& Frey, 2008). In this case, the data collection and analysis helped me identify gaps in my interview questions as well as my analysis of the data. Limitations were also present 
as a result of a methodology that made it difficult to understand the full impact of the intersectionality of generational status, gender, and perceptions of support.

There is also a possibility that my position of power led students to respond in a certain way as part of their efforts to please an authority figure by providing responses that they believed I wanted to hear. Other limitations may have been cultural in nature some Raza students may have a difficult time discussing cultural elements and their experience interacting with various institutional components. As limitations presented themselves, I discussed challenges with members of his dissertation committee, CCU Staff, Faculty, and Administrators (to encourage appropriate students to participate), and Ed.D cohort mates who provided valuable insight on how they were able to address challenges with accessing participants and/or data sources. 


\section{Chapter 4: Findings}

"You have to feel that those persons that are supposed to be offering you support can really understand you." - Juanita, $I^{\text {st }}$ Gen

Juanita's comment resonates throughout this chapter. For Juanita specifically, the need for support staff to understand may take on various forms and may be inclusive of a variety of academic, personal, and career development needs. While understanding the specific needs of Raza students like Juanita is important, this study to examined unique needs of a diverse Raza student population at this Central California four year public HSI. To understand these needs, the study focused on capturing the experiences of Raza students across gender and generational status. As previous chapters have noted, past studies have resulted in ambiguous findings in regards to the academic achievement of Raza students by generational status (Gonzalez-Barrera \& Lopez, 2013; Owens \& Lynch, 2012; Portes \& Rumbaut, 2001). As a result, this study focused on exploring the unique support needs for students at a California HSI based on their generational status and gender. Given that Raza males have the lowest percentage of educational attainment of the U.S. Raza population (Lee \& Ransom, 2011), it was necessary to also examine any differences in perceptions of support for Raza students based on gender.

This study used a qualitative research design, although data were collected in two phases, with the first using quantitative methods to identify demographic patterns of 
enrolled Raza students, including their generational status, which is not recorded by the university. This quantitative survey was sent to 2,303 identified Raza students at the institution. Of these students surveyed, 370 students (16\%) responded and a purposive sampling approach was adopted to identify a representative sample based on generation and gender to interview. Thus the second and most important phase of data collection involved individual semi-structured interviews of 25 Raza students who identify as $1^{\text {st }}$, $2^{\text {nd }}$, and $3^{\text {rd }}$ generation Raza. Each of them was asked a series of questions dealing with their perception of support, the support systems they desire, and the support seeking challenges or barriers they face at a 4 year HSI in Central California. Every intention was made to ensure males were adequately represented in this study, and 8 of the 25 students interviewed were male. This represents $32 \%$ of the interview sample, which is a fairly accurate reflection of the $37 \%$ male population at the institution for the $2015-16$ academic year. In addition, while the institution does not collect data on generational status, the students selected for interview reflect the generational breakdown of the students who answered the survey. $20 \%$ of students who answered the survey identified as first generation, $59 \%$ as second generation, and $16 \%$ as third generation. In turn, $28 \%$ of interviewees were first generation, $52 \%$ were second, and $20 \%$ were third.

Using grounded theory (Glaser \& Strauss, 1967; Saldana, 2009) I developed a framework springing directly from the participants' experiences. Findings are organized into three sections that discuss the themes that emerged from the analysis and that describe their connections with each other and to the research questions. The three 
themes that emerged are: (1) family away from home; (2) sense of belonging; and (3) internalized oppression. I developed a graphic model that shows the relationship among the three themes and to the left shows the inputs from home, generational status and gender, and education. The themes are integrated in the model below as part of the Raza College Student Support Model that was developed for this study:

\section{Conceptual Framework}

The diagram in Figure 1 provides a framework for understanding the support needed by a multigenerational Raza population across genders at an HSI institution. On the left are the inputs, which acknowledge the capital that students bring with them to the institution in addition to the capital gained during their educational experience at the institution. This capital is a result of family influences (these account, in part, for generational status and gender) and student experiences in the social and K-16 educational environment. While generational status and gender funnel into the family influence input, they are highlighted as inputs because of the study focus on examining the unique needs of Raza college students at an HSI. 


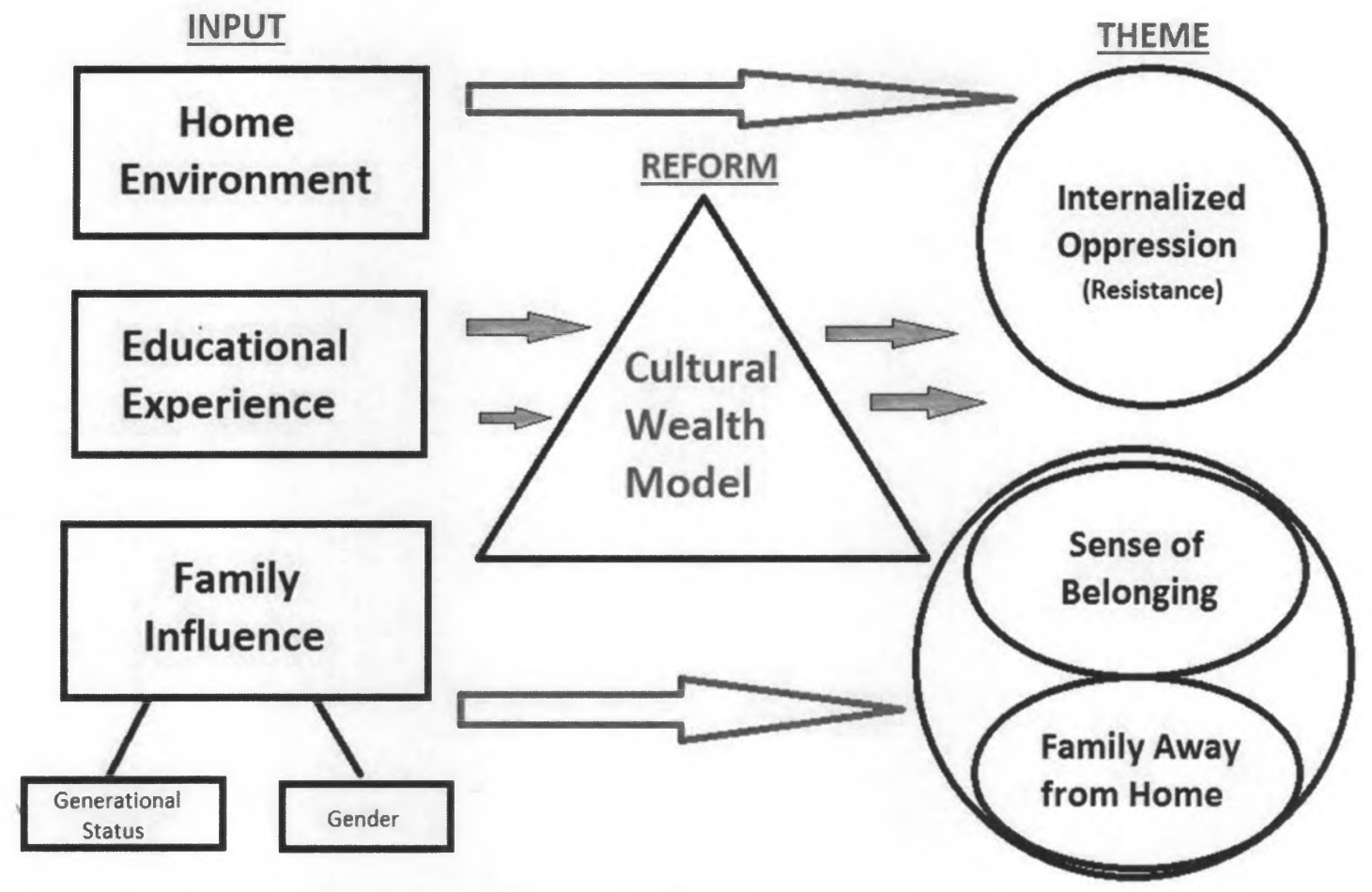

Figure 1: Raza College Student Support Model

The Cultural Wealth model (Yosso, 2005) sits in the middle of the diagram as a reform to address the needs of Raza students at the institution, due to my belief that the six types of capital included the model (aspirational, linguistic, familial, social capital, navigational, and resistance) must be accounted for by HSIs who are examining ways to improve the educational experience and academic outcomes of Raza students. Further, Yosso's model allows for an examination of the HSI identity of the institution, a necessary practice, as there is a need for additional HSI research and documentation of HSI best practices (Hurtado \& Ruiz, 2012). Yosso's (2005) model serves as both a mechanism for processing the experiences and capital students bring with them while 
also serving as a reform for addressing student needs that fall under aspirational, linguistic, familial, social capital, navigation, and resistance categories. The capital and experiences students bring with them are processed through Yosso's (2005) model whether they have the potential to serve as a positive or negative influence for the student in college, as indicated by the smaller arrows on both sides of the model. Any negative influences from the inputs are processed in an effort to identify ways Yosso's (2005) model can serve as solution while positive influences from the inputs are processed to ensure the institution is identifying these influences in an effort to then build on them to address any issues the student is experiencing in the realms of family environment, sense of belonging, and internalized oppression.

The model acknowledges that the capital students bring with them and the capital they develop during their time at the institution has both positive and negative outcomes, although findings from this study conclude that the capital students bring with them is for the most part an asset. Study themes are included on the right of the diagram, all of which are the result of student interactions with the institution and the influence of the inputs on the left side of the diagram. The themes include both negative (internalized oppression) and positive (family and sense of belonging) outcomes that can either be reinforced or addressed by educational institutions. The diagram assumes that when the Cultural Wealth model (Yosso, 2005) is implemented, Raza student sense of belonging, family identity, and components of internalized oppression and self-preservation can be 
addressed, resulting in a more positive educational experience and academic outcomes for those students.

\section{Research Question Significance}

One goal of this study is to gather a better understanding of the supports available to Raza students at a four-year public university in California, based on their testimonies. The school serves $37 \%$ Raza students and has had the HSI label since 2008. Currently, the staff, faculty, and administrators are exploring the significance of the HSI label at the institution, specifically how the HSI identity plays out and how the HSI label is or is not leading to improved educational outcomes and experiences for Raza students. The Raza population in California and the United States more broadly continues to expand, yet Raza are persistently underrepresented in their enrollment and graduation rates from fouryear institutions. Clearly, educational leaders must develop more effective reforms.

The study asks three research questions. The first question is: How do family influence and the life and educational experiences of a multi-generational Raza student population across genders impact the students' perception of support? I asked interviewees a variety of questions relating to their perception of support at the institution, as well as their perception based on their life, educational experiences, and familial influences. Overall, this first question sought to understand the academic, social, and cultural resources students brought with them to the institution and what they discovered once they had enrolled. 
The second research question asked how a multi-generational Raza student population across genders perceived the effectiveness of existing institutional supports. This part of the study sought to evaluate the quality of resources that this institution provides to the Raza students who are attending and to understand if there are any differences in how these services are used based on gender or generational status.

The third research question looked to the future asking students what additional supports they need to succeed. Again the study design was intended to uncover any differences by generational status or gender. I asked questions relating to the ideal educational environment that interviewees believe would maximize their chance for academic success at the HSI.

It was important to frame the research in terms of these three sub-questions because each focuses on different components of support, including the capital students bring with them to school and how that capital plays out as they interface with support services at the university (question one), as they evaluated the effectiveness of support services they accessed or chose not to access at the institution (question two), and as they reflected upon unmet needs for support as they continue in their educational journey at the institution (question three). It was also important to design the study to allow a comparative focus on generational status because past research has resulted in ambiguous findings on the impact of generational status on the college experience (Gonzalez-Barrera \& Lopez, 2013; Aguayo et al., 2011; Owens \& Lynch, 2012). Finally, the study design needed to provide a way to drill deeper in a comparison of student experience by gender 
due to the low degree attainment rates of Raza males (Lee \& Ransom, 2011), as well as the need for additional research that explores reasons for males' lack of participation in college support groups (Figueroa, 2002). It was also important to identify reasons why more Raza females are taking advantage of the support groups on campus as well as the challenges the women encounter in seeking support.

Finally, the study design is significant as it focuses on a single institution with a Raza student population that continues to grow - from $27 \%$ in 2009 to $37 \%$ in 2015 . The percentage of Raza students will continue to increase as the institution moves forward with plans to grow to 12,500 students by 2020 , a $44 \%$ growth from the current enrollment of 7,000 students. While there are commonalities and differences in regards to what support Raza students need at this institution, it is important to understand distinct supports may be required depending gender and generational status.

\section{Three Major Themes}

In the sections below, I describe my findings based on the three themes. The first theme, "family away from home" focuses on where students are coming from as well as the knowledge and experiences they bring with them, both of which have negative and positive outcomes. The second theme "sense of belonging" focuses on practices and strategies that students describe as conducive to positive environments for support and help students address barriers that limit their ability to utilize and benefit from support systems. The third theme, "internalized oppression" is troubling and came to light as 
students described negative experiences before arriving at the institution as well as instances at the current institution. Overall, the three themes are representative of the experiences of students who were interviewed for this study and highlight the need for HSIs to examine multiple components to identify ways to improve educational outcomes for a multigenerational Raza population across genders.

The following sections are organized by the three themes in the following order: "family away from home," "sense of belonging," and "internalized oppression." Each of the sections begins with a quote that I believe is representative of the theme itself. In addition, quotes that are italicized before the beginning of a new paragraph represent the beginning of discussion on one of the sub-themes (Table 3) that make up each of the three themes. When necessary, I provide additional analysis about how student responses connect back to the study themes, sub-themes and research questions, with specific attention paid to the role of generational status and gender in student responses. The chapter concludes with a discussion on how the HSI Student Support conceptual model played out throughout the findings as well as a comparative analysis of the study themes, the connections and tensions across the themes, and next steps 
Table 3: Study Themes and Sub-Themes

\begin{tabular}{|l||c||c||l}
\hline THEME & Family Away From Home & Sense of Belonging & Internalized Oppression \\
\hline SUB-THEMES & Confianza & $\begin{array}{c}\text { Support During } \\
\text { Transition }\end{array}$ & $\begin{array}{l}\text { Challenges in Reaching } \\
\text { Out }\end{array}$ \\
\hline & $\begin{array}{c}\text { Mirroring the Home } \\
\text { Support System }\end{array}$ & Cohort as Family & $\begin{array}{l}\text { Fear of "Discrete" } \\
\text { Implications }\end{array}$ \\
\hline Home Challenges & Finding a Connection & $\begin{array}{l}\text { Impact of Pre-College } \\
\text { Experiences }\end{array}$ \\
\hline & $\begin{array}{c}\text { The Commuter Experience } \\
\text { Gender \& Generational } \\
\text { Factors }\end{array}$ & $\begin{array}{c}\text { Sarketing/Branding of } \\
\text { Services }\end{array}$ & Hood Mentality \\
\hline
\end{tabular}

\section{FAMILY AWAY FROM HOME}

I have some family members that, they didn't end up going to school and I look at them and I say, "How come they didn't go the same way that I did?" I think it's because of my parents. I look at their struggles and I say, "I don't want to end up that way."

$$
- \text { Juan }\left(1^{\text {st }}\right. \text { Gen) }
$$

This quote from Juan highlights the importance of familial support for all but two of the students interviewed for this study - all students discussed elements of family to some extent although only two specifically focused on the lack of familial support. Juan and many other interviewees pointed to the support provided by their parents and other family members who serve as mentors, motivators, and guides. The support that students receive from their family takes on various forms and thus it is necessary to gain an 
understanding of how family support experiences play out in the multigenerational Raza college student experience. For Mary ( $\left.1^{\text {st }} \mathrm{Gen}\right)$, her family served as a cheer team, constantly reminding her that she is capable of being successful. In addition, her parents also posed various questions that took an active approach to identifying the challenges she encountered. She said, "he asked me about my well-being as well like, "Are you little stressed?" like, "What's going on?" The cheering and questioning approach taken by the student's parents made her feel well supported, which in turn made her feel she had a duty to make her parents proud, specifically as a $1^{\text {st }}$ generation Mexican-American who was born in Mexico and didn't come to the United States until $6^{\text {th }}$ grade.

Aside from parental support, Juan $\left(1^{\text {st }} \mathrm{Gen}\right)$ highlighted the significance of having a brother who served as a mentor and guide, specifically encouraging him to utilize support systems on campus. Juan said, "I would feel like, 'I don't want to ask for help because I'm going to feel different.' He [brother] says what are you going to [do] -- it's only going to help you, it's not going to put you down or anything..." What did Juan mean by feeling "different?" His sense of "feeling" different is described negatively. There seems to be a sense of exposing himself to someone he doesn't know and the fear and challenge that comes with that. This fear becomes an obstacle, which is encountered by students like Juan who feel different and highlights the needs for these students to develop a family network as they transition to the university. Although Juan felt uncomfortable at college and didn't want to ask for help, his brother helped him see the resources that are available and helped to normalize the experience of accessing these 
supports. Such a network may serve to provide students with access to a variety of supports that can then have a positive impact on the students' various areas of development.

The significance of a family/team as students transition to college was discussed by many interviewees, specifically the impact that family/team has on a student's ability to build rapport with peers and campus staff. While Juan benefited from a brother who had access to college culture, other students like Carina ( $\left(1^{\text {st }}\right.$ Gen $)$ did not mention any benefits from having had an older sister who graduated from college. Carina stated her sister graduated with a 4-year degree, but also said that her sister had experienced trouble navigating elements of the college system. This reality possibly led her to have reservations about the value of the support, guidance, and words of encouragement she could provide to Carina. Both Carina and Juan are first generation Raza students who had an older sibling who graduated from college, yet they had opposite experiences. Thus, having an older sibling or cousin who has attended, or even graduated from a four-year institution does not guarantee that students will have social capital from home that allows them to feel confident asking for help from the university.

\section{Confianza and Mirroring the Home Support System}

The rapport that students develop with peers and campus staff leads to a sense of "Confianza," a term that was used by one student to describe her need for family-type support system: "I can go personal in anything that I have, I kind of feel more, in 
confianza," - Gina ( $2^{\text {nd }}$ Gen $)$. For this student, the confianza needs to be established before she feels like she can "go personal," a statement I interpret to mean she is willing to open up about non-academic challenges she may be dealing with. Similarly, Amanda $\left(1^{\text {st }}\right.$ gen) discussed the need for patience as it relates to helping eliminate the fear that some students may have about interactions with institutional staff and faculty. This patience is also directly tied to the warm personality and confianza that students mentioned as important. This sense of confianza and its role in both a personal and academic support system is summarized by Evelyn $\left(2^{\text {nd }}\right.$ Gen $)$, who mentioned how an effective support system for her is one that mirrors the support provided by her family:

And I feel like my parents have always been it, they would always want the best for me so I feel like the advisors kind of like do the same; they always want the best for their students. They just keep you going. They talk to you through it... whatever it might be. - Evelyn $\left(2^{\text {nd }} \mathrm{Gen}\right)$

Evelyn highlights the significance of supports that mirror the system established by her parents, a reality that highlights the need for support staff to get a better sense of a student's life experience before identifying the most effective way to engage the student. While Gina (2nd Gen) does not say the development of rapport with support staff needs to mirror the support provided by her family, Gina overwhelmingly spoke positively about her family support system, leading me to assume there is a direct connection between the development of confianza at home and at the institution. For first generation students Juan and Carina, neither discussed the significance of the family support system 
although they did provide examples of how family has benefited them in different ways: Juan referenced the support provided by his brother, while Carina mentioned the significance of family gatherings as a way to de-stress. It is important to understand the significance of family dynamics as students encounter support systems at the college level. The examples provided above to support this claim were representative of interviewees who are first and second generation Raza students and with the exception of Juan, all females.

Male and third generation interviewees also discussed elements of a family/team environment, but their comments related to family support were either more likely to emphasize challenges (was the case for third generation female Ximena and second generation male Samuel) or lacking detail, as was the case for about half of the males interviewed for this study. or Two of the first generation males Juan, discussed above, and Julian who will be discussed later, described family support in more detail, possibly because their first generation status led them to perceive a closer connection to their home culture. Second generation male students Emiliano and Felipe also discussed some of the benefits of their family support, although they spoke more about the challenges they faced as a result of navigating their family support system.

Similarly, first generation Juanita highlighted the importance of faculty and staff members at an HSI to really understand the support system a student had at home, not only to find out what is working for them, but also to identify the challenges and obstacles that are created. Juanita said: 
...I've been realizing is those stark preferences between how they [parents] were raised and how I'm raised. I think that's where that clash comes in and I definitely know that it's not that they don't want me to be successful. It's just we come from way different places... - Juanita (1st Gen)

\section{Home Challenges}

In some cases, Raza students across generations and genders encounter a sense of support from family that at times may lead to additional difficulties as the student learns to adjust to a new university environment. Gina $\left(2^{\text {nd }} \mathrm{Gen}\right)$ mentioned the difficult balance that comes from living at home while attending college - there is an expectation that she will continue to take an active role in well-being of family members (helping clean, cook, chores, etc.) while maintaining high grades. While this student mentions this as "hard to balance," she acknowledges this reality as coming with the territory of living at home. Other students like Anna ( $2^{\text {nd }}$ Gen) also discussed the challenges that come from dealing with family, albeit in a more somber tone as she is lacking encouragement, praise and positive feedback from her family.

I wish my I could say my FAMILY [is supportive] but I feel it's not enough, at least not for a healthy well-being, I feel like they're doing more HURT than actually helping me but it's not really much. - Anna (2nd Gen)

Anna acknowledges that her family makes an effort to be supportive but that is not enough to have a positive impact on her. Only one other student mentioned the 
challenges associated with a lack of a parental support, once again highlighting the fact that parents make an effort to support, but are often limited due to various factors:

My family doesn't really help as much because they're not really supportive people. But they help me out when they really can at times... My dad's always been like distant. He's been the financial support with the family... My mom, she was very, like she puts herself in front of us at times and that's just like, I feel really disrespected, when she does that especially now. - Samuel (2nd Gen)

For Samuel, a second generation male, the lack of support highlights both cultural and educational factors that limit his parents' ability to provide support. On one end, he views his father as solely a financial support, while on the other hand, he sees his mother struggling with her own personal challenges, which limits her availability to Samuel. Another student brought up a unique "help seeking" limitation based on how much she had depended on her father in her early years of schooling. She said,

I would always just rely on like, 'My dad will help me at home.' But then once I got to high school, it was like, 'He can't help me anymore.' But I just was so immune to asking for help, because I was used to, like help from my family...-Alondra ( $2^{\text {nd }}$ Gen)

For Alondra specifically, a strong reliance on her father led to a sense of dependence on her father for help, leading to a lack of understanding about what resources and support she needed to be successful in school. In addition, Alondra referenced a negative experience with a white teacher that led to additional help-seeking reservations as she continued her schooling (that example is referenced later in this chapter). For students 
who discussed challenges associated with the support provided by their parents, none of them expressed themselves in a way that made it sound like the lack of support and additional challenges as a result of the parent approach were intentionally trying to hurt the interviewee. On the contrary, the students highlighted a sense of cultural disconnect that is evident as they navigate the support seeking systems established by their family and those established by the educational institutions they encounter. The cultural disconnect was also evident with Felipe ( $\left.2^{\text {nd }} \mathrm{Gen}\right)$, who described his parent's lack of understanding as to why he wants to continue his education past undergrad, an issue that he admits makes the situation more difficult as he thinks about the type of support that will be available to him if he does decide to continue his education:

When I did tell them, 'I want to go all the way to a doctor.' They go, 'How many more years?' I tell them, 'Just eight, maybe nine.' They're like, 'Really?' I mean, yeah, really. They're like, 'What are you going to do, are you going to work? What's going to happen? How you're going to pay for it?' - Felipe (2nd Gen)

For other $3^{\text {rd }}$ generation students like Ximena, the generational status did not necessarily have an impact on her family's perception of education. Ximena ( $\left.3^{\text {rd }} \mathrm{Gen}\right)$ admits that education was still very low on the priority list in her family. She expressed a unique challenge involving the tension she feels from her parents as a result of trying to be, as they perceive, "better than them":

I think that it was hard trying to tell my parents, 'I want to be better that you,' and I said that in quotes because it is like that's the way my parents perceive it. They think that, at least for my mom I know that it was a 
tough transition for her because I want to be something higher than her career. It's like [my mom feels like], 'Why does she want to be better than me?' - Ximena ( $3^{\text {rd }}$ Gen $)$

It is important to note that a number of comments in regards to challenges with parent support systems were made by $2^{\text {nd }}$ generation female students, a majority of whom are commuting to college and are living at home with a parent or other close family member. Unique family support concerns were also discussed by male student Felipe and $3^{\text {rd }}$ Gen student Ximena, albeit the challenges they discussed were different - Felipe's comment highlights the lack of family understanding for continuing education while Ximena's quote highlights a sense of parents feeling threatened as a result of the educational/career aspirations of their children.

\section{The Commuter Experience}

For commuter students specifically, there was a collective call for a more structured support system, as these students who live off campus often find it difficult to create a community of support at college. The disconnect due to their physical separation from campus means that there is also a philosophical separation that takes place as students are disconnected from elements of campus life that may be beneficial to them. In contrast, a number of commuter students expressed their satisfaction with the quiet study spaces in the library, since many of them do not have a quiet space to study at home. Or even if they did, they are often expected to assist with household duties that 
take valuable time away from their studies. As Juanita (1st gen) described, “... having family sort of understand when you need to be just focused in school... can be difficult to manage when you family expects you to have all these responsibilities."

Juanita seemed to express a sense of frustration as a result of her family not fully understanding the commitment required for her to excel at the University level. On the other hand, commuter students expressed the benefits of living at home and having a strong family support system, many of whom credit this continued and strong connection to family as the source of their motivation to achieve at school. Similar to the example Mary (1st Gen) provided about her family serving as a cheer team, Evelyn ( $\left.2^{\text {nd }} \mathrm{Gen}\right)$ specifically mentioned the re-energizing she feels from family gatherings when aunts, uncles, and cousins ask her about her experience at school and provide her with words of encouragement that increase her confidence to successfully navigate the institutional environment:

It [family gatherings] helps to take of the focus and then you see that you have all these people that are behind you, supporting you, love you...you remember like why you're working so hard towards achieving your goals. So you're able to go back in with a different - with your mind clear. Evelyn (2nd Gen) 


\section{Gender and Generational Factors}

While there was minimal mention of family support or challenges by the male students interviewed for this study (all of the males mentioned family support but only 4 provided the level of detail that was found in female responses), suggesting the male interviewees see themselves or hope to portray themselves as more independent and autonomous than the women who were interviewed. It seems to be a similar pattern as with the third gen students, which also makes sense, as they are more assimilated into the individualistic culture of mainstream America.

In addition, the comments about family support and challenges were much more prominent among the first and second generation, although they were discussed to some extent by the third generation students, many of whom emphasized how their parent's social and cultural capital served as a valuable tool for them as they navigated educational institutions. Overall, the more serious concerns that Raza students across generations discussed in regards to family support emphasize the need for institutional employees to gain a better understanding and develop action plans to address the limitations a diverse Raza student population encounters when asking for help, a result of a variety of lived experiences that shaped these student's perception of support. As students transition to the university, this notion of a "family away from home" rings true for a number of the students who took part in this study. 


\section{SENSE OF BELONGING}

\section{Support During Transition}

"Tenderly care about your well-being, your academics and anything between"

$$
\text { - Mary }\left(1^{s t} \text { Gen }\right)
$$

A number of students described components that would make up an ideal family environment at the institution. For Mary, there is a need for support systems that care about well-being, support, and anything between. The "anything in between" comment specifically touches on the need for an interdisciplinary or holistic approach to providing support for Raza students, with particular emphasis on the overall well-being of the student. For student like Emiliano ( $\left.2^{\text {nd }} \mathrm{Gen}\right)$, a sense of family is necessary to successfully navigate the institution, but specifically during the transitional period where students are adjusting to a new environment. Samuel ( $2^{\text {nd }}$ Gen) specifically discussed the transitional challenges that can be easily overlooked. He noted how sudden his transition to the university had been saying, "I wasn't even supposed to come here and all of the sudden, I'm here. It's hard to just be like, 'I'm going to do this..."' - Samuel ( $2^{\text {nd }}$ Gen) This narrative brings attention to the challenges associated with students who do not know they are going to college until weeks or sometimes a few days before they arrive at the institution, highlighting the need for institutions to explore how campus support can address the financial and other challenges that lead students to reconsider whether they are able to attend a university in the weeks and days leading up to the 
beginning of the semester. These difficulties were discussed specifically by first and second generation students who may not have had access to people with the cultural capital that could have helped these students address educational encountered as a result of not having access to the cultural capital necessary to adequately prepare for college, highlighting the need for this support across the Raza generational spectrum.

The discussion with interviewees about the need for support during transition also shed light on the lack of supportive relationships some of these students have had outside of the support they receive from family. For Sofia (2nd Gen), comments from friends about community college being better than a University lead to a sense of regret and questioning whether she belongs in the university environment. She spoke about a unique tension she encounters when discussing her university experience with her peer supports friends who are attending a local community college:

I can't even talk about school with them because it's just gets into a big argument at the end because they say, 'No, community college is better. I got all this [financial aid] money.' I kind of stay away from it just to avoid conflict. - Sofia ( $\left.2^{\text {nd }} \mathrm{Gen}\right)$.

This student highlights a very unique challenge where commuter students across generations deal with a dual environment that at times make it more difficult to navigate.

The dual environment consists of the institution and the home environment, two domains that may have very different components, values, and ways of thinking. If tensions between the home and institutional environment are not that if not compatible or addressed, students like Sofia may continue encountering feelings of doubt as they 
navigate the institution. Alondra (2nd Gen) also discussed unique challenges associated with a lack of support. She said, "first time I really received support from someone other than my family in my education space [when applying to college]".

This reality must be kept in mind as students across generation's transition and encounter a variety of support services but may seem hesitant to utilize them as a result of not knowing how the support can assist. One way to address this issue is by having support staff that understands the fact that students deal with transitional issues in different ways and at different paces. According to Juanita ( $\left.1^{\text {st }} \mathrm{Gen}\right)$, the role of support staff who serve as mentors is extremely significant for students like herself who come from an immigrant background with no family members who can help her navigate the university setting.

While support during the transition from high school or Community College to University is evident, students also discussed the importance of supports that help students prepare for the transition after graduating with an undergraduate degree. Julieta $\left(3^{\text {rd }} \mathrm{Gen}\right)$ specifically recommended support for graduating seniors that focused on transition to life after college, covering the emotional, career, financial, and life needs that students have as they move on to the next phase of adulthood.

One way that institutions can work to help students overcome transitional challenges is by encouraging students of similar backgrounds to share their narratives in an effort to help students normalize the struggles they deal with in college. While 
students like Juan (1st Gen) benefitted from a brother who shared his narrative normalizing the need to ask for help, a majority of interviewees did not reference a sibling with a college degree or having access to other people who could provide guidance based on their own narrative. Juanita $\left(1^{\text {st }} \mathrm{Gen}\right)$ did mention the positive impact of listening to the narratives of other students - she realized that she is not actually "crazy"; that the feelings she is dealing with are felt by others as a result of the countless stressors they are dealing with on a daily basis. According to Juanita, hearing these stories helped her develop a sense of comfort and led to an increase in her academic ability as well as her willingness to ask for support. She states that not having access to these narratives early on in her academic career at the institution led to an internalization of negative feelings that impacted her academic performance and well-being.

Julieta $\left(3^{\text {rd }}\right.$ Gen) also mentioned the importance of narratives to highlight the fact that students like herself are overcoming and are indeed graduating, stories that inspired her to stay strong through the process and reach out for support. Amanda ( $\left.1^{\text {st }} \mathrm{Gen}\right)$ specifically referenced the benefits of having a friend who continued her education into graduate school and how that served as motivation for her to continue on that path as well. She said, "That motivated me because of the person she is, and if she could do it, then I can do it. If she can come here and ask for help, then I can come here and ask for help." - Amanda ( $1^{\text {st }}$ Gen$)$ 


\section{Cohort as Family}

Beyond from the support needed during the transitional period to and from college, students described the significance of supports including cohorted classes and new college friendships as they continue their educational journey. Students like Carina $\left(1^{\text {st }}\right.$ gen) discussed the benefits of a peer support system and the multiple benefits it provides. One benefit is the establishment of friendships and the possible sense of an inclusive environment that is the result of positive peer relationships. In addition, for students like Carina ( $\left.1^{\text {st }} \mathrm{Gen}\right)$, peers serve as a classroom support. She described how she did not feel the confidence or entitlement to ask her professors for help, yet felt comfortable asking her peers.

Another student described his experience developing a positive relationship with community members who became like family:

They invite us over for dinner and stuff. They always want to make sure we're doing what we're supposed to be doing and I think that's pretty cool because we didn't even - all we were doing was moving for them. We never met them before. This was my freshman year, and they still call us and they ask us come over for dinner, just keep in touch - Jesus $\left(1^{\text {st }}\right.$ Gen $)$

While not all students will have the benefit of encountering a similar experience, the quote above emphasizes the need to help students establish some kind of family environment at the institution, possibly looking into the establishment of mentoring-type relationships between interested students and local families. A main component of this potential support would require people who, as described by 2nd Generation student, 
Valerie, "know where I'm coming from and they're willing to understand and be supportive..." Some of the interviewees have benefited from participating in a structured learning community with a cohort model where students register for the same sequence of classes within the same major. The students spoke about the multiple benefits of this kind of learning community, specifically the availability of tutors, the assistance in learning about and applying to internship opportunities, the impact on from a collective approach to solving problems, specifically in technical courses, and the overall structure support system the model provides. As Anna (2nd Gen) said, “...in my opinion there's really no need to go search out for other supports because that's already enough."

Students who were not part of a structured cohort that created a learning community expressed their desire for supports that could provide a structured environment for students to connect with each other to discuss academics, personal life, and anything in between:

It [conversation] could [be] about family, it could be about this one class that you're frustrated with that you're afraid of failing. It could be about like your whole experience I guess not just kind of this one identity about you like just being a student. - Juanita (1st Gen)

Other students spoke about the benefits of a structured buddy system, specifically allowing lower division students to connect with upper division students to get feedback about course difficulty and professor recommendations. This buddy system also serves as a tool for helping students feel more comfortable attending academic workshops and/or other support activities, as there is at times a sense of fear involved with attending a 
session/workshop where students will not know anyone: "It feels more comfortable to be with someone incase like you have to do something out of your comfort zone, you can have someone there to experience it with you." - Alondra ( $2^{\text {nd }}$ gen). A similar sense of fear was described by Juan (1st Gen) in relation to the fear of exposing himself as incompetent to support staff and Amanda (1st gen) who described the need to eliminate the fear students have about interactions with support staff. Gina $\left(2^{\text {nd }} \mathrm{Gen}\right)$ also described a similar limitation - her support seeking strategy is one that first has her exhaust all options (friends, classmates) before asking a professional staff member for help because she feels she has to sound really smart in front of the professors or other professional staff in supportive roles.

\section{Finding a Connection}

I feel like most of my support systems, I feel like I build a connection or I find similarities within the people in the support system - Evelyn $\left(2^{\text {nd }}\right.$ Gen)

In many cases, the mentorship, peer support, and family environment that were highlighted in earlier sections of this document have as one of the end results an increase in students' sense of belonging. In addition, the confianza that students require in order to open up about the various challenges students face often requires an authentic caring approach by those in supportive roles. The authentic caring can lead to the development of confianza which in turn can have an impact on students' sense of belonging. 
Interviewees have described the need for support services that have staff who are willing and able to develop a connection with students, who are personable, sincere, and take a proactive approach to addressing the needs of students. In addition to these qualities, students discussed the importance of developing a connection, not just in the sense of having the same major or interests, but more in the sense of a deeper connection where students feel like they have something in common with those in the supportive role. The connection is specifically significant for students who need to utilize academic and personal support resources but are intimidated or turned off by the lack of a connection. This need for a "connection" seems to be tied to a student's ability to see a part of themselves in the person who is providing support, in addition to breaking down barriers that result from negative interactions. The significance of positive interaction with support systems can help students become more optimistic about their education and the supports available to them. Just as students become pessimistic about support due to a negative experience, they also become more optimistic after positive interactions: "I usually come very positive out of every meeting that I have with my counselor and my peer mentor, and I take it throughout the whole day. I'm like, 'I'm really happy." - Sofia (2nd Gen)

Third generation students had much more positive comments in regards to their interactions with professor, as well as the support systems available at the institution. Martin $\left(3^{\text {rd }} \mathrm{Gen}\right)$ specifically emphasized how his professors welcomed questions and have been very supportive. On the contrary, Anna $\left(2^{\text {nd }}\right.$ Gen) feels like even when she 
does have conversations with professors, she doesn't feel like a majority of them are listening to her as she often gets this sense that they are not really interested or present in the moment when meeting with her. Ximena $\left(3^{\text {rd }} \mathrm{Gen}\right)$ also described her receptiveness to support services in her first two years of college and admits that support led her to develop more effective coping mechanisms to the point where she no longer feels she has to utilize the supports on campus as Senior - she stated that the support provided during her first two years helped her develop a sense of resiliency where she feels she is able to overcome challenges with little to no support, although she does admit that she knows there are services she doesn't take advantage of that would benefit her.

The need for positive interactions with faculty is particularly significant for students who are already coming into this educational setting with a pessimistic view of school support. Responses from students like Juan (1 st Gen) and Amanda (1st Gen), highlighted this sense of fear that is connected to professor interactions. Lupe ( $1^{\text {st }}$ Gen) specifically emphasized the need for professors to reach out to students who are not doing well in their courses. In addition to guiding students to appropriate resources, Lupe encourages professors to reach out to struggling students individually and make themselves available for support. Building on this, Mary $\left(1^{\text {st }} \mathrm{Gen}\right)$ mentions how she feels supported when support staff show interest in her well-being, her interests, and other aspects of her personal life. This issue of fear brought up by 1 st generation students may be tied to their lack of experience with the American educational institution in addition to past negative experiences with those in positions of power, an issue that was not 
discussed as extensively by third generation students specifically. The positive interactions that students have described often coincide with a sense of mentorship provided by the people in the support system. A number of students mentioned the role their mentors have played in helping them step outside of their comfort zone. Specifically, Gina ( $2^{\text {nd }}$ Gen $)$ mentioned the benefit of having someone support her who has faced similar challenges because they would have a better idea of how to help her overcome. In addition, Juanita ( $1^{\text {st }}$ Gen) believes people who can relate to her story are more likely to go above and beyond with their support as a result of knowing just how difficult the experience can be. She specifically referenced one faculty member who constantly went above and beyond to provide support to students like her. While she mentioned there have been a number of teachers across culture/gender that have supported her academically, not many faculty have taken the approach of support here as a whole student:

...so I think that that was the difference between this particular mentor that I'm talking about is that it's like a whole - like he doesn't only care about whether you pass his class or how strong your writing skills are... It's like the whole you kind of thing. - Juanita (1st Gen)

For Juanita, as was the case with Mary (1st Gen) it is necessary for those in support positions to take an interest in the whole student and, as the passage describes below, through a sincere approach: 
I consider myself a very intuitive person. I know that perhaps it may be the job of people on campus to make me feel comfortable and make me want to share information with them. But I guess what I'm saying is that it's not enough that they just tell me that I need to do that and that I could trust them. It's not just about that. For me I have to detect sincerity. Juanita (1st Gen)

For Juanita, being willing and able to provide support is not the determining factor in whether or not she utilizes support. For her, and many others, there is a need for a sense of sincerity, meaning students like Juanita are looking for support that is led by people who are both honest and sincere. Building on this, Valerie $\left(2^{\text {nd }} \mathrm{Gen}\right)$ mentioned the need for support staff that approach her in a way that keeps in mind the challenges Raza students like herself face in navigating an institution that at times is incompatible with their home culture:

You're not going to feel comfortable telling them, 'Yes, I'm having academic problems.' You're not going to be comfortable coming to somebody like that. - Valerie (2nd Gen)

Julieta $\left(3^{\text {rd }} \mathrm{Gen}\right)$ also emphasized the need to have support staff that are easy going and don't have to pretend like they have everything figured out. The welcoming vibe she got from the professor made her more approachable. This is contrary to other examples provided about intimidating professors who discouraged her, although she acknowledges the professors themselves may not be trying to intentionally intimidate their students. Evelyn (2nd Gen) also described the need for people in supportive environments who have the right attitude. She emphasized the attitude is a determining factor on whether or 
not someone is actually qualified to serve in a supportive role. Samuel $\left(2^{\text {nd }} \mathrm{Gen}\right)$ also discussed the importance of getting a good vibe from those in supportive roles because the feelings they encounter as a result of the interaction with support staff will dictate whether the students utilizes the support in the future. This vibe seems to be connected to an authentic sense of caring. Juanita $\left(1^{\text {st }} \mathrm{Gen}\right)$ posed the question she has in mind every time she interacts with those in supportive roles - "...does this person really care?" Further, Sofia ( $2^{\text {nd }}$ Gen) mentioned that a support system could have valuable programming and resources, but if the person behind the program does not seem caring, then she is less likely to utilize the resource. This reality has large implications for both students who would benefit for support services as well as programs whose objective it is to address various student needs. Additional students like Jesus ( $\left.1^{\text {st }} \mathrm{Gen}\right)$ spoke about the need to have a firm support system - just because students are looking for a caring and warm support system, doesn't mean that they don't want someone who is also firm:

"Truth is a brutal but sometimes you just really need to hear it." - Jesus ( $1^{\text {st }}$ Gen $)$

\section{Marketing/Branding of Services}

“...we don't know all the questions... sometimes we are misinformed..."

$$
\text { - Mary (1 } 1^{s t} \text { Gen) }
$$

As described in the previous sub-section, the way support services are packaged has an impact on whether or not students will take advantage of the resource. Another issue with regards to support is the way the services are marketed to students. In some 
cases, students (both on-campus and commuters) may feel like the support isn't relevant to them because of the way it is marketed or perceived.

Amanda $\left(1^{\text {st }} \mathrm{Gen}\right)$ specifically discussed being told that certain research services on campus were not open to all students. In addition, Ximena $\left(3^{\text {rd }} \mathrm{Gen}\right)$ in particular seemed to believe that utilizing supports on campus would somehow take away from time that they could be spending on their academics. Ximena seems to make a distinction between support and academics, separating them into two separate categories. There is this sense with Ximena that student's feel they have too much on their plate to utilize support because that would take time away from homework. At the time of the interview, this student does not see how support services, specifically those personal/emotional supports, can have a positive impact on her academically. Emiliano (2nd Gen) discussed a similar point by emphasizing that he only takes advantage of academic support because it's a necessity, as compared to emotional support which he described as "I don't feel it's a necessity...that it's something crucial in my life that I need." Emiliano did acknowledge later in the interview that there's a lot that goes on in his life and that he knows he would benefit from speaking to a professional but at this time, he doesn't because he doesn't see it as absolutely necessary. The three examples provided above go across generations and seem to highlight this disconnect Raza students have about the support available as well as the connection between emotional support and academic performance.

In addition, Daniela (2nd Gen) discussed how this mentality of "support not being for me" may impact non-Raza students: 
I've have a lot of Hispanic friends that are doing so well because they go all the support services whereas a lot of non-Hispanic friends I've never really heard of them go to any of these support services. I feel like they've [Raza] kind of got it figured out. - Daniela (2nd Gen)

Daniela sees that some of her non-Raza friends are encountering greater challenges than her Raza friends as a result of not utilizing support and this also has an impact on her perception of support. Similarly, Gina ( $\left.2^{\text {nd }} \mathrm{Gen}\right)$ recommends moving away from a model where there are support programs that target specific populations. Instead, she recommends supports that are open to all students and don't have a label because according to her, the label is what stigmatizes the services and thus makes it harder for students to utilize.

Lucas $\left(2^{\text {nd }} \mathrm{Gen}\right)$ also spoke about the factors that discouraged him from seeking out specific support services, specifically applying for scholarships. Since he is not fully informed about the process, he has this belief that it is not likely he will receive a scholarship because so many people are applying and this belief is what serves as a barrier for him. He could have a fear of rejection on one end or there can also be a sense of not feeling good enough to apply. This fear of rejection is slightly different than the sense of fear students described earlier as it relates to communicating with support staff. For both examples, there is this sense of a lowered self-worth tied to what they perceive will be a negative outcome for moving forward with these decisions - Lucas has the fear of not receiving the scholarship while students like Juan (1st Gen) and Amanda (1st Gen) have a fear of how they will be perceived. Both examples are directly tied to self-worth. 
Julieta ( $3^{\text {rd }} \mathrm{Gen}$ ) also brought up the challenges associated with not knowing what to say when communicating with people in support positions.

Daniela ( $2^{\text {nd }} \mathrm{Gen}$ ) brought up the issue of being misinformed about some of the supports on campus, specifically seeing the supports as necessary for only those students who are seen as a deficient. This way of thinking can create barriers between students and supports since most people do not want to be labeled as deficient. Isabella ( $\left.2^{\text {nd }} \mathrm{Gen}\right)$ also reiterated this belief that certain supports were only for students who are English as a Second Language (ESL). She also mentioned this notion of "not feeling included." While Isabella did not elaborate on what she meant by that, the issue of not feeling like support services are inclusive of her needs is directly tied to the third research question for this study that aims to identify what supports are desired by Raza students.

Ximena $\left(3^{\text {rd }}\right.$ Gen) specifically cited her misunderstanding of certain support services only serving students who a remedial in English and/or math. Daniela ( $2^{\text {nd }} \mathrm{Gen}$ ) brought up the issue with learning about services only via email. She went on to say that most students don't read the entire message in such an email, and so the institution could have a greater impact if it marketed such services in different ways, for example through classroom presentations or tabling. 


\section{INTERNALIZED OPPRESSION}

\section{Challenges in Reaching Out}

"I didn't reach out. I waited until I was reached out to. When I was forced to come in...not wanting to put myself out there as somebody who can't help herself..." - Valerie (2nd Gen)

The statement above speaks volumes about the limitations and challenges some students encounter in an educational system that is set up to be reactive, to wait for students to make the first move in regards to seeking support. Valerie's statement is just one of many highlighting the various factors involved with taking advantage of resources. The issue of fear has been discussed by a number of students who emphasized the reservations they encounter when deciding on whether or not they take advantage of certain resources or support systems. The findings of this study suggest that the fear students have described is part of a larger challenge students face as they deal with the shame and internalized oppressor that is the result of a variety of lived experiences

Valerie's quote about not wanting to be seen as someone who can't help herself brings up an issue that is two-fold: there was shame involved with seeking support and also a desire for support staff to show interest in her before she opened up about the challenges she was dealing with. This interest was referenced by Valerie, who explained she wished faculty would encourage them to utilize appropriate resources, since faculty may have a good sense of students' needs based on their performance in the course. She states that while she understands that students are expected to take advantage of resources 
on their own without waiting for someone else to help them, faculty need to realize that this approach is not realistic for students who have reservations about support systems and/or those who have dealt with life and educational experiences that have a direct connection to their approach to getting help. Martin $\left(3^{\text {rd }} \mathrm{Gen}\right)$ also spoke about the significance of a pro-active approach by those in supportive roles. He mentioned there is quite a difference in whether a support staff is letting students know they are available to support, as in, "Let me know if I can be of support to you," versus supports that take initiative and ask "How can I help you?" instead of "Let me know if I can be of support to you." The two statements can be interpreted in different ways, specifically for students who already have reservations about seeking help.

In regards to gender, Valerie $\left(2^{\text {nd }}\right.$ Gen $)$ described the notion that it is easier for females to ask for help as a result of living in a society that glorifies the "Disney Princess" that according to her, is dependent on others to fulfill her needs - she admits that this reasoning for asking for help is flawed because there may be times when females ask for help without first trying to solve the problem themselves. Similarly, Victoria $\left(3^{\text {rd }}\right.$ Gen) mentioned what she described as traditional gender roles where females ask for help while males are more accustomed to providing support, hence why males may have a harder time asking for help. Valerie $\left(2^{\text {nd }} \mathrm{Gen}\right)$ reiterated the male support-seeking challenges as a result of them following a "go getter" mentality, where males do things on their own and do not ask for help. Another challenge in reaching out was described by Felipe ( $2^{\text {nd }}$ Gen), who mentioned wanting faculty and support staff to pick up on "hints" 
the student is providing as a call for help. He said, "If I just mention a couple of comments and you kind of pick up on like, 'He needs help that's why he's trying to say.' that's when I feel supported." Felipe suggested this is a practice some students engage in as a way to not put themselves out there as needing support. Students are thus expecting support staff to always be in the "I'm ready to help" mindset.

Julieta $\left(3^{\text {rd }} \mathrm{Gen}\right)$ also spoke about her approach to seeking support and wanting staff/faculty to figure out the help she needs because for the most part, she doesn't know what she needs and thus expects those in supportive positions to be able to figure out her needs based on pieces of information she provides. This approach of having faculty and staff guess what Julieta needs is tied to her own confusion about what support or resources are out there to assist her. In regards to Raza generational challenges specifically, Amanda described the lower expectations that students like herself face as a result of the $1^{\text {st }}$ generation label. For her, the reaching out to this population is very important because in many cases, these students do not know where to start when wanting to seek assistance. Daniela, a second generation Raza student, had different views on this issue. According to Daniela, the $1^{\text {st }}$ generation students are more likely and willing to ask for help since they seem to have this belief that they will not be successful unless they ask for help, “...first generation students are looking for help across the board because they know they probably know they need it." On the contrary, she feels that $2^{\text {nd }}$ generation students like herself face more challenges in asking for helping for help, one of those challenges being less willingness to reach out for the supports that are available. 
Isabella $\left(2^{\text {nd }} \mathrm{Gen}\right)$ also discussed additional challenges in reaching out as a result of what she perceives to be support services that are tailored more towards $1^{\text {st }}$ generation students, a claim that is similar to her comments earlier about thinking that certain services were only for ESL students and Ximena's (3rd Gen) comments about support services only serving students who are remedial in English or Math. The issues of second and third generation students perceiving supports as only serving ESL and remedial students is tied to the first research question of this study focused on identifying supports that Raza students perceive as valuable.

In contrast, $3^{\text {rd }}$ generation students overwhelmingly shared narratives highlighting the various supports they had from both parents and teachers. Martina $\left(3^{\text {rd }} \mathrm{Gen}\right)$ spoke about a strong parent support system from a very young age, as he saw his parents actively engaged in his education. Victoria $\left(3^{\text {rd }} \mathrm{Gen}\right)$ also spoke about the benefits of having a mother who worked at the community college and thus was able to provide valuable support to her daughter as she was getting ready to apply to colleges and complete her financial aid application.

\section{Fearing the Implications of "Discrete" Services}

"I feel like if it just like more friendly if people didn't make it seem like it wasn't such a bad thing to go ahead and look for a service and it would be easier to do so."

- Daniela $\left(2^{\text {nd }}\right.$ Gen) 
While students on campus have been encouraged to utilize the personal supports on campus, some of the Raza students interviewed expressed barriers in utilizing supports that they labeled as "discreet." For some Raza students who grew up believing there was a stigma tied to seeking emotional/mental health support, there is concern when the university markets some services as discrete. Four of the students interviewed specifically noted that this label set an additional barrier, even though they knew they could benefit from the emotional/mental health services offered on campus. Additionally, two students discussed the fear of their utilization of mental health services going on their record, something they believe will make others believe they are weak. This fear associated with not wanting others to find out about their utilization of certain services can also be tied to how the emotional/mental health services are advertised.

For example, Daniela $\left(2^{\text {nd }} \mathrm{Gen}\right)$ mentioned that while services are available for those students who are dealing with thought of depression, they are labeled as "discreet" and that is a problem because it makes it seem like those who are dealing with actually have a problem. The "discreet" label also makes it seem like a particular student is the only one dealing with the issue, which is far from the truth. Daniela would prefer if the issue of depression was advertised differently, in a way where students can feel comfortable about talking about their issues since they know others are dealing with similar issues. Further, for Raza students who rely on a strong sense of family, there are benefits to packaging mental and emotional health services in a more collective and familial framework. According to Daniela, moving away from the "discrete" approach 
can have a positive impact on students, particularly those who are dealing with suicidal thoughts. In her opinion, it is necessary to talk about these issues before tragedy strikes:

They don't start speaking about the support services they have until something tragic happens. I think that's mainly what really bothers me. A lot of things like this could have been prevented. I wish being depressed or being upset like was not put in such a negative manner, depression. I know a lot of people with depression, it's okay to be depressed. It's not something that you want. That's probably not something you want to share with everyone. That's not something that you should keep this to yourself. - Daniela (2nd Gen)

Daniela specifically mentions that depression is not something she would want to share with others as a result of the way the services are packaged as discrete. Daniela also mentioned that there are ways for depression-related support services to first be provided by programs who do not necessarily specialize in that field, allowing the student to discuss initial concerns with someone who doesn't have the label of a therapist or medical practitioner. Once there is a sense of comfort about speaking on the issue, the student can be referred to more specific and/or intensive services, but the initial discussion must take place in a space where the student does not feel intimidated. Felipe (2nd Gen) provided an example of a program that according to him, helps students discuss challenges without shaming them. Felipe's statement contradicts Daniela's statement but it must be noted that Felipe is referring to those support programs that encompass academic/personal/career support, while Daniela was specifically referencing the support on campus that focuses on mental and emotional health. "...I I've seen these 
programs as a good thing because they're ready help and not to shame me or anything in anyway."

\section{Impact of Pre-College Experiences}

"I know when I was having trouble, my sister was like, 'Why don't you try go into, like see the school psychiatrist?' I was, 'No, if I do it and I know my reasons, they going to think I'm crazy. "' - Daniela (2nd Gen)

Unfortunately, many of the students who were interviewed described challenges in seeking support as a result of various limitations, some of which are connected to past life experiences while others are connected to the negative interactions they've had with people in supportive roles throughout their educational career. Similar to the challenges associated with utilizing services that are marketed as discreet, some students hesitate to utilize support for fear that they may be seen as weak, deficient, or as Daniela put it, crazy. Daniela (2nd Gen) describes not really knowing why she has this belief that people will think she is crazy if she sees a therapist, but says that even though she knows the services will be helpful, she finds ways to discourage herself from going.

In addition to wanting to avoid this sense of being weak, students also express their fear of being judged as a reason for not seeking support. Juanita ( $1^{\text {st }}$ Gen $)$ mentioned the need to avoid support services because they make her vulnerable and get her thinking about all of the things that are supposedly wrong with her. She also then victimizes 
herself as the only one dealing with the problem, a move that further complicates her ability and willingness to ask for help. She acknowledges that even though she realizes the support could benefit her, the fear of embarrassment, being labeled, and not knowing others who have utilized similar services ultimately discourages her from taking advantage of the service.

Anna ( $2^{\text {nd }}$ Gen) described "not wanting to feel as a burden" as the main reason for not seeking support. Similarly, Alondra $\left(2^{\text {nd }}\right.$ Gen $)$ discourages herself from utilizing that specific support as a result of not wanting to attend solo as a result of fear of an unknown environment in addition to the fear of finding that something is "wrong" with her. Other students spoke similarly about this fear associated with being labeled as someone who needs additional support. Building on Daniela's $\left(2^{\text {nd }}\right.$ Gen) comments earlier about wanting services to be seen as less discrete, Felipe ( $\left.2^{\text {nd }} \mathrm{Gen}\right)$ expressed concern about someone finding out that he has utilized certain mental health services. These fears may also be tied to him not wanting an easy way out.

I don't want them [support staff] to make it easier for me. I wanted to go in there and attack this thing and make sure that I did it without getting the easy path, like make it sure I took the path that I was supposed to take and I wasn't -- I could give that special treatment because of what was going on in his life. - Felipe (2nd Gen)

Felipe not wanting an easy way out may also be tied to the challenges faced by his parents, particularly his mother, while growing up. Since his mother faced various setbacks and challenges and was still able to overcome without much support, he feels he has to follow the same path of dealing with the challenges on his own. This feeling of 
"not wanting to let my parents" down is also evident in other students who referenced their parents in the reasons that keep them motivated:

...my mom when I came to the university she told me, 'If you want to go back [home] someday, It's no problem...I took that as a challenge like, You know 'I can do this,' like, 'I'm not going to give up.' - Mary (1st Gen)

This fear of being seeing as weak as described by Mary can be tied to the struggles of the students' parents as well as the expectation parents have for their children in college - students may hesitate to ask for help as a result of thinking they are letting their parents down if they do. In addition, as is the case with Mary, she feels she has something to prove to her parents by being successful in college and not showing any weakness or fear. Amanda ( $\left(1^{\text {st }} \mathrm{Gen}\right)$ also spoke about the impact her mom's own struggles have on her ability to ask for help. She refers to her mother as a strong and independent woman who was able to accomplish many things in her life without much support. When asked about why she doesn't ask for help, Amanda acknowledges that in addition to not feeling smart when she asks for help, she knows that the sense of independence she got from her mother serves as a barrier for her to seek help.

I guess it's really hard for me to ask for help. I don't know why. I don't understand it, because I would rather struggle and write a paper by myself when I could go in and get help from somebody. I don't know why. Amanda (1st Gen) 
Other students also highlighted the internal barriers they face when asking for help, some of which are tied to life experiences that took place before the student arrived at the institution. Ximena $\left(3^{\text {rd }} \mathrm{Gen}\right)$ finds herself having a pessimistic view of support as a result of past negative experiences. She felt that people are only willing to help because it's part of their job and not because it's something they actually want to do. This belief had a negative impact on her for many years as she convinced herself that those in positions of support did not want to be there.

Daniela ( $2^{\text {nd }}$ Gen) similarly expressed her challenges in asking for help as a result of negative experiences in her childhood; she remembered being laughed at by her peers when she asked for help. She mentions that this and other negative experiences led her to develop a mentality of not wanting to ask for help for anything. This approach has led to frustrations when she does need assistance, because negative experiences from the past continue to serve as barriers. While Anna $\left(2^{\text {nd }}\right.$ Gen) expressed that she is now utilizing various services on campus, including personal health services, it took her close to 4 years to get enough confidence to utilize the service. She mentioned that she had positive experiences with other supports on campus and believes that it was these positive experiences that ultimately led her to reach out to additional services.

In contrast, Amanda ( $\left({ }^{\text {st }} \mathrm{Gen}\right)$ specifically spoke about a negative experience with a tutor on campus:

He [tutor] made me feel really bad because he was -- I mean, he was just really smart, and he would brag about it, and I didn't like that. Not 
bragging -- I don't know. I mean, just being humble, like, 'Yes, you're smart, but don't like rub it in my face,' kind of thing. - Amanda (1 st Gen)

Amanda highlights how these comments may be internalized by students, leading to a shame that makes it more difficult to ask for help in the future. In addition, negative interactions can then have a domino effect where students make assumptions that no support staff actually care about them as a result of one bad experience. Lupe $\left(1^{\text {st }} \mathrm{Gen}\right)$ highlighted this sense of "They don't care about the student. They just care about getting paid". While Lupe did not mention why they have that belief, it is likely this is a result of past negative interactions with people in supportive roles. The need for support staff to care is tied to the need for sincerity that Juanita (1st Gen) and Valerie (2nd Gen) discussed in earlier sections of this chapter. Juanita and Valerie's example is important to keep in mind as institutions work to ensure that students do not get to the point where Lupe (1st Gen) is, where are pessimistic about the true intentions of those in support environments. It can be said that Lupe developed this way of thinking as a result of not having positive experiences with support staff in the past.

Other students like Alondra $\left(2^{\text {nd }} \mathrm{Gen}\right)$ discuss the challenges associated with seeking support from people from different ethnic backgrounds. She mentioned a bad experience with a Caucasian English teacher in elementary school and how that specific experience traumatized her for a significant amount of time. When she encountered a Caucasian in a supportive setting in college, she says she could still feel the sense of fear and discomfort that was rooted in that past experience. She says while the Caucasian who 
helped her in college was indeed helpful, she has hesitated to go back. While students like Alondra ( $\left.2^{\text {nd }} \mathrm{Gen}\right)$ and Amanda ( $\left.1^{\text {st }} \mathrm{Gen}\right)$ acknowledge that they are open-minded about receiving support from people of diverse ethnicities and cultures, they both mention feeling self-conscious about going into a place that is majority Caucasian:

When I'm like surrounded by Caucasians, it's a little intimidating. I don't know why. I haven't been discriminated against, really. It's just something in the back of my head. I don't know. I really can't explain what it is. It's also me. It's just hard to get in there, but it's even harder if -- I don't know. It's just -- it's something in the back of my mind, and then it feels uncomfortable. I don't know what it is. - Amanda (1st Gen)

Amanda's inability to point out the exact reason why a Caucasian dominated environment makes her uncomfortable raises questions about how she has subconsciously internalized past experiences where microaggressions could have been encountered. As was the case with Alondra's discomfort in seeking support from a Caucasian support person, Amanda also described a past experience where she felt her Caucasian male tutor made her feel dumb as a result of what she interpreted as him bragging about his academic ability, an issue that Samuel (2nd Gen) and Juanita (1st Gen) pointed out when discussing the need for support staff that are guided by an authentic sense of caring.

Many of the interviewee responses in regards to limitations in asking for help also highlight a sense of independence that students pride themselves in. The sense of independence can be seen as a strength but it can also be seen as barrier for students who have too much pride to ask for help. The sense of independence has a variety of root 
causes. Amanda ( $1^{\text {st }}$ Gen $)$ discussed that she never witnessed her mother asking for help and aspires to be a strong and independent women like her. As a young student, she developed this belief that asking for help meant she was not a good student, another mental barrier that she has had to overcome. For Juan $\left(1^{\text {st }} \mathrm{Gen}\right)$, even though he has asked for help on various occasions during college, he developed this belief that asking for help meant that he was too lazy or too dumb to figure things out on his own, and this in turn made him develop a stronger sense of independence. Jesus ( $\left.1^{\text {st }} \mathrm{Gen}\right)$ also states he likes to do things on his own, referencing the academic achievements of his parents even though they faced language barriers. He feels he is not living up to his parent's expectation and work ethic if he is asking for help. He acknowledges utilizing the tutoring services on campus and even though he received help, he states "it just didn't feel right." Other students like Camila ( $\left.2^{\text {nd }} \mathrm{Gen}\right)$ reference growing up independent and learning to rely on themselves for various needs from a very young age. While Camila developed this sense of independence as a result of positive support system from family, Samuel ( $\left.2^{\text {nd }} \mathrm{Gen}\right)$ developed a forced sense of independence as a result of growing up in what he refers to as an unsupportive environment:

I always grew up in an unsupported environment so I always learned to do things on my own. I learned how to cook on my own. I learned how to play soccer on my own. It was just an independent -- I've been independent since I was like five honestly. I've never really wanted ask for help...Yeah, I'm still like that. I'm still stubborn. I still want to do it my way. - Samuel (1st Gen) 
Joaquin $\left(2^{\text {nd }} \mathrm{Gen}\right)$ also discussed the tension with being independent and seeking support and said he addresses that by acknowledging that he still needs to put effort on his part when asking for help, which seems to somehow reassure him that he is not asking for help as a result of laziness or lack of work ethic.

Interviewees described additional ways they are able to justify not seeking support for various academic and personal needs. Camila ( $\left.2^{\text {nd }} \mathrm{Gen}\right)$ mentioned she only seeks support when dealing with a "major problem," an approach that raises concerns for institutions who would like for students to transform their view of support into one where you ask for help when things are going well, not only when things are going really badly. Other students like Carina ( $\left({ }^{\text {st }} \mathrm{Gen}\right)$ discussed the pressure of her parents as a reason for not seeking support:

...my mom always tells us that if we're not the best then you're not doing anything good in a way. It's always been you're doing everything really good or you're not doing well at all. - Carina (2nd Gen)

For Carina, seeking support is counter to the "first place" mentality that she learned from her parents. This "first place" mentality was also developed by other students who mentioned that they have trouble seeking support because they are not used to asking for help - they mention being in AP classes in high school and doing well. To all of a sudden be in a position where they need assistance, it's a change for them and something that takes some time to get comfortable with. As Carina (1st Gen) said, "When I came here and I found myself needing help, it was really hard for me to reach out for it. Yeah, it's definitely hard." 


\section{Hood Mentality}

They [male friend] kind of just told me people don't care about, like people from where we're from. They told me you're going to do it on your own because no one's going to help you. Now that they do offer help in school, I think I just turn them down [offers for help] a little bit [as a result]. - Jesus (1st Gen)

A few of the men specifically expressed certain challenges associated with seeking unique kinds of support. Jesus $\left(1^{\text {st }} \mathrm{Gen}\right)$ specifically referenced his circle of older friends, many of whom served as mentors for him, even though many of them engaged in unhealthy lifestyles and criminal activity. Those older friends planted in him a view of the world that has made it more difficult for him to trust others.

Another male interviewee Emiliano also acknowledges limitations as a result of growing up in an environment that expected males to be strong and not need support from a "shrink." This student acknowledges that he would benefit from seeing a counselor as a result of the many traumatic experiences he has had in his life, but he admits he hasn't gotten to that point where he is willing to take that next step.

...we don't see ourselves from where we grew up talking to what we call a 'shrink.' But in reality, that would benefit me. There's a lot of stuff that goes on inside of my mind where families and issue outside or issues where everything builds up...- Emiliano $\left(2^{\text {nd }} \mathrm{Gen}\right)$

Emiliano went on to discuss this "know it all" mentality that was prevalent in the "hood" he grew up in. To make matters more challenging, he also experienced what he 
believe was discrimination and a lack of support as a result of being a Raza student in a Caucasian dominated high school, a reality that also led him to have additional reservations about asking for help. For Emiliano, it wasn't until he was the victim of gun violence that he began to change his way of thinking in regards to his education and the importance of support as he pursues that educational journey.

Asking for help, it would have been ideal but living in the neighborhood, we never did because we -- I guess we had a view that we don't ask for help. We do it ourselves. Coming from a bad neighborhood, we think we know it all. That's where it's wrong, we don't. - Emiliano (2nd Gen)

\section{Chapter Summary}

The three themes of "family away from home," "sense of belonging," and "internalized oppression," capture the experiences and needs of the institution's Raza students and address the study's research questions about institutional support. The themes focused on family and sense of belonging emphasize the supports that are valuable and currently effective for students at this HSI. Students' comments also highlighted some challenges associated to those themes, particularly challenges encountered as a result of negative experiences within the home (lack of support from family, limited access to social and cultural capital, minimal experience with support) and institutional domains (fear of utilizing services, deficient view of support). The theme focused on internalized oppression highlights the additional weight students carry by the 
time they arrive at this institution, how the shame/internalized oppression they feel is at times reinforced at the institution, and the need for universities that serve Raza students to focus on sense of belonging and building a family-like environment to address internalized oppression.

In addition, the six components that are the focus of the cultural wealth model were all discussed by the interviewees in various ways - for example the familial references (by Juanita, Carina, and Juan, among others) and the navigational references (by students like Anna, Evelyn, and Felipe) - reinforcing the potential for the cultural wealth components to be adopted into institutional practices and policies that are aimed at improving student success. For example, the cultural wealth focus on developing students' aspirations was highlighted by student comments about the need for staff who go above and beyond through an authentic sense of caring to mentor and guide students. In addition, the confianza and sincerity that was discussed as necessary would also lead to positive outcomes for student aspirations as they will have a stronger sense of support. The potential for narrative sharing is also tied to aspirations as students can have higher aspirations after hearing the narratives of other students from similar backgrounds who have had similar experiences and were successful.

For the linguistic component of the cultural wealth model, there is an emphasis on understanding the capital students bring with them, a point that came to light throughout the three themes - the need for support staff to understand the support-seeking limitations of students; the need for support systems that match the family support environment 
students have at home; mentors who have an understanding of the cultural background of the student; and the sharing of narratives to help students normalize the struggles they encounter. The familial component of the cultural wealth model focuses on the significance of a sense of family for students, a point that was also present throughout the two themes of "family away from home" and "sense of belonging."

The social capital component of the model focused on the need for peer support systems, for all students but specifically for commuters who are constantly navigating between two environments that are in most cases vastly different. The commuter students in this study emphasize that point of having a strong support system, of connecting with other commuter students to have a space where they can discuss challenges that are specific to their group as well as opportunities for developing their school family network. Similarly, the navigational component of the model emphasizes the need for institutions to examine what practices and policies are both helping and hindering student progress. Specifically, the navigational component requires the institution to acknowledge that policies and practices that are detrimental to students. This issue was discussed across the three themes, by commuter students who feel a support system is lacking for that group, by students who feel that services are marketed in a way that makes certain students be more intimidated by the support, and by students who are unable to explain the reasons they are not utilizing support, a phenomena that fell under the "overcome internalized oppression" theme. Finally, the resistance component of the cultural wealth model is highlighted in student responses about the value of narrative 
sharing, where students and staff pass down knowledge to students of similar backgrounds. Even if the students in this study did not directly mention how the positive or negative support experiences will impact how they share capital with other students in their home communities, the long-term societal benefits of Raza students having positive experiences with supports deserves additional attention as the Raza population continues to grow and more Raza enter higher education - the need for incoming students to hear the narratives of those that came before them and the availability of mentors can have a drastic impact on future graduation rates for this population.

In addition to the overlap between the cultural wealth model and study themes, there were also similarities within the three themes. The first theme of family support went across all three of the themes as students described both the benefits and challenges of the family support system. For students like Felipe (2nd), the strength and motivation he gained from his parents struggled also led to a fear of seeking support as he felt he needed to struggle through the challenges just as his parents did. Similarly, students like Jesus (1st Gen) and Camila (2nd Gen) mentioned the sense of independence they gained from family as a strength but it was also this sense of independence that created a mental barrier for them in times when they should have been asking for support. For students like Gina (2nd Gen), Anna (2nd Gen), Samuel (2nd Gen), and Ximena (2rd Gen) who mentioned challenges as a result of growing up in an environment that wasn't supportive, these students mentioned additional difficulties in utilizing the support services on campus, although their reasons varied. It's important to point that that 3rd Gen students 
like Ximena continue to encounter difficulty in transitioning to college, a reality that counters many beliefs about 3rd generation Raza students having access to capital that more adequately prepares them for college. That being said, family support challenges were discussed more often by the first and second generation students who participated in this study.

For males specifically, they were less likely to bring up the family support system, although first generation males like Juan and Jesus did make more mention of family support. For the others, there is the possibility that they did not discuss family support as much due to their sense of independence and wanting to figure things out on their own. In addition, males Jesus (1st Gen) and Emiliano (2nd Gen) specifically discussed the challenges in reaching out as a result of the pressure of maintaining traditional male gender roles that required them to not be portrayed as weak.

In regards to the authentic caring and sense of belonging that students referenced as necessary to help them feel more comfortable utilizing supports, students across generations and genders spoke openly about the need for support from mentors, peer supports, and the sharing of narratives by both peers as well as support staff that serve as mentors. Juanita (1st Gen) specifically mentioned the significance of mentors and peer supports for first generation students who are transitioning to the University setting while Julieta (3rd Gen) discussed the need for mentors to assist students to prepare for life after college. Although both discussed the need for mentors for different reasons, the significance of mentors and peer supports is significant for students across generational 
status. In addition, mentors can potentially address the needs of students like Victoria (3rd Gen), Felipe (2nd Gen), and Valerie (2nd) gen who expect support staff to be more pro-active about providing support for students - if a strong mentoring relationship is developed, support staff are more likely to get a better sense of the needs of their mentees. For males specifically, Barajas and Pierce (2001) have found that mentoring relationships are more beneficial for males than the support they receive through peer-topeer interactions.

The issue of students feeling a sense of belonging is extremely significant as a result of the implications of a negative experience for students. One negative experience can lead students to shut down and thus it is important to acknowledge the significance of positive experiences as soon as the student arrives at the institution. The result of the student experiences determines the role the "internalized oppression" theme will have on students as they continue their education. The fear of failure and shame that is associated with this theme was discussed prominently by first and second generation students, with third generation students having more positive examples of support that then limited the amount of students in that group who discussed support seeking limitations. Staff must also understand that student reservations about utilizing support, which was discussed by Anna (2nd Gen) and Daniela (2nd Gen) among others, can be tied to past life and educational experiences. For students like Gina (2nd Gen), her mention of wanting support services to be open for everyone can be tied to the point Daniela (2nd Gen) discussed about the discrete label for services serving as an additional barrier. It seems 
like the exclusivity of services, which some may see as a strength, can also serve as a barrier for students who don't want to feel targeted by support services.

Ultimately, the study themes of "Family Away From Home," "Sense of Belonging," and "Internalized Oppression" all encompass challenges as well as opportunities the institution can address intentionally through a variety of approaches and strategies that will be discussed in the following chapter. In addition, as the institution works to address issues of belonging and internalized oppression amongst the Raza student population, it will be important to keep in mind the unique needs of Raza students across gender and generational status. 
Chapter Five: Discussion and Conclusions

\section{Overview}

While HSIs were not established to specifically serve our nation's Raza population, they are now seen as playing a key role in our country's ability to increase college graduation rates, specifically for underrepresented populations (Lee Jr. \& Keys, 2013). As a result, there is now more emphasis on examining HSIs to ensure they are living up to the Raza "Serving" label. There are currently extensive disparities in knowledge about HSIs, challenges the institutions face, and the impact they are having on student performance (Hurtado \& Ruiz, 2012). According to Malcom-Piqueux \& Bensimon (2015), institutions must become more committed, if the HSI label is to be credible and have significance for students, educators, and those involved in policy. As the Raza population in the United States continues to rise, HSIs are positioned to positively impact the degree attainment rates of Raza students in the U.S. (Galdeano et al., 2012). In turn, the education and qualifications that coincide with a college degree can lead to Raza advancement at all levels of government and society. Unfortunately, research has found that many Raza students at HSIs experience an educational environment that leads to inequitable academic outcomes and has a negative impact on their persistence and graduation rates (Malcom-Piqueux \& Bensimon, 2015). In the spirit of an equitable approach to improving educational outcomes for Raza students, there is a need to focus on institutional practices and their impact on Raza students, a shift from approaches that unintentionally blame students for shortcomings in educational 
performance instead of the practices and policies of an institution (Malcom-Piqueux \& Bensimon, 2015). While improvements in educational outcomes may be a challenge as HSIs are underfunded and serve a large percentage of students with various needs (Galdeano et al., 2012), it is necessary to examine what can be done, given the current context, to increase degree attainment rates and student development for Raza at HSIs.

In an effort to examine components that impact student success at an HSI, this study focused on exploring perceptions of support at an HSI in Central California, with a specific focus on examining the supports that contribute to a positive University experience as well as recommendations for additional support needs for a multigenerational Raza student population. The study incorporated a qualitative research design, although the first phase of the study utilized quantitative methods to capture demographic information for Raza students at the institution. Of particular interest was information on student generational status and gender to gain a better understanding of the impact, if any, of generational status and gender on students' perception of support. Of the 2,303 identified Raza students who received the survey, 370 students responded, representing $16 \%$ of the Raza student population at the institution. Once a representative sample (based on generational status and gender) of Raza survey respondents was identified, 25 were interviewed for the qualitative component of the study. Interviewees were asked a series of 10-15 questions covering aspects of the student experience with support prior to entering the institution, their support experiences on campus, and desired support systems and services. 
The testimonies of these 25 students covered a wide range of experiences involving support throughout their lives, all influenced by family and cultural dynamics, the educational environment, and in some cases, their generational status and gender. The study focused on capturing the voices of these students, many of whom highlighted both challenges and benefits as a result of their experiences with support services in educational settings. They also discussed the impact of cultural and family influences growing up, highlighting the differences in acculturation and assimilation (Aguayo et al., 2011) that take place for a multigenerational Raza student population across genders. Overall, the voice of the interviewees emphasizes the need for institutions, particularly those that are identified as HSIs, to examine the meaning of the HSI label as it relates to the specific needs of the diverse Raza student population at the institution (Doran, 2015)

\section{Justification for Generational and Gender Focus}

Malcom-Piqueux and Bensimon (2015) have highlighted the need for HSIs to engage in the assessment needed for institutions to be truly Raza serving, a move that is necessary to ensure HSIs are accepting the responsibility for educating a group that will represent $31 \%$ of the U.S. population by 2060 (Santiago et al., 2015). In addition, the generational diversity of Raza in this country requires that research on this population examine whether or not generational status has an impact on the educational experience of students. Of the Raza students who answered the survey, $20 \%$ identified as first generation, $59 \%$ as second generation, and $16 \%$ as third generation. These figures vary 
from National data in 2008 that found $21 \%$ of Raza undergraduates were first generation, $45 \%$ were second generation, and $33 \%$ were $3^{\text {rd }}$ generation (Santiago et al., 2015). One reason for the low number of third generation respondents in the sample as compared to the national average could be due to some third generation Raza students who might not necessarily identify as Raza and thus didn't think the survey was relevant to them. This reality speaks to the potential difficulty in identifying the needs and challenges of groups based on generational status due to the impact a student's experience has on their ethnic, cultural, and racial identity. Although the third generation population is currently the lowest among the Raza generational subgroups, a large percentage (53\%) of second generation Raza are ages 18-29 (Taylor et al., 2013), signifying the third generation Raza population will continue to grow as the young adult $2^{\text {nd }}$ generation Raza population grows older and has children that identify as third generation Raza. Further, as a result of the discrepancy in the number of male Raza students who are enrolled in higher education as compared to their female counterparts - $59 \%$ female and $41 \%$ male (gender data by ethnicity was not available) at the institution that is the focus of this study - and Raza males having the lowest degree attainment rate of any subgroup in the United States (Cerezo et al., 2012), the gender experience must be accounted for when examining the needs and experiences of Raza students. In addition, the educational advancement of Raza males will continue to become more significant as the Raza college age population continues to increase - since 2004, the Raza college enrollment rate increased from 54 to $70 \%$ (Santiago et al., 2015). 


\section{Interpretation of Findings}

This study focused on answering the following questions: How do family influence and the life and educational experiences of a multi-generational Raza student population across genders impact students' perception of support? How does a multi-generational Raza student population across genders perceive the effectiveness of existing institutional supports at an HSI? What additional supports do multi-generational Raza students across genders identify as necessary to succeed at an HSI? The research questions are significant as they aimed to account for the diversity of the Raza student population and the potential for different support needs for students depending on their generational status and gender. In addition, the questions aimed to shed light on the needs of a diverse Raza student population at an HSI, a label that will become more prominent as educational institutions around the country continue to see an increase in the Raza Student population (U.S. Department of Education, 2011).

The following three themes came to light based on the narratives shared by the 25 interviewees: (1) family away from home; (2) authentic caring = sense of belonging; and (3) overcoming internalized oppression. As a result of the narratives shared and the three themes, I developed a conceptual model based on Yosso's (2005) Cultural Wealth Model that highlights six forms of capital that institutions can utilize to plan their approach for addressing the needs of Raza students at the institution: aspirational, familial, linguistic, social capital, navigational, and resistance. The Cultural Wealth Model (Yosso, 2005) is the center of the Raza College Student Support Model I developed due to the potential for 
addressing Raza student needs - as they relate to the study themes. It is my belief that the Cultural Wealth Model (2005) can serve as a tool to process the various experiences and capital Raza students bring with them to the institution, both negative and positive components. Once the experiences and capital are processed through the components in the model, the components that are most aligned with the student experience or capital (for example using the familial component to compliment the family capital the student brings with them), can be adopted as a mechanism to assist the students in addressing issues of sense of belonging, family, and internalized oppression that are impacting them at the institution.

The students' educational experience, home environment, and family influence which include the role of generational status - have intersected to create different level of oppression, sense of belonging, and family environment for the student at the institution. The troubling "overcoming internalized oppression" theme is rooted in the experiences of students before they arrived at the institution and for some, is a result of negative experiences at the institution. Whatever the case, it is necessary for institutional staff to identify ways to ensure the issues and challenges students bring with them are addressed by support services (i.e. narrative sharing and inventory assessment of student capital) while also reforming campus services that have led to further internalization of negative stereotypes (i.e. lack of authentic caring approach) and ways of thinking for students at the institution. 


\section{Educational Equity Implications}

Although this study did not focus on degree attainment specifically, the study significance is very much tied to the need for a better understanding of the Raza College student experience and the utilization of support systems that often have a direct connection to degree attainment rates. The study focus is aligned with Excelencia in Education's initiative on "Ensuring America's Future" which recommends the following three strategies: updating the internal and external campus community about efforts to increase Raza degree attainment; improving the engagement and collaboration focus on Raza degree attainment; and increasing knowledge of effective strategies aimed at increasing degree attainment for all students (Santiago \& Soliz, 2012). The implementation of such strategies will have a direct impact on Raza degree attainment rates that are tied to the economic needs of our $21^{\text {st }}$ century economy, which will require 2.3 million additional college educated workers by 2025 (Valliani, 2015). According to Santiago et al. (2015), Raza college enrollment will increase 27\% from 2011-2022, further highlighting the need to examine ways to ensure that Raza college completion is a focus for practitioners and policymakers. In California specifically, the state will need to award one million more university degrees in order to keep up with economic demands (Geiser \& Atkinson, 2010). In addition, the need for improving educational outcomes for Raza students is significant as 1 in 2 children in California identify as Raza and $38 \%$ of the state's population identifies as Raza, with over $80 \%$ identifying as a Raza of Mexican ancestry (Valliani, 2015). Further, with over one fourth of Mexican Americans (the Raza 
group that is the focus of this study) living in poverty and one third of that population not having health insurance (Gonzalez-Barrera \& Lopez, 2013), the degree attainment of the Raza population has implications for the economic prosperity of our country. An increase in educational attainment has been known to have an impact on Civic Participation (Dee, 2003), and with Raza driving the labor force for the foreseeable future (White House, 2013), the educational experience of Raza students will have a direct impact on the country's ability to stay competitive in the global economy.

At the institution that is the focus of this study, the educational equity implications for this study are tied to campus efforts to improve the institutional climate for all students. The campus recently released a set of key findings that highlight the challenges students of color face as a result of experiencing discrimination, not feeling a sense of belonging, and not feeling like the campus is doing enough to support them. These findings are aligned with the findings outlined in chapter 4 of this study, specifically the need for helping students overcome internalized oppression, ensuring institutional forces are not contributing to an increase in the oppression students are experiencing, and improving services, policies, and processes to increase the sense of belonging of Raza students.

When Raza students have a positive university experience, there can be a positive domino effect as students become capital sharing agents to others with degree aspirations, allowing our state and our country to make more progress towards our educational and economic objectives. As Lupe ( $\left({ }^{\text {st }} \mathrm{Gen}\right)$ said, "Being able to pass knowledge to other 
people, to people that I work with - not only do I refer to coworkers. I mean my -- the families that I work with. To me, that's the main point." Positive support experiences for Raza students at the institution can have a widespread impact on the people these students interact with. For example, Lupe sees herself as someone who has a duty to share the knowledge she has gained with people who in most cases do not have access to similar resources. Mary ( $1^{\text {st }}$ Gen $)$ also expressed a sense of gratitude for the support she has received and mentioned that the positive experiences she has had have helped shape her character and helped her realize that she wants to be a resource for other students like herself.

Other students like Anna ( $\left.2^{\text {nd }} \mathrm{Gen}\right)$ mentioned her desire to help her younger cousins, specifically mentioning that she will encourage them to "Go to college, make your statement. Don't give a crap about what other people say." While Anna has faced significant challenges throughout her time in college, she developed a "no quit" attitude as a result of the positive support systems she has had available to her. Anna is using the struggles she has faced throughout her life partnered with the positive support experiences on campus to fuel belief in her ability to have a positive impact on others.

\section{Implications for Theory, Policies, and Research}

The Raza College Support model that was developed for this study emphasizes the need for institutions to implement a Cultural Wealth Model (Yosso, 2005) to address student development areas that can lead to an increase in the degree attainment rates of 
Raza students. As of 2011 , more than $50 \%$ of Raza college students were enrolled as HSIs (U.S. Department of Education, 2011), highlighting the potential for HSIs to take the lead in exploring new approaches for addressing the educational and economic needs of our country. According to the U.S. Department of Education (2011), the U.S. government is committed to investing one billion dollars in HSIs over the next ten years with the end goal of a larger percentage of Raza students graduating with a university degree and contributing to global U.S. economic competitiveness. The investment in HSIs must be partnered with an increase in research that examines HSI best practices and the diverse student populations they serve. The need for research is directly tied to the need to update policies and practice that are outdated and do not contribute to an increase in academic outcomes for students at HSIs.

The Raza College Student Support Model builds on previous frameworks that are relevant to the issue of the Raza College Student experience, specifically the Cultural Wealth Model (Yosso, 2005) that was described earlier, the Bi-cultural Orientation Model (Torres, 2003) that measures Raza students' comfort with the majority culture and the culture of origin, a "lens of recognition" (Rodriguez, 2012) that acknowledges, recognizes, and legitimizes the Raza College students, and LatCrit theory, which provides a foundation for the aforementioned frameworks to account for the role of race, culture, class, behavior, and language in the Raza college experience (Yosso et al., 2009).

Specifically, Raza College Student Support Model demonstrates that a cultural wealth model must be adopted to address the needs of a multigenerational Raza student 
population across genders that arrive on campus with various perceptions of the educational environment based on their life and educational experiences and family influence. The Raza College Support Model also accounts for the educational experiences students encounter at the host institution, with a focus on identifying both negative and positive experiences, both of which can be addressed through adopting a cultural wealth model. The negative experiences need to be identified in order to address the oppressive mentality these experiences may contribute to and to identify practices and strategies that can serve as reform efforts. On the other hand, positive experiences must be examined and highlighted to emphasize what has worked and to encourage the institutional community to build on them.

As detailed in Chapter 4, a majority of third generation students shared more positive experiences about support both before arriving and during their time at the institution. However, the results were not monolithic and other third generation students, like Julieta and Ximena, spoke about challenges that quite similar to those identified by students in the first and second generation group. Specifically these included the challenges associated with navigating the current institution as well as previous educational and life experiences that led to insecurities about asking for help. While findings from the Pew Research Center (Taylor et al., 2013) provide evidence that first and second generation Raza have more similarities in the way they identify themselves as compared to third generation Americans, the same report found that second generation Raza have higher levels of education than either first or third generation Raza, a finding 
that emphasizes the need to look past generational status when examining contributing factors to Raza degree attainment.

The sense that generational status is not tied to perceptions of support is similar to past research findings that the level of acculturation as a result of a stronger sense of ethnic identity across Raza generational groups is more indicative of Raza college student challenges, with less acculturation typically resulting in lower educational outcomes (Guyll et al.,2010). As Phinney, Dennis, and Gutierrez (2005) noted, ethnic identity is constantly changing and is influenced by the lived experiences of students, a finding that counters the assumption that generational status outright dictates acculturation or perception of supports for multigenerational Raza college students. Further, a student's ability to develop a high level of congruence among the cultures and environments they navigate, especially when these environments create tensions within the student as a result of the disconnect, has an impact on the support-seeking attitudes of students (Padilla, 2007).

In regards to gender, the females in the study were more likely to bring up the role of family and the significance of their support and interactions. While males like Felipe $\left(2^{\text {nd }} \mathrm{Gen}\right)$ and Martin $\left(3^{\text {rd }} \mathrm{Gen}\right)$ discussed the role of family extensively, others focused more on discussing aspects of independence as they've navigated educational institutions throughout their life. The sense of independence that was representative of a majority of the males in this study was supported by claims from males expressing fear about being outed for utilizing services on campus, a fear that is connected to their desire to be seen 
as independent and presumably, able to take care of themselves. According to Dorame (2012), male Raza students encounter similar cultural, financial, educational stressors to female Raza students, but tend to process stressors differently depending on the gender expectations they have learned to follow. In addition, females across ethnic and racial groups are known to report higher levels of satisfaction and cultural congruence with support systems available to them (Bordes \& Arredondo, 2005), a finding that places the male educational attainment problem within the context of all ethnic and racial groups in our country. Further, for male students like Jesus and Emiliano, both of whom discussed barriers in asking for help as a result of cultural and gender dynamics growing up, it is necessary to ensure the limitation is acknowledged by Universities to ensure students are taking advantage of appropriate support services. As Gloria et al. (2009) found, Raza males only sought the support of those in support positions as a last resort. Emiliano and Jesus did mention the positive impact mentors have had on them, Jesus having his brother as a mentor and Emiliano having older cousins that helped him develop more effective support seeking strategies. This finding is supported by past research highlighting the effectiveness of mentors for Raza males specifically (Barajas \& Pierce, 2001).

Future educational policy pertaining to degree attainment for Raza populations should continue to engage methodologies that examine the specific needs of a diverse Raza population and to specifically examine best practices that assist with addressing Raza students' issues in the domains of sense of belonging and the internalized oppressive mentality these students have developed due to a variety of external factors. In 
CA specifically, as the Raza population continues to rise, state educational policies must be reflective of the population and must take a proactive approach to addressing gaps in educational outcomes between different students groups.

\section{Implications for Institutional Leaders}

The findings of this study highlight the complexities involved when examining the needs and challenges of a multigenerational Raza student population across genders. Considering the various implications that were explained throughout this chapter, it is crucial that institutional leaders and those with policy making power work to ensure there are intentional efforts to ensure the recommendations highlighted by this study are addressed. On the structural level, the institution must re-examine an organization that lacks the adequate academic, advising, career counseling, and financial aid necessary to help all student succeed, specifically multigenerational Raza and other marginalized populations. As part of an effort to increase the number of Raza who graduate from college, there is a need to improve the systems of academic support, professional development, and the collection, analysis, and use of data (American Association of Community Colleges, 2012). Recommendations for institutional administrators, faculty, and staff are outlined below. 


\section{Integrate Findings into Campus Diversity Plan}

As part of campus efforts to foster an inclusive campus environment that supports the success and growth of students, the institution that is the focus of this study released a Campus Climate survey in the Spring 2015 semester. In Fall 2015, the institution released a summary of findings for the Campus Climate Survey that highlighted four themes: the need for the institution to address and minimize discrimination and bias against students of color and LGBTQ student; the need for academic validation inside the classroom; improving interactions with faculty amongst students of color and LGBTQ students; and improving the sense of belonging for students of color and LGBTQ students. These four themes are similar to the three themes that were outlined in this study, specifically the sense of belonging and addressing issues of discrimination and bias to address student internalization of an oppressive mentality. The campus has plans to integrate recommendations from the Campus Climate results into the development of a Campus Diversity Plan that is tentatively scheduled to be completed in Fall 2016. Developing a comprehensive plan to respond to the needs of all students is also aligned with research calling for HSIs to analyze how institutional policies and practices may have a negative impact on students (Malcom-Piqueux \& Bensimon, 2015). It is my belief that changes will be recommended as a result of the campus climate findings, allowing the institution to develop an attainable action plan focused on improving outcomes for Raza students and ensuring the institution is living up to the HSI label. 
Campus Leadership Welcoming Discussion on Race and Ethnicity

According to Hendrickson, Lane, Harris, Dorman, and Ikenberry (2012), leadership that is committed to institutional change will require the financial and resource investment necessary to engage in conversations about the role of race and ethnicity on student performance and the need to revisit institutional policies and practices that have led to unequal outcomes. As part of efforts to develop the Campus Diversity Plan, institutional leaders should find ways to legitimize the struggles and experiences of all underrepresented students, specifically through the contextualization of their experiences (Rodriguez, 2012). In addition, educational leaders who develop a shared purpose within the school through a caring and supportive approach, understand the values, beliefs, and cultural traditions of students and surrounding communities, can more effectively engage stakeholders about the role of school in society and the importance of intellectual and character development for all students (Deal \& Peterson, 2009). Actions that are conveyed with care, respect, and appreciation can go a long way in developing a unified school and community culture (Bolman \& Gallos, 2011, p. 100). The creation of institutionalized spaces that encourage discussion on teaching, learning, and collaboration provides a great opportunity to share knowledge (Grubb et al., 2011). Leaders can contribute to collaboration by utilizing a strengths-based and appreciate inquiry approach that integrates the strengths of various individuals into the professional development process (Bergquist \& Pawlak, 2008). A unified approach to addressing educational issues 
at the institution will undoubtedly strengthen the collaboration that leads to innovation and informed decision-making (Deal \& Peterson, 2009).

\section{Implement Latinos Student Success (LSS) Inquiry Model}

The LSS model (Appendix F) was developed by Excelencia in Education and provides a tool for institutions to create a profile of Raza students in an effort to improve Latino Student Success at the institution. The model specifically focuses on examining ways to improve the success of Raza students without excluding the needs of others - the model is packaged as a way to serve the needs of all students at the institution, not only those of Raza descent, an approach that highlights the role HSIs can play in addressing the needs of all students at an institution (LSS Inquiry Model document). Specifically, the model ask for institutions to gain a better understanding of the Raza students at the institution, the activities and programs that are having a positive impact on Raza students, the disaggregation of data to identify the graduation rate of students based on ethnicity/race, the meaning of the HSI identity at this specific institution

\section{Culturally Relevant Professional Development}

For educators specifically, one of the first steps in helping multigenerational Raza students overcome the many institutional, societal and cultural barriers requires that educators examine their own biases, assumptions, and stereotypes about marginalized groups. The reflective process can contribute to the deconstruction of the overbearing 
structures that permeate in education and society (Brown, 2004). By examining their own inherent beliefs and assumptions, educators allow themselves to view students in a different light, particularly those that may be considered "under prepared", by adopting a more culturally relevant curriculum that acknowledges student cultural norms. This "recognition" can, potentially, address the impact of the "invisibility" discussed earlier. Adopting a "lens of recognition" (Rodriguez, 2012) highlights the need to acknowledge, recognize, and legitimize the presence of Raza college students. Just as educators at the primary and secondary level are encouraged to create a classroom environment where recognition is practiced, so too should this recognition continue at the college level in order to help Raza future professionals feel valued and visible.

Furthermore, Rodriguez (2012) notes the conceptual, pedagogical, and leadership value of expanding our perspectives on the role of recognition in the social, cultural, and educational lives of marginalized youth and young adults. The work of "contextualizing their experiences in a larger struggle for voice, identity, and existence" (p. 4) involves engaging "in an exercise" of "acknowledging the social, political, and economic conditions that plague marginalized communities with substandard schools" (p. 5).

While professional development can assist administrators, faculty, and staff in addressing the various areas mentioned above, those professionals who interact with students on a daily basis must have specialized training in working with marginalized students populations. Specifically, for college educators who might have extensive training in subject matter but minimal training in learning development, there needs to be 
an added emphasis on connecting both content mastery and student learning development (Darling-Hammond, 2010). Further, future educators must be taught to engage students through diversified models of oral and written language (Darling-Hammond, 2010). As future educators learn to work with a variety of marginalized student populations, it will be important for them to also have access to a knowledge sharing structure that allows for the distribution of practices and insight that can contribute to an improved education experience for multigenerational Raza Students.

It should come as no surprise that educating multigenerational Raza students involves a reciprocal professor-student relationship dependent on a humanizing pedagogy that contributes to the struggle for liberation because it is built on respect, authentic caring, and critical hope. Indeed, initiating real change in students, schools, and communities will require an education that is accompanied by a revolutionary love based on Carino (caring) and a true commitment to student learning (Duncan-Andrade \& Morrell, 2008). Educators can help Raza college students develop a positive self-image as it relates to race, gender, class and identity through a critical hope that opposes the hegemonic ideology found in schools at all level (Duncan-Andrade, 2009). These students must heal and love themselves in order to self-actualize and contribute to the counter-hegemonic process towards liberation. They need to learn to challenge the limitations they place on themselves as well as those that are constructed by educational and social institutions (Darder, 1997). For Raza college students specifically, educators must help students engage in dialogue that helps them understand how their educational 
and life experiences in the dominant culture have impacted the beliefs they have about themselves and have resulted in their continued oppression (Darder, 1997).

CCU educators would benefit from additional professional development that emphasize a critical pedagogical approach to education that is focused on three elements: organic intellectualism (Gramsci, 1971), praxis, and counter-hegemony. "Organic intellectualism" refers to the intellectual capacity that all students have based on their life experiences and the ways they view the world. By identifying the organic intellectualism of multigenerational Raza students, educators can engage students in praxis that is counter hegemonic and transformative. Building from Vygotsky's “zone of proximal development" will further help educators determine the strengths of each student in order to provide a learning environment that is aligned with student strengths and intellectual capacity (Duncan-Andrade \& Morell, 2008). Such an approach can be identified as an adaptive mode of teaching where the educator fosters an environment that adapts to students strengths, interests, and background (Darling-Hammond, 2010).

It is important to emphasize that a critical pedagogical approach must assure that multigenerational Raza college students are taught to become academically proficient within the current schooling system, allowing them to maneuver the system, language, and power tools of the dominant class (Solorzano \& Delgago-Bernal, 2001). Indeed, it is possible for these students to engage in a college education that teaches them about structural inequalities while also communicating the skills to navigate the current system 
and allowing them to maintain their cultural identity and ideals (Duncan-Andrade \& Morrell, 2008).

\section{Additional Support for Raza Students Transitioning into the University}

Institutional leaders should work to establish a system to address the various student transitional challenges that were discussed by students. Students like Samuel (2nd Gen), who almost didn't attend the institution due to not being clear on payment deadlines, as well as reservations and doubt as move-in day arrived, would benefit from an improved information sharing and clarification of payment deadlines for both registration and housing and a check-in system that has institutional staff follow up with students who are at potential risk. Such students would include those not registered for the upcoming semester, who are pending payments, and who are pending submission of final documents for financial aid and academic purposes. The institution must face the reality that there will be a constant tension between ensuring students submit documents and payments on time while also realizing that the "vision statement" students the institution is focused on serving oftentimes face difficulty in paying college-going expenses and also dealing with concerns from family about their upcoming transition that may lead to students to second guess their decision to attend the University.

In addition to financial challenges, those who are first generation college students would benefit from a support system that contacts them before they arrive at the institution to ensure they are clear about deadlines and also begin to feel a sense of 
belonging. Past studies have found that Raza students see the institution as unfavorable and discouraging due to instances where they feel they are treated worse than their counterparts (Yosso et al., 2009). New student orientation programming that is focused on addressing this barrier is recommended to provide incoming students an opportunity to hear the narratives of students who come from similar backgrounds, engage in teambuilding activities that allow students to connect with other students, and be provided key transitional information such as proper usage of email, iLearn, and best practices for communicating with professors.

It will also be necessary for institutions to develop programming and activities that assist in the transition from University to the real world. Julieta $\left(3^{\text {rd }} \mathrm{Gen}\right)$ specifically discussed the need for information and resources that focus on the many components of life after college, including financial literacy (focused on credit building, student loan repayment), professional development (resume, cover letter, and interview preparedness), and overcoming the emotional challenges associated with such a transition.

\section{Establish a Formal Commuter Student Support System}

The transitional support recommended above may be even more significant for Raza commuter students. Such students may have an even harder transition as a result of spending less time on the physical campus. Such commuter student support systems would help Raza students, like those who participated in this study, deal with identity challenges that result from navigating the home and institutional environment that are at 
times not compatible, and would also help them to develop a support network on campus or developing a sense of comfort with the university environment, despite the relatively limited time spent on campus in comparison to residential students.

Students like Evelyn (2nd Gen) and Amanda (1st Gen) described the need for a support group for commuter students or some type of directory that includes basic information about commuter students, their hometown, and major. This directory could serve as a resource for commuter students who are looking for students to carpool with or students in the same major who can serve as study buddies as well as systems of accountability and support. During New Student Orientation, it is recommended that the institution provide some programming that is specific to the needs of commuter students, for example a student panel that consists of commuter students who can share words of encouragement and tips for commuter student success.

\section{Comprehensive Mentoring Program for Raza Students}

The need for a continued focus on sense of belonging and developing a sense of family is evident as students who participated in this study discussed the need for supports that take an interest in the whole students and provide students with a positive experience that is rooted on guidance, authentic caring, and information sharing. I recommend the institution to consider expanding mentoring programs for Raza students, specifically those who may have more risk factors such as those in STEM majors, commuter students, and those who are first generation college students. Ultimately, the 
mentoring program is recommended for all students, as even 3rd gen students like Martin and Victoria spoke about the need for additional support and guidance from those in mentoring positions. Past research has tied mentoring to an increase in sense of belonging and college adjustment and an increase in self-efficacy (Gloria et al., 2009). A comprehensive mentor model would build on the collective approach that many Raza students value and would also serve as a strategy to address institutional culture issues that lead students to feel marginalized as a result of campus climate issues (Yosso et al., 2009). The institution can first work on ensuring mentoring services are marketed to appropriate populations. In addition, institutional staff should look at ways to provide mentor programs that are focused on addressing personal (well-being focus; peer to peer), academic (advising/study focus; faculty coach), and career (job shadowing).

The mentoring sessions should be designed to help students prioritize their studies, plan how to be successful, explore and prepare for professional careers, and identify and overcome barriers to student success. The mentor models should incorporate distinct coaching techniques for producing the best results for each student, which will require building strong relationships, continuously assess needs, have the capacity to advance students, build motivation, and help them develop strategies for continued success. Learning communities provide a great opportunity for engaging students through a structured peer support system that also serves a system of accountability and can integrate a mentorship model to help students develop in academic, personal, and career areas. 


\section{Improved Assessment of Raza Student Strengths and Areas of Need}

This issue of students wanting professors to guess what support they need highlights the need for students to be assessed on their strengths and weaknesses, and providing staff and faculty a profile for students that includes information on their needs, strengths, challenges, etc. Students in the various college support programs on campus have such a profile in place that allows staff to have a better understanding of students. While it may be difficult to identify the specific needs of all students transitioning into the University, resources such as the Noel-Levitz College Student Inventory (CSI) could serves as a resource to identify student "at-risk" factors early in their time at the institution.

CSI will assess academic motivation (study skills, intellectual interests, academic confidence, desire to finish school, attitude toward educators, technological competence), social motivation (self-reliance, sociability, leadership), general coping skills and support (sociability, family, emotional, opinion tolerance, career closure, sense of financial security), and receptivity to support services (academic assistance, personal counseling, social enrichment, career counseling, financial guidance), all areas that were discussed by the students who participated in this study. CSI will allow staff to understand individual and cohort needs and strengths, recognize students who may be at risk of leaving college, motivational patterns, and student receptivity to services. CSI provides information that can be used to implement interventions and referrals, and more importantly, will allow for effective and tactical use of institutional funding - the 
institution can prioritize addressing the needs of students who have high risk factors, according to CSI results (College Student Inventory, 2015).

Support staff can work to review CSI summary observations for participants with high risk factors, identifying and making note of their level of risk and receptivity. The summary observations will help staff prioritize interventions on an individual and cohort level.

\section{Re-thinking the Way Services are Marketed}

It is important to let students know that supports will impact them positively on a number of different levels including their academics, personal, career, social, etc. Study findings suggest the institution should work on finding more effective ways to help students make the connection between the support systems and their academics. Student responses highlight the need to emphasize how personal support systems can help students academically. Another component of this re-marketing/re-branding requires the institution to have more spaces for students to see the utilization of supports as part of the college experience, not just something that is available for a certain population or for those students who are going through challenges. Administrators can support this process by ensuring that sufficient funding is available for a variety of campus supports. Faculty can assist by providing students with extra credit for utilizing supports or by being fully informed of support services and encouraging students to utilize them as necessary. 
In addition, it is necessary to re-think the responses support staff provide to students who are interested in support, to ensure students are not simply providing a "no" answer when seeking support. Ideally, student will be provided with a list of additional supports if the support they are inquiring about is not appropriate for them. This will ensure that students do not develop this sense of "invisibility" that was discussed earlier and may increase feelings of exclusion. For Raza students who have reservations about seeking emotional support, the institution is encouraged to provide both students and parents' information on available resources during the orientation/transition period. The information should have a focus on breaking down stereotypes about emotional support and should help students and parents make the connection between emotional well-being and academic success.

\section{Recommendations for Future Research}

I recommend the continued examination of the intersection of ethnic identity, acculturation, assimilation and the role it plays in components of the Raza college student experience. A mix methods approach to this research would allow for the integration of additional variable such as social class, race, and grade point average. Race specifically could be a variable that is further examined within the Raza population as a color of a person's skin may possibly have a large impact on the student experience with supports and perceived supports. In addition, the examination of support should look at the experience of Raza students who are no longer in college, specifically those who dropped 
out, in an effort to gain a better understanding of challenges and desired supports by those who were not able to make it through their higher education. Examining this population may provide a better understanding of how Raza students reflect back on the supports they had available to them when they were in school and how they would approach them differently at this point in their life. Finally, I believe future research should focus on examining individual institutions to ensure the specific problems within that institution are identified and that strategies for improvement are appropriate for that institutional culture and most importantly, for that specific student population.

\section{Reflections on the Research Process}

As challenging as this process has been, I am grateful for the opportunity to contribute to a field of research that connects to my own experience as a Raza student navigating institutions of Higher Education. It.has been a humbling experience that has instilled in me a curiosity that will undoubtedly lead me to engage in future research on similar topics that have as a foundation the desire to improve the educational experience and academic outcomes for all students. 


\section{Conclusion}

With 15 California State University (CSU) campuses that have been designated as HSIs, the CSU is a leader in creating opportunities for the Raza students of California to reach their education goals. Approximately 62 percent of Raza in California with a bachelor's degree studied at the CSU. Today, 33.4\% of CSU students are Raza-the highest population among CSU students (Thara, 2014). The reality is that our country will need more than a new set of national goals to direct our nation toward the kind of learning necessary for the $21^{\text {st }}$ century. A significant component of that work will require improvements to the degree attainment rates and educational experience of multigenerational Raza students, an initiative that has a direct role in addressing the economic and social disparities in our country. I must acknowledge that there will be people who view some of the recommendations of this study as a "coddling" of our students. On the contrary, the recommendations in this study are necessary when considering the implications of a hegemonic educational system that has done very little to build students back up after breaking them down, specifically students from lowsocioeconomic backgrounds and those outside the culture of power. The students in this study do not want an easy way out by any means. They just want a fair chance to prove themselves and have someone available who is willing to meet them halfway to understand where they are coming from. This study acknowledges that simply pampering students will only reinforce a deficit mindset and strengthen an exclusionary culture that limits Raza students' ability to succeed at all educational and professional levels. 
Indeed, mentors and educators must help Raza understand the "culture of power" within which they live. Helping student's balance critical understanding with positivity and resilience is possible through support and positive reinforcement elements, providing for Razas' intellectual and personal development while also instilling in them a desire to change the systems in place, flipping the "resistance" mentality some of these students demonstrated to one that focuses on participating in the system in an effort to change it. As was evident with some of the students in this study, a deficit mindset can cause a student to lose belief in her own abilities and disengage from certain conversations and groups at the professional level. Educators and mentors must thus develop a "counterassumption" mentality in order to help rectify the many assumptions commonly held about the culture, family dynamics, and experiences of Raza students (Rodriguez, 2012).

One thing is certain - our educational systems must become more responsive to the many variables of identity found in students like Carina, Juan, Alondra, and Ximena in order to be truly transformative for the Raza population. Such a responsive approach should include a focus on transformative recognition that helps Raza visualize how their educational experiences fit into the larger context of social change and empowerment (Rodriguez, 2012). Further, there is a need for educators to help Raza students find a sense of "home" and comfort through helping them resolve the tensions and ambiguity found while living across multiple cultures. Support staff and faculty must make changes in pedagogy, rooting lessons in students' experiences and concerns, to help students from 
all cultural groups make sense of their own experiences and the challenges they are attempting to overcome (Reddick \& Saenz, 2012).

How it looks to recognize and legitimize Raza students will vary, depending on their specific family, cultural, academic, and social backgrounds, as we work towards educational systems capable of responding to students as unique individuals. Ultimately, it is hoped that this work contributes to the conversation that is working to move our society towards one that values the intellectual development, self-reflection, critical pedagogy, love, respect, and compassion necessary to address the injustices confronted in the economic, cultural, political, and educational domain (Tejeda, Espinoza \& Gutierrez, 2003). 


\section{References}

Aguayo, D., Herman, K., Ojeda, L., \& Flores, L. Y. (2011). Culture predicts Mexican Americans' college self-efficacy and college performance. Journal of Diversity in Higher Education, 4(2), 79-89.

Alon, S., Domina, T., \& Tienda, M. (2010). Stymied mobility or temporary lull? The puzzle of lagging Hispanic college degree attainment. Social Forces, 88(4), 18071832.

American Association of Community Colleges. (2012). Reclaiming the American dream: Community colleges and the nation's future. 21 st Century Commission on the Future of Community Colleges. Retrieved from: http://www.aacc21 stcenturycenter.org/

Apple, M. (1990). Ideology and curriculum (2nd ed.). New York: Routledge.

Arciniega, T. (2012). The Crucial role of Hispanic-serving institutions in the education of Latino/a youth. Journal of Latinos in Education, 11(3), 150-156.

Ayala, M.I. (2012). The state of research in Latino academic attainment. Sociological Forum, 27(4), 1037-1045.

Barajas, H., \& Pierce, J. (2001). The significance of race and gender in school success among Latinas and Latinos in college. Gender \& Society, 15(6), 859-878.

Becerra, D. (2010). Difference in perceptions of barriers to college enrollment and the completion of a degree among Latinos in the United States. Journal of Hispanic Higher Education, 9(2), 187-201. 
Bergquist, W.H., \& Pawlak, K. (2008). Engaging the six cultures of the academy. San Francisco, CA: John Wiley \& Sons.

Bolman, L. G., \& Gallos, J. V. (2011). Reframing academic leadership. San Francisco, CA: Jossey-Bass.

Bordes, V., \& Arredondo, P. (2005). Mentoring and 1st-year Latina/o college students. Journal of Hispanic Higher Education, 4(2), 114-133. Retrieved from http://jhh.sagepub.com/content/4/2/114.full.pdf

Bordes-Edgar, V., Arredondo, P., Kurpius, S.R., \& Rund, J. (2011). A longitudinal analysis of Latina/o students' academic persistence. Journal of Hispanic Higher Education, 10(4), 358-368.

Brown, K.M. (2004). Leadership for social justice and equity: Weaving a transformative framework and pedagogy. Educational Administrative Quarterly, 40(1), 79-110.

Buchmann, C., \& DiPrete, T. A. (2006). Growing female advantage in college completion: The role of family background and academic achievement. American Sociological Review, 71(4), 515-541.

Calabrese Barton, A., \& Yang, K. (2000). The culture of power in science education: Learning from Miguel. Journal of Research in Science Teaching, 37(8), 871-889. Castillo, L. G., Conoley, C. W., Choi-Pearson, C., Archuleta, D. J., Phoummarath, M. J., \& Landingham, A. V. (2006). University environment as a mediator of Latino ethnic identity and persistence attitudes. Journal of Counseling Psychology, 53(2), 267-271. 
Cerezo, A., Lyda, J., Beristianos, M., Enriquez, A., \& Connor, M. (2012). Latino men in college: Giving voice to their struggles and triumphs. Psychology of Men \& Masculinity, 1-16

Chapman, T. K. (2005a). Expressions of "voice" in portraiture. Qualitative Inquiry, 11, $27-51$.

College Student Inventory. (2015). Retrieved December 15, 2015, from https://www.ruffalonl.com/college-student-retention/retention-managementsystem-plus/college-student-inventory

Creswell, J. W. (2009). Research design: Qualitative, quantitative, and mixed methods approaches (3rd ed.). Los Angeles, CA: Sage.

Cuellar, M. (2012). Latino student success in higher education: Models of empowerment at Hispanic-Serving Institutions (HSIs), emerging-HSIs, and non-HSIs. Retrieved from Proquest Digital Dissertations (AAT: 3497388)

Dance, J., Gutierrez, R. and Hermes, M. (2010). More like jazz than classical: Reciprocal Interactions among educational researchers and respondents. Harvard Educational Review, 80(3), 327-351

Darder, A. (1997). Creating the conditions for cultural democracy in the classroom. In A. Darder, R.D. Torres, \& H. Gutierrez (Eds.), Latinos in education (pp.331350). New York: Routledge

Darling-Hammond, L. (2010). The flat world and education. New York, NY: Teachers College Press. 
Deal, T.E. \& Peterson, K.D. (2009). Shaping school culture. San Francisco, CA: JosseyBass.

Dee, T. (2003). Are there civic returns to education? Journal of Public Economics, 88, 1697-1720.

De Leon, S.A. (2005). Assimilation and ambiguous experience of the resilient male Mexican immigrants that successfully navigate American higher education. Ed.D Dissertation, University of Texas at Austin.

DiPrete, T. A., \& Buchmann, C. (2006). Gender-specific trends in the value of education and the emerging gender gap in college completion. Demography, 43(1), 1-24.

Dorame, F. (2012). Latino males' degree aspirations in community college: A qualitative study. Ed.D dissertation, School of Education, California Lutheran University.

Doran, E. (2015). Negotiating access and tier one aspirations: The historical evolution of a striving Hispanic-Serving Institution. Journal of Hispanic Higher Education, 112.

Douglass, J. A. (2010). From chaos to order and back? A revisionist reflection on the California Master Plan for Higher Education@50 and thoughts about its future (No. CSHE.7.10). Research \& Occasional Paper Series. University of California, Berkeley, Center for Higher Studies in Education.

Duncan-Andrade, J. \& Morrell, E. (2008). The art of critical pedagogy. New York: Peter Lang.

Duncan-Andrade, J. M. R. (2009). Note to educators: Hope required when growing roses 
in concrete. Harvard Educational Review, 79(2), 181-194.

Figueroa, J. L. (2002). Out of the neighborhood and into the ivory tower. Understanding the schooling experiences of Latino male undergraduate attending an institution of higher education. Unpublished doctoral dissertation, University of California, Berkeley.

Fontana, A. \& Frey, H. (2008). The interview: From neutral stance to political involvement, In N.K. Denzin \& Y.S. Lincoln (Eds.), Collecting and interpreting qualitative materials. London: Sage.

Galdeano, E., Flores, A., \& Moder, J. (2012). The Hispanic association of colleges and universities and Hispanic-serving institutions: Partners in the advancement of Hispanic higher education. Journal of Latinos in Education, 11(3), 157-162.

Gee, J. P. (2004). Discourse analysis: What makes it critical? In R. Rogers (Ed.), An introduction to critical discourse analysis in education (pp. 19-50). Mahwah, New Jersey: Lawrence Erlbaum Associates.

Geiser, S. \& Atkinson, R.C. (2010). Beyond the Master Plan: The case for restructuring baccalaureate education in California (No. CSHE.16.10). Research \& Occasional Paper Series. University of California, Berkeley, Center for Higher Studies in Education.

Glaser, B. G., \& Strauss, A. L. (1967). Discovery of grounded theory: Strategies for qualitative research. Chicago: Aldine. 
Gloria, A. M., Castellanos, J., Scull, N. C., \& Villegas, F. J. (2009). Psychological coping and well-being of male Latino undergraduates: Sobreviviendo la Universidad. Hispanic Journal of Behavioral Sciences, 31(3), 317-339

Gonzalez-Barrera, A., \& Lopez, M. (2013). A demographic portrait of Mexican-origin Hispanics in the United States. Retrieved February 5, 2015, from http://www.pewhispanic.org/files/2013/05/2013-04_Demographic-Portrait-ofMexicans- in-the-US.pdf

Goya, G., Vance, E., \& Serros, L. (2014). The right to dream: Promising practices improve odds for Latino men and boys. Retrieved December 13, 2015, from https://www.hiponline.org/storage/documents/HIP-MENANDBOYS-THE$\underline{\text { RIGHT-TO-DREAM.pdf }}$

Gramsci, Antonio (1971). Selections from the prison notebooks of Antonio Gramsci, New York: International Publishers.

Greene, D., \& Oesterreich, H. (2015). White profs at Hispanic-serving institutions: Radical revolutionaries or complicit colonists? Journal of Latinos in Education, 11(3), 168-174.

Grubb, N., Boner, E., Frankel, K., Parker, L., Patterson, D., Gabrier, R., Hope, L., Schiorring, E., Smith, B., Taylor, R., Walton, I., Wilson, S. (2011, December). Other institutional effects on instruction and innovation (Working Paper 9).

Gutierrez, R. (2009). Hispanic identities in the southwestern United States. In Katzew I. \& Taylor W., (Eds.), Race and classification: The case of Mexican Americans. 
Stanford, CA: Stanford University Press.

Guiffrida, D. A. (2006). African American student organizations as agents of social integration. Unpublished manuscript, University of Rochester, Rochester, NY.

Guyll, M., Madon, S., Prieto, L., \& Scherr, K.C. (2010). The potential roles of selffulfilling prophecies, stigma consciousness, and stereotype threat in linking Latino/a ethnicity and educational outcomes. Journal of Social Issues, 66(1), 113130.

Hendrickson, R. M., Lane, J. E., Harris, J. T., Dorman, R. H., \& Ikenberry, S. (2012). Academic leadership and governance of higher education: A guide for trustees, leaders, and aspiring leaders of two- and four-year institutions. Sterling, VA: Stylus Publishing.

Hurtado, S., \& Ruiz, A. (2012). Realizing the potential of Hispanic-serving institutions: Multiple dimensions of institutional diversity for advancing Hispanic higher education. Hispanic Association of Colleges and Universities. Retrieved from http://www.hacu.net/images/hacu/OPAI/H3ERC/2012 papers/Hurtado\%20ruiz\% 20-\%20realizing\%20the $\% 20$ potential\%20of $\% 20 \mathrm{hsis} \% 20$ \%20updated\%202012.pdf

Keels, M. (2013). Getting them enrolled is only half the battle: College success as a function of race or ethnicity, gender, and class. American Journal of Orthopsychiatry, 83(2), 310-322.

Kim, S. Y., \& Chao, R. K. (2009). Heritage language fluency, ethnic identity, and school 
Effort of immigrant Chinese and Mexican adolescents. Cultural Diversity \& Ethnic Minority Psychology, 15, 27-37.

Kuh. G. D., and Love, P.G. (2000). A cultural perspective on student departure. In J.M. Braxton (Ed.), Reworking the student departure puzzle (pp. 96-212). Nashville, TN: Vanderbilt University Press.

Ladson-Billings, G. (2000). Racialized discourses and ethnic epistemologies. In N. K. Denzin \& Y.S. Lincoln (Eds.), Handbook of qualitative research (Vol. 2), pp. 257-278). Thousand Oaks, CA: Sage

Lee, J. L., \& Ransom, T. (2011). The educational experience of young men of color: A review of research, pathways and progress. Retrieved from College Board Advocacy \& Policy Center website: http://advocacy.collegeboard.org/sites/default/files/EEYMCResearchReport 0.pdf.

Lee Jr., J., \& Keys, S.W. (2013). APLU: Committed to Latino student success. Issue Brief, Report No. 3000-IB1. Retrieved December 3, 2015, from http://www.aplu.org/library/aplu-committed-to-latino-student-success-andsupporting-hispanic-serving-institutions/file

Lugg, C. A. (2003). Sissies, faggots, lezzies, and dykes: Gender, sexual orientation, and the new politics of education? Educational Administration Quarterly, 39(1), 95134. 
Malcom, L.E., Bensimon, E.M., \& Davila, B. (2010, November). (Re)Constructing Hispanic-Serving Institutions: Moving beyond numbers towards student success. Ames, IA: Iowa State University.

Malcom-Piqueux, L., \& Bensimon, E. (2015). Design principles for equity and excellence at Hispanic-serving institutions. Perspectivas: Issues in Higher Education Policy and Practice, (4). Retrieved December 13, 2015, from http://cue.usc.edu/tools/publications/design principles for equity and excellenc e_at_hispanic-serving_institutions.html

Mendoza, O.A. (2012). Exploring the Chicano/Latino male narrative of higher education attainment via community colleges. Master's thesis, Department of Educational Leadership and Policy Studies, California State University, Sacramento.

Nunez, A.M., Sparks, P.J., \& Hernandez, E.A. (2011). Latino access to community colleges and Hispanic-Serving Institutions: A national study. Journal of Hispanic Higher Education, 10(1), 18-40

Obama, B. (2009, February). Remarks of President Barack Obama-As prepared for delivery. Address to Joint Session of Congress, Washington, D.C.

Ojeda, L., Flores, L., \& Navarro, R. (2010). Social cognitive predictors of Mexican American college students' academic and life satisfaction. Journal of Counseling Pscyhology, 58(1), 61-71.

Ojeda, L., Navarro, R. L., \& Morales, A. (2011). The role of la familia on Mexican 
American men's college persistence intentions. Psychology of Men \& Masculinity, 12(3), 216-229.

O'Meara, D.J. (2012). Mexican American first-generation/low-income students: a rural community college, TRiO student support services experience. Ed.D dissertation, School of Education, Drexel University.

Oseguera, L., Locks, A., \& Vega, I. (2009). Increasing Latina/o students' baccalaureate attainment: A focus on retention. Journal of Hispanic Higher Education, 8(1), 2353.

Owens, J., Lynch, S.M. (2012). Black and Hispanic immigrants' resilience against negative-ability racial stereotypes at selective colleges and universities in the United States. Sociology of Education, 85(4), 303-325.

Padilla, R. (2007). Camino a la Universidad: The road to college. Indianapolis, IN: Lumina Foundation for Education.

Patton, M. Q. (1990). Qualitative evaluation and research methods (2nd ed.). Newbury Park, CA: Sage Publications.

Phinney, J., Dennis, J. M., \& Gutierrez, D. (2005). College orientation profiles of Latino students from low-socioeconomic backgrounds: A cluster analytic approach. Journal of Hispanic Behavioral Sciences, 27(4), 1-22.

Portes, A., \& Rumbaut, R. G. (2001). Legacies: The story of the immigrant second Generation (pp.233-268). Berkeley: University of California Press.

Reason, R. D. (2009). An examination of persistence research through the lens of a 
comprehensive conceptual framework. Journal of College Student Development, $50(6), 659-682$.

Reddick, R.J. \& Saenz, V.B. (2012). Coming home: Hermanos académicos reflect on paths and present realities as professors at their alma maters. Harvard Educational Review, 82(3), 353-380.

Rendón, L. I. (2009). Sentipensante (sensing/thinking) pedagogy: Educating for wholeness, social justice and liberation. Sterling, Virginia: Stylus.

Rendón, L. I., \& Muñoz, Susana M. (2011). Revisiting validation theory: Theoretical foundations, applications and extensions. Enrollment Management Journal, 5(2), 12-33. Retrieved from http://www.tgslc.org/pdf/emj-s11-validation.pdf Rodríguez, L. F. (2012). Everybody grieves, but still nobody sees: Toward a theory of recognition for students of color in U.S. education. Teachers College Record, $114,1-31$.

Rumbaut, R. (2009) Pigments of our imagination: On the racialization and racial identity of "Hispanics" and "Latinos." In Cobas, J., Duany, J. \& Feagin, J. How the United States racializes Latinos: White hegemony \& its consequences (pp.1536). Boulder: Paradigm Publishers.

Saenz, V. B., \& Ponjuan, L. (2009). The vanishing Latino male in higher education. Journal of Hispanic Higher Education, 8(1), 54-89. 
Santiago, D., Galdeano, E.C., \& Taylor, M. (2015). The condition of Latinos in education: 2015 factbook. Retrieved December 13, 2015, from http://www.edexcelencia.org/research/2015-factbook

Santiago, D., \& Soliz, M. (2012). Ensuring America's future by increasing Latino college completion. Retrieved December 13, 2015, from http://www.edexcelencia.org/ensuring-americas-future

Saldaña, J. (2012). The coding manual for qualitative researchers (2nd ed.). Thousand Oaks, CA: Sage.

Schwartz, J.L., Donovan, J., \& Guido-DiBrito, F. (2009). Stories of social class: Selfidentified Mexican male college students crack the silence. Journal of College Student Development, 50(1), 50-66.

Silverman, D. (2010). Doing qualitative research (3rd ed.). Thousand Oaks, CA: Sage Publications.

Solorzano, D. and Delgado-Bernal, D. (2001). Examining transformational resistance through a critical race and LatCrit theory framework. Urban Education, 36(3), 308-342.

Solorzano, D. G., Villalpondo, O., \& Oseguera, L. (2005). Educational inequities and Latina/o undergraduate students in the United States: A critical race analysis of their educational progress. Journal of Hispanic Higher Education, 4(3), 272-294. Retrieved from http://jhh.sagepub.com/content/4/3/272.full.pdf 
Strayhorn, T. L. (2010). When race and gender collide: Social and cultural capital's influence on the academic achievement of African American and Latino males. The Review of Higher Education, 33(3), 307-332.

Taylor, P., et al. (2013). Second-generation Americans: A portrait of the adult children of immigrants. Retrieved February 5, 2015, from http://www.pewsocialtrends.org/files/2013/02/FINAL_immigrant_generations_re port_2-7-13.pdf

Tejeda, C. Espinoza, M. \& Gutierrez, K. (2003). Toward a decolonizing pedagogy: Social justice reconsidered. In P.P Trifonas, (Ed). Pedagogies of difference: Rethinking Education for Social Change. (pp. 10-40). New York: Routledge.

Thara, S. (2014, October 27). CSU Presidents Champion Hispanic Success in Higher Education. Retrieved February 16, 2015, from http://blogs.calstate.edu/pa/news/?p=5237

Torres, V. (2003). Influences on ethnic identity development of Latino college students in The first two years of college. Journal of College Student Development. 44(4), $532-547$.

Valenzuela, A. (1999). Subtractive schooling: U.S. Mexican youth and the politics of caring. Albany: State University of New York Press.

U.S. Department of Education. (2011). Winning the future: Improving education for the Latino community. Washington, DC: U.S. Government Printing Office. 
Retrieved from

https://www.whitehouse.gov/sites/default/files/rss_viewer/WinningTheFutureImp rovingLatinoEducation.pdf

U.S. Department of Education. (2015). Hispanics and STEM education. Washington DC: U.S. Government Printing Office. Retrieved from http://www2.ed.gov/about/inits/list/hispanic-initiative/stem-factsheet.pdf

White House (2013). An America built to last: White House Hispanic community action summits - final report. Washington DC: U.S: Government Printing Office.

Retrieved from

https://www.whitehouse.gov/sites/default/files/wh_hispanic_action_summits_rep ort.pdf

Wood, L. A., \& Kruger, R.O. (2000). Doing discourse analysis: Methods for studying action in talk and text. Thousand Oaks, CA: Sage.

U.S. Census Bureau. (November 19, 2012). American Community Survey, 2011 American Community Survey 1-Year Estimates, Table DP05; generated by Omar Murillo; using American FactFinder; <http://factfinder2.census.gov>

Valliani, N. (2015). The state of higher education in California. Retrieved October 8, 2015, from http://collegecampaign.org/wp-content/uploads/2015/04/2015-State-of-HigherEducation_Latinos.pdf

Yosso, T. (2005). Whose culture has capital? A critical race theory discussion of 
Community cultural wealth. Race Ethnicity and Education, 8(1), 69-91.

Yosso, T.J., W.A. Smith, M. Ceja, \& D.G. Solórzano. (2009, Winter). Critical race theory, racial microaggressions, and campus racial climate for Latina/o undergraduates. Harvard Educational Review, 79(4), 659-690.

Zavala, M. (2013). What do we mean by decolonizing research strategies? Lessons from decolonizing, Indigenous research projects in New Zealand and Latin America. Decolonization: Indigeneity, Education \& Society, 2(1), 55-71.

Zepke, N., Leach, L. (2005). Integration and adaptation: Approaches to the student retention and achievement puzzle. Active Learning in Higher Education, 6(1), 4659. 


\section{Appendix A \\ Interview Questions}

1. Tell me about a time when you felt well supported?

2. More broadly then, what does support mean to you?

3. What types of support services or support systems do you utilize on campus that you believe have had a positive impact your well-being and academic success?

4. What types of additional supports do you utilize outside of the traditional campus setting in an effort to impact your well-being and academic success?

5. What are some supports that are available to you that you don't take advantage of? Why not?

6. What is the determining factor in taking/not taking advantage of the support systems available to you?

7. What additional supports would you like to have available that you believe will have a positive impact on your well-being and academic success?

8. Do past life and/or academic experiences have an impact on the supports that you do/do not utilize?

9. How have your experiences with support impacted other areas of your life outside of academics?

10. Do you see a difference between academic, personal, and social support services/systems? Why or why not?

11. Currently, which type of support system do you take most advantage of and why?

12. Are there any supports that you would like to take advantage of but do not due to a past negative experience that you associate with that support system/service?

13. What are some traits of people you want to see in a supportive environment? 

14. Can you speak about your experiences utilizing support as a child and teen
(pre-college)? 


\section{Appendix B \\ Recruitment Email}

Subject: Dissertation Survey - Best Practices for Supporting Hispanic Students at CCU (pseudonym used throughout appendices).

\section{PLEASE COMPLETE THE SURVEY HERE}

Data collected from this survey will be used for completion of a doctorate degree in Educational Leadership at San Francisco State University. The purpose of this research is to identify best practices for supporting Hispanic students across generations and gender at CSUMB, a 4-year Hispanic Serving Institution (HSI).

The researcher, Omar Murillo, is a graduate student at San Francisco State University conducting research for an Educational Doctorate degree (Ed.D) in the SFSU Department of Education. You are being asked to participate in this study because you are a student 18 years or older that is identified as "Hispanic" in the CCU Student Database.

If you complete the survey, you may be one of 20 students invited to participate in a face to face interview with the researcher. The interview will take approximately 30-45 minutes. Note: completing the survey does not require you to then participate in the interview. Once the survey data is analyzed, the researcher will contact 20 of the students who completed the initial survey in order to request an interview.

The survey should take approximately 5 minutes to complete. Any questions or concerns should be directed to the principal investigator, Omar Murillo, at omurillo@csumb.edu or the research advisor, Professor Barbara Henderson, at barbarah@sfsu.edu.

Respectfully,

Omar Murillo 


\section{Appendix C}

\section{Seven-Question Survey}

1. Full Name

2. Gender

a. Male

b. Female

c. Other:

3. Please identify yourself as one of the following:

a. $1^{\text {st }}$ Generation (born in Mexico/Central American Country)

b. $2^{\text {nd }}$ Generation (born in US; one or more parents/guardian born in U.S.)

c. $3^{\text {rd }}$ Generation (born in US; one or more parents born in US; one or more grandparents born in the U.S.)

d. Born in a country other than Mexico, Central American Countries, or US. Please name the country:

4. Hometown (include state if outside of CA):

5. College Major:

6. How do you identify yourself? Please choose one

a. Chicano

b. Mexican-American

c. Hispanic

d. Latino

e. Other:

7. Please list any CSUMB clubs, support programs, sports, and/or organizations that you are affiliated with (leave blank if no affiliation): 


\section{Appendix D \\ Email sent to students who completed the survey}

Subject: [Next Steps] Dissertation Survey - Best Practices for Supporting Hispanic Students at CCU

Thank you for completing the survey that will assist in the data collection phase of my dissertation research. You have been identified as a potential candidate for the "2nd generation female" group that will be interviewed for this research study. If you are willing to participate in an interview, please reply to this message with the following information:

- Your Class Standing for the Fall 2015 semester (Incoming Freshman, Incoming Transfer, 2nd year, 3rd year, 4th year, 5th year, etc.):

- Your current location and availability (days/times) for an in-person interview from July 24th - August 21 st (I am located in Marina, CA). If you are not available in-person until the beginning of the semester, please let me know:

- Your telephone \# (cell phone preferred) and the best day/time to reach you:

Additional information about the interview component of this research is below:

You may be one of 20 students invited to participate in a face to face interview with the researcher. The interview will take approximately 30-45 minutes. Note: completing the survey does not require you to then participate in the interview.

The interview will be audio recorded to ensure accuracy in reporting your statements. The interview will take place at a time/place that is convenient for you. The researcher may contact you later to clarify your interview answers for approximately fifteen minutes within three months of the interview. Total time commitment will be 5 minutes (if only completing the survey) or 80 minutes (if participating in the survey, interview, and postinterview discussion session).

Please do not hesitate to contact me if you have any questions.

Thank you, 
Omar Murillo

Appendix E

Interview Consent Form (for students to sign before the interview)

San Francisco State University

Informed Consent to Participate in Research

Generations Apart: Effective Support Systems for Multi-Generational Hispanic

Students at an HSI

\section{A. PURPOSE AND BACKGROUND}

The purpose of this research is to identify best practices for supporting Hispanic students across generations and gender at a 4-year Hispanic Serving Institution (HSI).

The researcher, Omar Murillo, is a graduate student at San Francisco State University conducting research for an Educational Doctorate degree (Ed.D) in the SFSU Department of Education. You are being asked to participate in this study because you are a student that is identified as "Hispanic" in the CCU Student Database or because you were referred to the researcher.

\section{B. PROCEDURES}

If you agree to participate in this research, the following will occur:

- you will be sent an email invitation to complete a short 5-7 question survey that will take approximately 5 minutes to complete.

- you may then be one of 30 students invited to participate in a face to face interview with the researcher. The interview will take approximately 30 45 minutes. Note: completing the survey does not require you to then participate in the interview. Once the survey data is analyzed, the researcher will contact 30 of the students who completed the initial survey in order to request an interview.

- the interview will be audio recorded to ensure accuracy in reporting your statements.

- the interview will take place at a time/place that is convenient for you.

- the researcher may contact you later to clarify your interview answers for approximately fifteen minutes within three month of the interview. 
- total time commitment will be 5 minutes (if only completing the survey) or 80 minutes (if participating in the survey and interview).

\section{RISKS}

There is a risk of loss of privacy. However, no names or identities will be used in any published reports of the research. Only the researcher will have access to the research data. There is a risk of discomfort or anxiety due to the nature of the questions asked; however, the participant can answer only those questions he/she chooses to answer, and can stop participation in the research at any time.

\section{CONFIDENTIALITY}

The research data will be kept in a secure location and only the researcher will have access to the data. In order to minimize the risk, the researcher will store electronic files in password protected folders and will save physical documents in locked cabinets. Because the data could be used to develop a follow-up study, all data will be kept for three years. Audio tapes will not be destroyed initially because the tapes may include linguistic/behavioral data that could be analyzed in the future. After three years, original audio recordings and transcripts will be destroyed.

\section{E. DIRECT BENEFITS}

The students may experience an indirect benefit from helping university leaders understand the particular characteristics and experiences of multi-generational Hispanic students

\section{F. COSTS}

There will be no cost to you for participating in this research.

\section{G. COMPENSATION}

There will be no compensation for participating in this research.

\section{H. ALTERNATIVES}

The alternative is not to participate in the research.

\section{OUESTIONS}

You have spoken with Omar Murillo about this study and have had your questions answered. If you have any further questions about the study, you may contact the researcher by email at omurillo@csumb.edu or you may contact the researcher's advisor, Professor Barbara Henderson at barbarah@sfsu.edu 
Questions about your rights as a study participant, or comments or complaints about the study, may also be addressed to the Human and Animal Protections at 415: 338-1093 or protocol@sfsu.edu.

\section{J. CONSENT}

You have been given a copy of this consent form to keep.

PARTICIPATION IN THIS RESEARCH IS VOLUNTARY. You are free to decline to participate in this research, or to withdraw your participation at any point, without penalty. Your decision whether or not to participate in this research will have no influence on your present or future status at San Francisco State University.

Signature

Date:

Research Participant

Signature

Date:

Researcher 
Appendix F

\section{Latino Student Success (LSS) Inquiry Model}

Excelencia in Education's LSS Inquiry Model provides an approach for developing an institutional profile of Latino students while also building campus support to address the critical educational challenge and public policy imperative - accelerating Latino student success in higher education.

The Model is a work in progress and is being developed in collaboration with and feedback from numerous community colleges and colleges/universities. Visit www.EdExcelencia.org for updates as well as examples and strategies to accelerate Latino student success.

\section{Why use a Latino Student Success Inquiry Model?}

- To focus on Latino students and identify opportunities to improve their success; this is not to the exclusion of other students, but starting with Latinos, as opposed to assuming all students are engaged and served equally well. This creates the opportunity for an institution to "see" issues in their institution in a new way with a Latino lens.

- To create a variety of quantitative and qualitative indicators to better understand institutional support and Latino student progress.

- To engage leaders at multiple institutional levels and facilitate informed discussions on how to be more aware and inclusive of Latino students

The LSS Inquiry Model includes the following three components that follow:

Getting Started: setting up an organizational team to understand and address Latino student success 
Guiding Questions: These questions are intended to begin the examination of institutional or organizational strategies and resources available to address Latino student success

Mining Your Institution's Data: This template is intended to guide collection of key data for better understanding your current student profile.

\section{GETTING STARTED}

1. Presidential support and administrative engagement are important. With these in place, create a campus team with reach and expertise that includes leadership in:

- Academic Affairs

- Student Affairs

- Institutional Research

2. Assemble the information to explore:

- What do you know about Latino students at your institution and in your community?

- What can you do to improve Latino student success?

3. Answer the following Guiding Questions to frame your inquiry

\section{GUIDING OUESTIONS}

1. How would you define student success at your institution?

- How would you describe Latino student success?

- What is your institution doing that is having a positive effect on Latino student success? (programs, activities, investments, etc) 
- What do you think your institution could be doing better to serve Latino students?

2. What data does your institution use to inform your institutional practices? Is any of this data currently disaggregated by ethnicity? For Latinos?

3. Do you know what your graduation rate is for all students? For Latinos?

4. Do you know what your transfer rate is for all students? For Latinos?

5. What are some useful and appropriate indicators of institutional effectiveness in educating students?

- What measures of accountability are appropriate for assessing institutional effectiveness in educating Latino students?

6. What role does faculty play, if any, in Latino student success?

7. If you could only highlight one thing that you are doing at your campus to improve Latino student success, what would it be?

8. How does your campus academically serve Latino students?

9. Does your campus define itself as a Hispanic-Serving Institution (HSI)? What does it mean to you to be an HSI? * 
10. What is the goal of your institution's Title V- Developing HispanicServing Institutions grant from the U.S. Department of Education? How do you expect your Title $\mathrm{V}$ program to affect Latino student outcomes? *

*Using the federal definition

** Pertinent for current Title $\mathrm{V}$ recipients

\section{MINING YOUR INSTITUTION'S DATA}

The following data profile helps campus teams look at their institution's data and performance in serving Latino students.

Overview: Synthesize mission statement, current student population, goals and objectives for serving Latino students as relevant to your institution.

\section{Institutional Background}

Summary overview:

\section{Institution type:}




\begin{tabular}{|l|c|c|c|c|}
\hline Fall (academic year) & All & Latino & $\begin{array}{c}\% \\
\text { Latino }\end{array}$ & Source \\
\hline Total students & Students & Students & & IPED \\
& & & & S \\
\hline Total undergraduate students & & & & IPED \\
& & & & S \\
\hline
\end{tabular}

\begin{tabular}{|c|c|c|c|}
\hline \multicolumn{3}{|l|}{ Finance } & \multirow{3}{*}{$\begin{array}{c}\text { Source } \\
\text { IPEDS } \\
\text { Institution }\end{array}$} \\
\hline Instructional exp & per student (FTE) & $\$$ & \\
\hline Tuition and fees & Full-time $(12 \mathrm{CH}): \$$ & Per credit: \$ & \\
\hline \multicolumn{2}{|c|}{ Total research expenditures } & $\$$ & Institution \\
\hline
\end{tabular}

\section{Undergraduate Students}

Summary overview:

\begin{tabular}{|l|l|l|l|}
\hline \multicolumn{1}{|c|}{ Undergraduate Characteristics } & \multicolumn{1}{c|}{ All } & Source \\
\hline a. Students who are of traditional age (18-24) & Students & Latino & \\
\hline b. Women & & & IPEDS \\
\hline c. First-time freshman & & & IPEDS \\
\hline d. First-time, full-time freshmen & & & \\
\hline
\end{tabular}




\begin{tabular}{|l|l|l|l|}
\hline $\begin{array}{l}\text { e. Students who transfer in as new students as a } \\
\text { percent of all students }\end{array}$ & & & Institution \\
\hline f. Students enrolled full-time & & & IPEDS \\
\hline g. Students receiving financial aid & & & Institution \\
\hline h. New students taking remediation in first semester & & & Institution \\
\hline i. Students working while enrolled & & & Institution \\
\hline
\end{tabular}

\section{Faculty and Staff}

\section{Summary overview:}

\begin{tabular}{|l|c|c|}
\hline Faculty/Student Ratios & Ratio & Source \\
\hline Total Faculty/Student ratio & & IPED \\
\hline Total Latino Faculty/Latino student ratio & & Institution \\
\hline
\end{tabular}

\begin{tabular}{|l|l|l|l|l|}
\hline \multicolumn{1}{|c|}{ Faculty \& Staff Representation } & \# Latino & \# all & \% Latino & Source \\
\hline Academic Faculty (full \& part-time) & & & & Institution \\
\hline $\begin{array}{l}\text { Administrators-Executives- } \\
\text { Managers }\end{array}$ & & & & \\
\hline Professional & & & & Institution \\
\hline Secretarial-Clerical & & & & Institution \\
\hline
\end{tabular}


Service-Maintenance Institution

\section{Academic Programs}

Summary overview:

\begin{tabular}{|l|l|l|}
\hline \multicolumn{2}{|c|}{ Majors with Largest Enrollments } \\
\hline All Students & Latino Students & Source \\
\hline & & Institution \\
\hline & & Institution \\
\hline & & Institution \\
\hline & & Institution \\
\hline
\end{tabular}

\section{Academic Support Programs and Student Services}

Overview and summary of 2-3 support program highlights

\section{Developing HSI (Title V) Activities (If relevant)}

Summary of Title $\mathrm{V}$ activities and expected outcomes: 
Program activities:

Program expected outcomes

\section{Persistence and Graduation}

Summary overview:

\begin{tabular}{|l|l|l|c|}
\hline Persistence \& Transfer & All Students & Latino Students & Source \\
\hline $\begin{array}{l}\text { One-year persistence rate for first- } \\
\text { time full-time freshmen - (insert } \\
\text { academic year Cohort) }\end{array}$ & & & \\
\hline Transfer rate? & & & IPE \\
\hline $\begin{array}{l}\text { Transfer rate to another institution } \\
\text { PRIOR to completion of degree - } \\
\text { (insert academic year Cohort) }\end{array}$ & & & Institution \\
\hline
\end{tabular}

\begin{tabular}{|l|l|l|l|}
\hline Graduation & All Students & Latino Students & Source \\
\hline
\end{tabular}




\begin{tabular}{|l|l|l|l|}
\hline Total degrees awarded: Number & & & \\
(percent of total) & $(100 \%)$ & IPEDS \\
\hline $\begin{array}{l}\text { Three or six-year graduation rate for } \\
\text { first-time full-time freshmen - (insert } \\
\text { academic year Cohort) }\end{array}$ & & & \\
\hline
\end{tabular}

Students who took remediation \&

graduated during (academic year)

Institution 